\title{
A New Mechanical Characterization Method for Thin Film Microactuators and Its Application to NiTiCu Shape Memory Alloy
}

Kirk P. Seward

(Master of Science in Mechanical Engineering Thesis)

June 1999

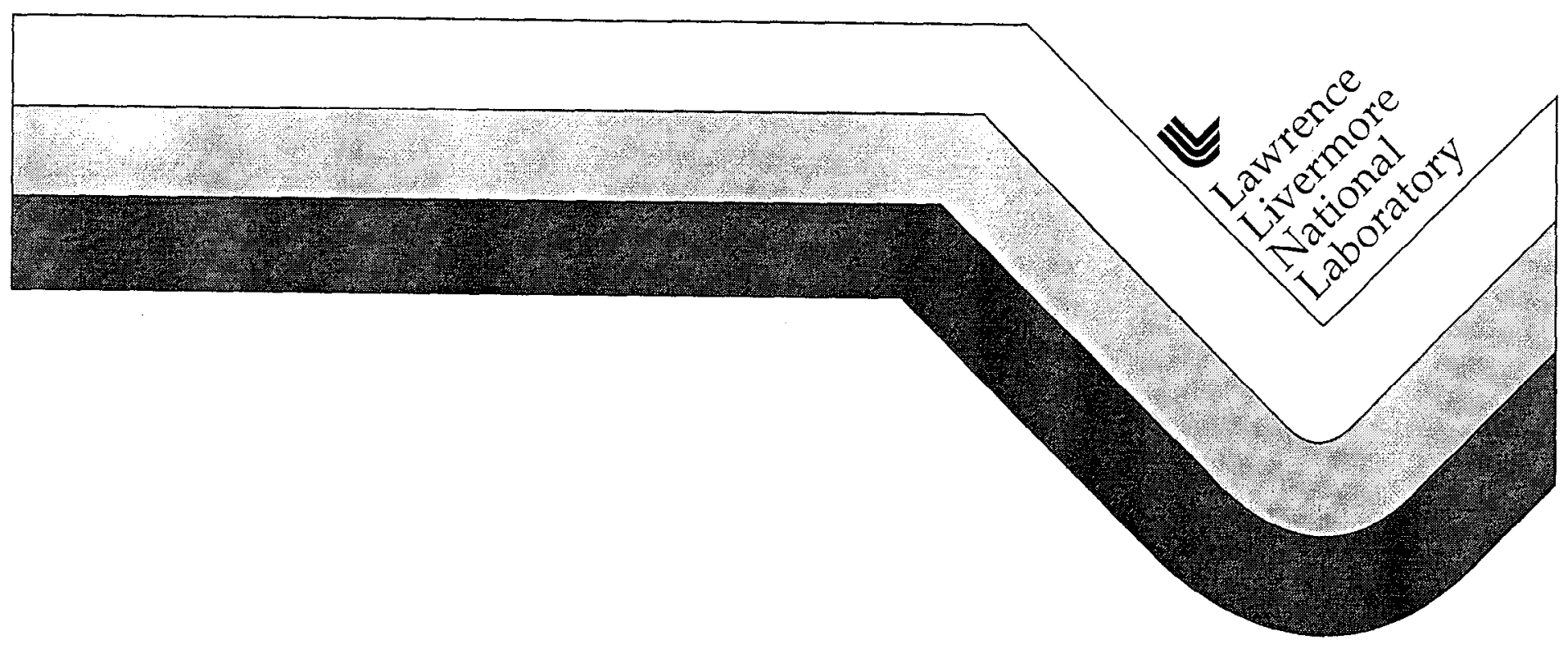




\section{DISCLAIMER}

This document was prepared as an account of work sponsored by an agency of the United States Government. Neither the United States Government nor the University of California nor any of their employees, makes any warranty, express or implied, or assumes any legal liability or responsibility for the accuracy, completeness, or usefulness of any information, apparatus, product, or process

disclosed, or represents that its use would not infringe privately owned rights. Reference herein to any specific commercial product, process, or service by trade name, trademark, manufacturer, or otherwise, does not necessarily constitute or imply its endorsement, recommendation, or favoring by the United States Government or the University of California. The views and opinions of authors expressed herein do not necessarily state or reflect those of the United States Government or the University of California, and shall not be used for advertising or product endorsement purposes. 


\section{A New Mechanical Characterization Method for Thin Film Microactuators and Its Application to NiTiCu Shape Memory Alloy}

Kirk P. Seward

June 1999

LAWRENCE LIVERMORE NATIONAL LABORATORY University of California • Livermore, California • 94551 


\title{
A New Mechanical Characterization Method for Thin Film Microactuators and Its Application to NiTiCu Shape Memory Alloy
}

\author{
by
}

\author{
Kirk P. Seward \\ B. S., Mechanical Engineering \\ Massachusetts Institute of Technology, 1998 \\ Submitted to the Department of Mechanical Engincering \\ in Partial Fulfillment of the Requirements for the Degree of \\ Master of Science in Mechanical Engineering \\ at the \\ Massachusetts Institute of Technology \\ June 1999 \\ (C) 1999 Kirk P. Seward \\ All rights reserved \\ The author hereby grants to MIT permission to reproduce and to distribute \\ publicly paper and electronic copies of this thesis document in whole or in part.
}

Signature of Author.

Department of Mechanical Engineering

May 7, 1999

Certified by

Subra Suresh

Professor of Materials Science and Mechanical Engineering

Thesis Supervisor

Accepted by 


\section{Table of Contents}

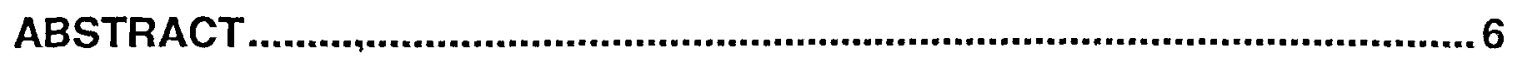

CHAPTER 1: INTRODUCTION AND BACKGROUND ..........................................

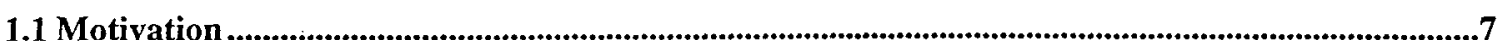

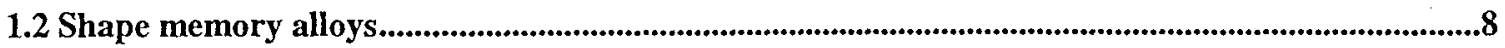

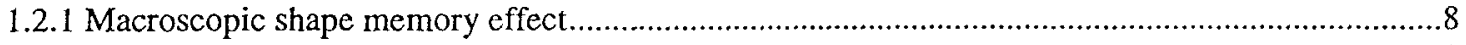

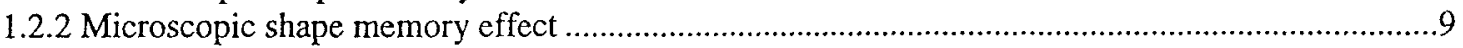

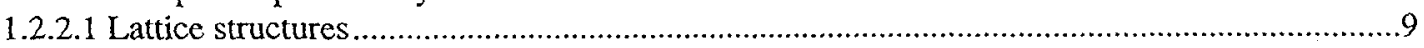

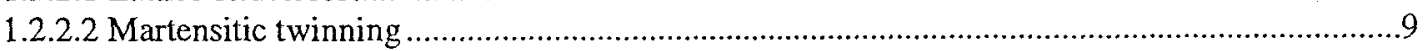

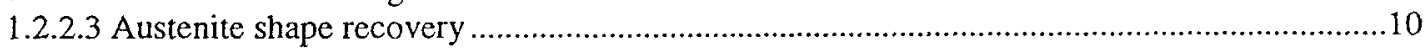

1.3 Microvalves ....................................................................................................................11

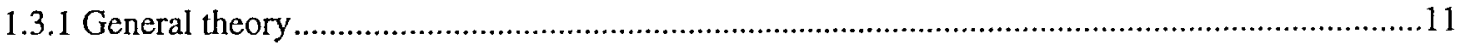

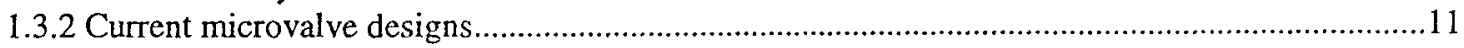

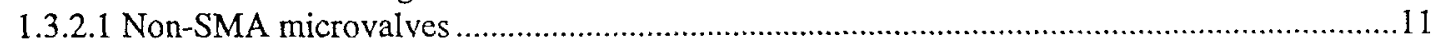

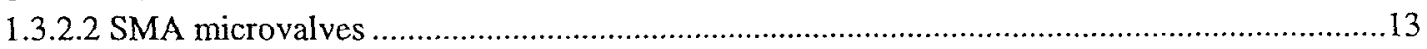

1.4 Applications of microvalves and microactuators...........................................................................14

CHAPTER 2: CHARACTERIZATION TECHNIQUE …....................................17

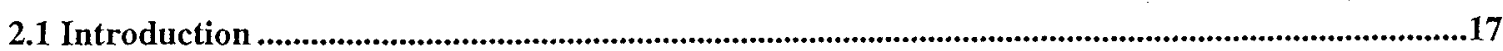

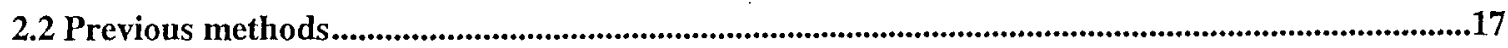

2.3 New approach for SMA characterization..........................................................................................20

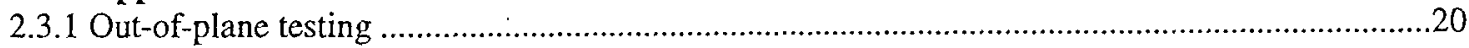

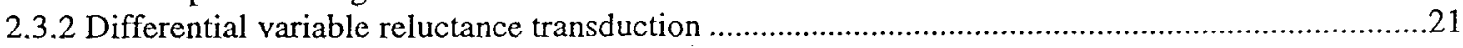

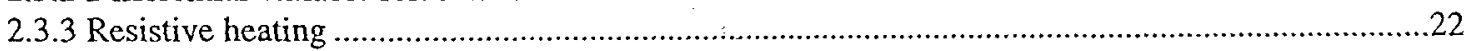

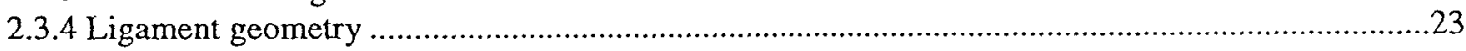

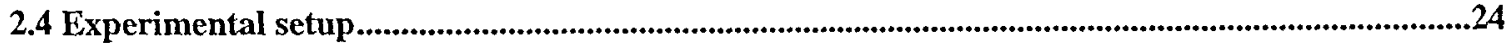

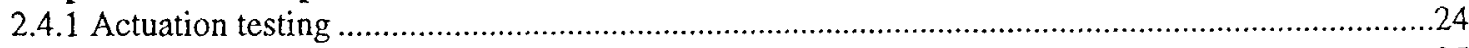

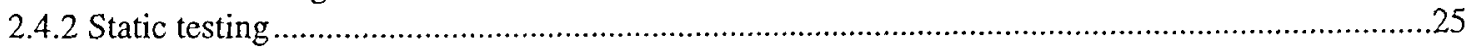

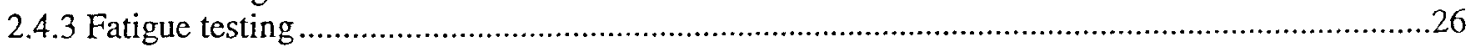

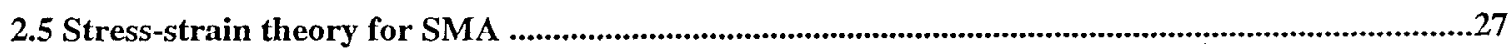

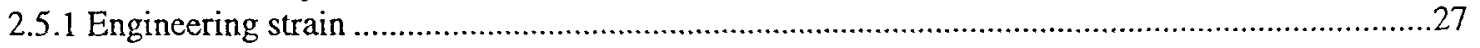

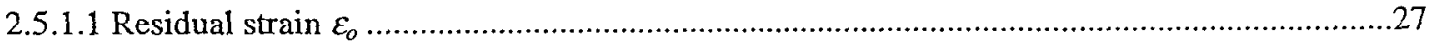

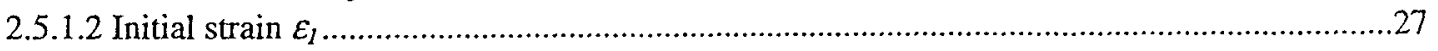

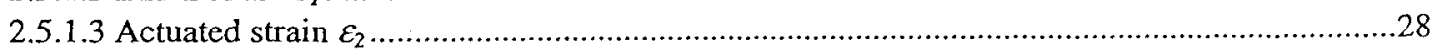

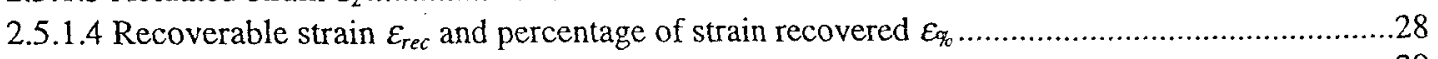

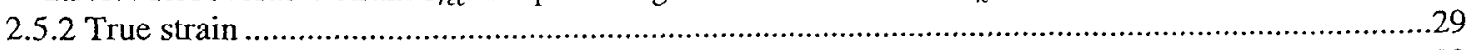

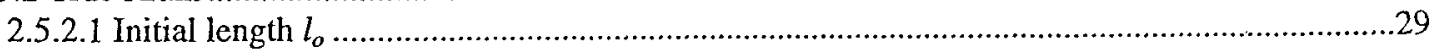

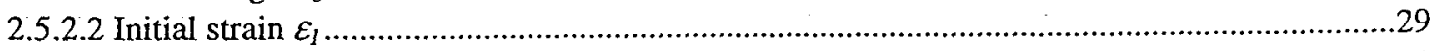

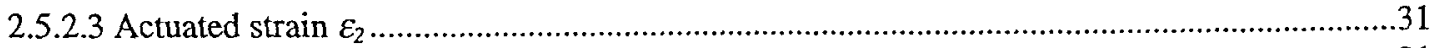

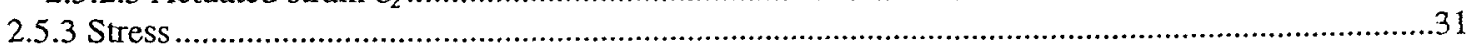

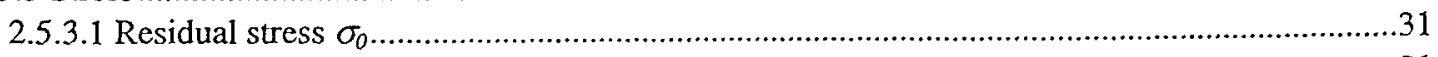

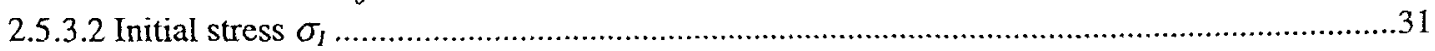

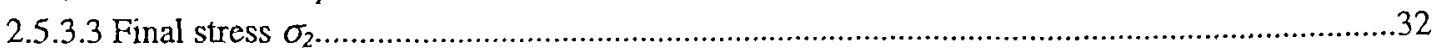

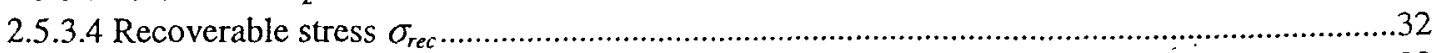

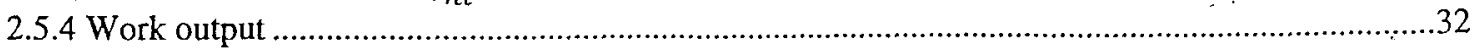




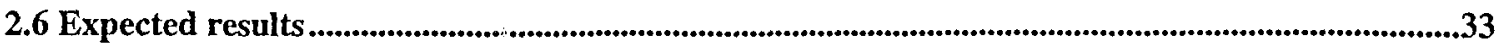

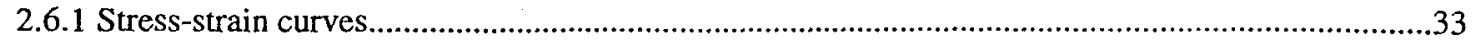

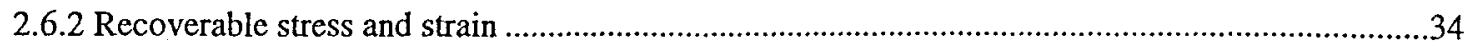

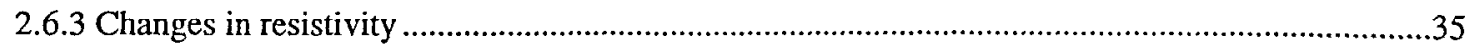

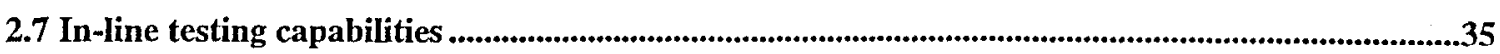

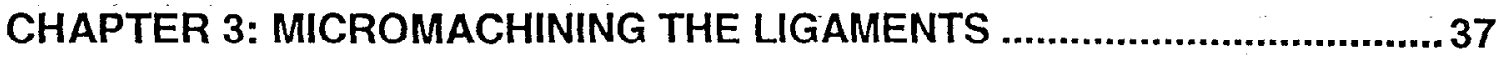

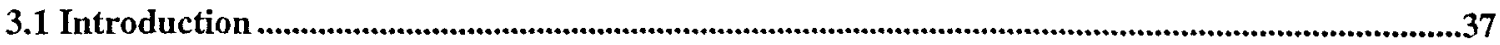

3.2 Successful processing procedure ..........................................................................................................38

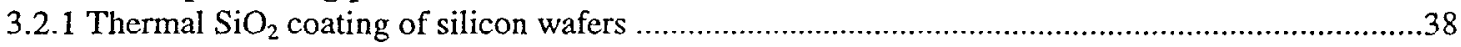

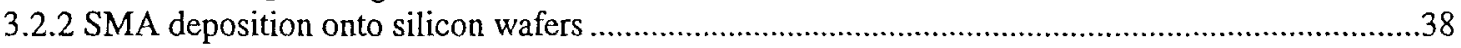

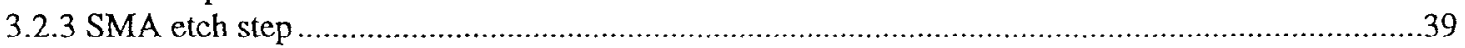

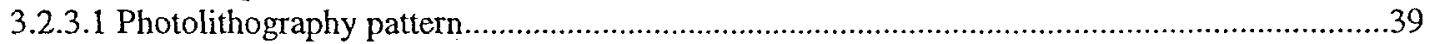

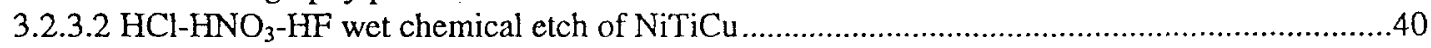

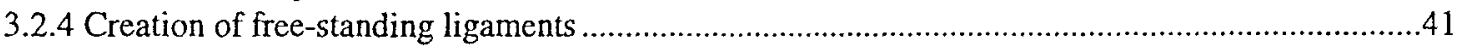

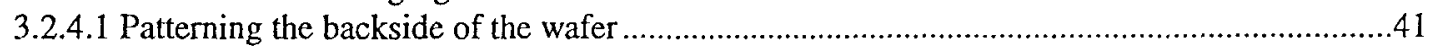

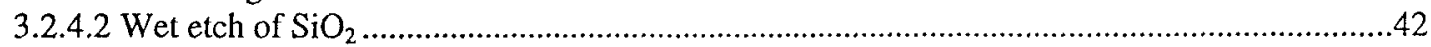

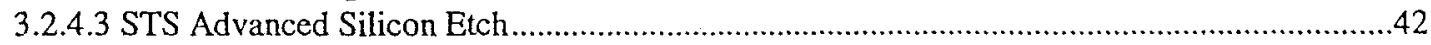

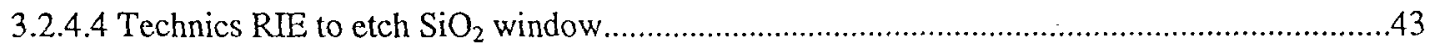

3.3 Unsuccessful wafer processing attempts............................................................................................43

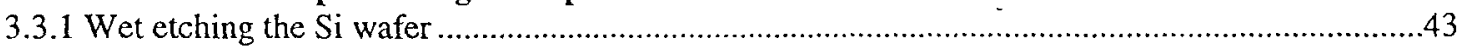

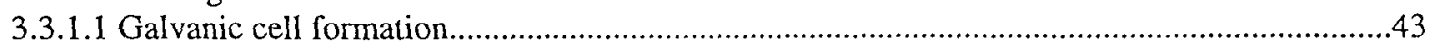

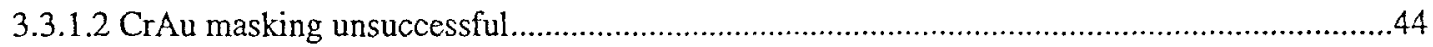

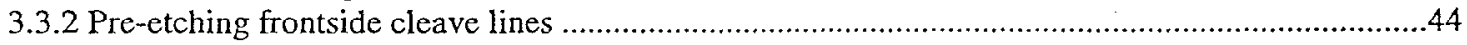

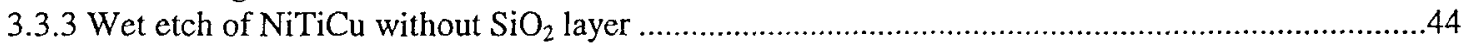

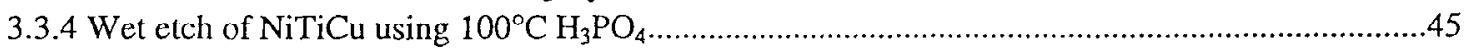

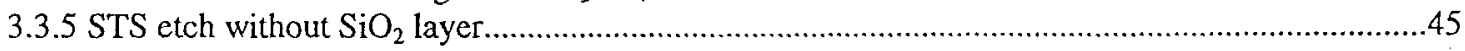

CHAPTER 4: RESULTS AND DISCUSSION .................................................... 46

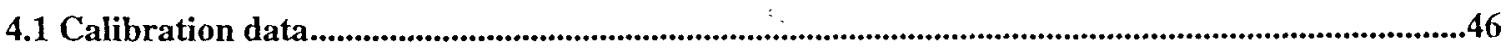

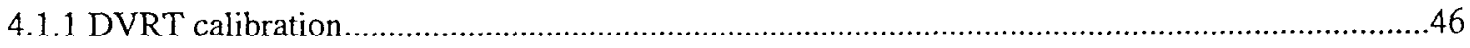

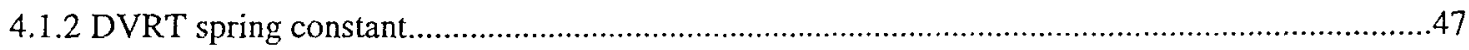

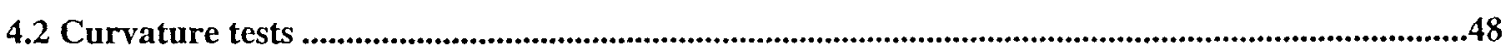

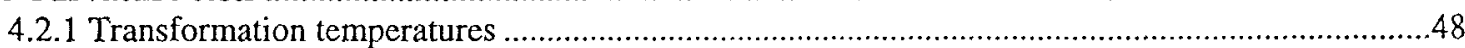

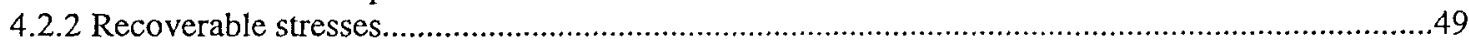

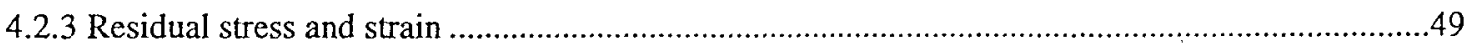

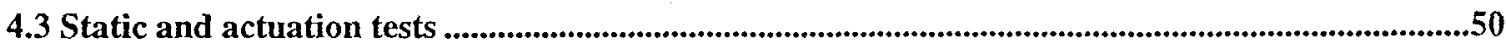

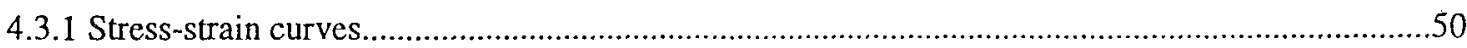

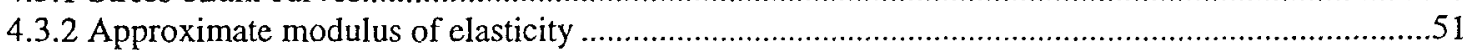

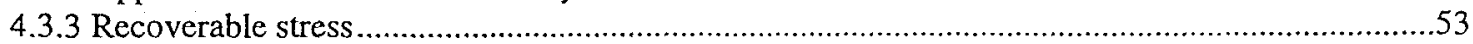

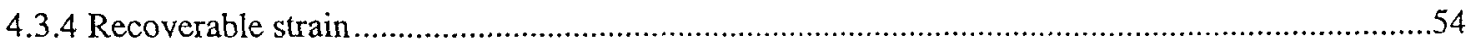

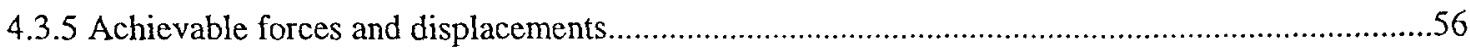

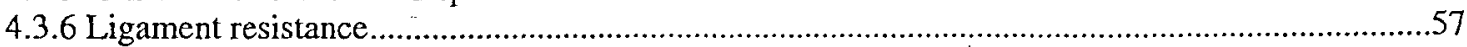

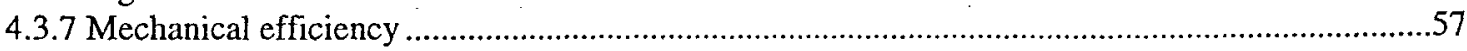

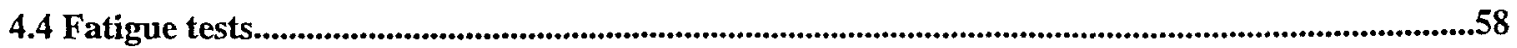

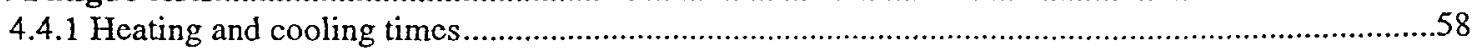

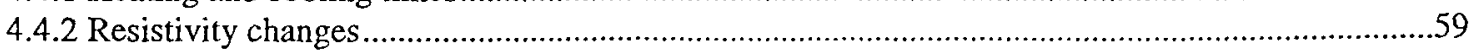

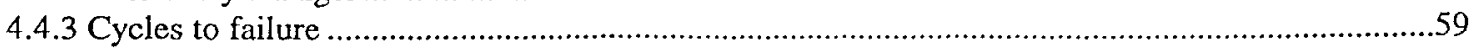

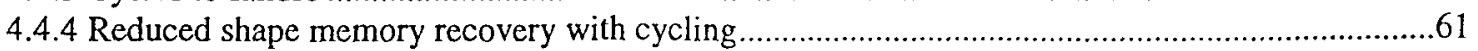

4.4.5 Maximum displacements and forces achievable for long life devices .........................................63 


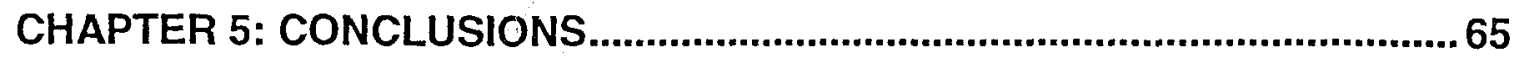

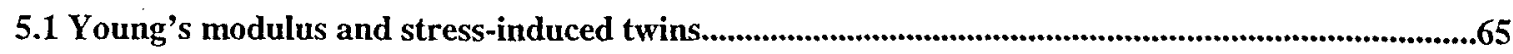

5.2 Cycles to failure .......................................................................................................................................66

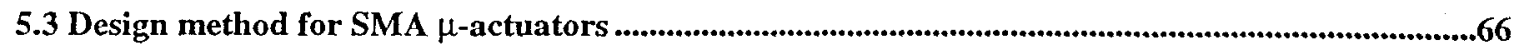

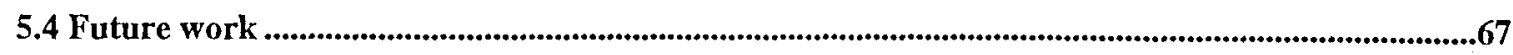

APPENDIX A: UNCERTAINTY ANALYSIS .............................................. 68

APPENDIX B: PARTS LIST AND ASSEMBLY DRAWING FOR TESTING INSTRUMENT .............................................................................................. 71

ACKNOWLEDGEMENTS ....................................................................... 74

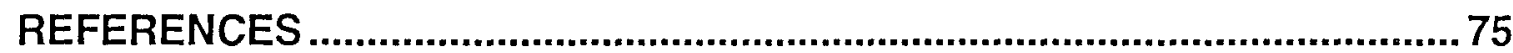




\section{Abstract}

In an effort to develop a more full characterization tool of shape memory alloys, a new technique is presented for the mechanical characterization of microactuators and applied to SMA thin films. A test instrument was designed to utilize a spring-loaded transducer in measuring displacements with resolution of $1.5 \mu \mathrm{m}$ and forces with resolution of $0.2 \mathrm{mN}$. Employing an out-of-plane loading method for freestanding SMA thin films, strain resolution of $30 \mu \varepsilon$ and stress resolution of $2.5 \mathrm{MPa}$ were achieved. This new testing method is presented against previous SMA characterization methods for purposes of comparison.

Four mm long, $2 \mu \mathrm{m}$ thick NiTiCu ligaments suspended across open windows were bulk micromachined for use in the out-of-plane stress and strain measurements. The fabrication process used to micromachine the ligaments is presented step-by-step, alongside methods of fabrication that failed to produce testable ligaments. Static analysis showed that $63 \%$ of the applied strain was recovered while ligaments were subjected to tensile stresses of $870 \mathrm{MPa}$. In terms of recoverable stress and recoverable strain, the ligaments achieved maximum recovery of $700 \mathrm{MPa}$ and $3.0 \%$ strain. No permanent deformations were seen in any ligament during deflection measurements. Maximum actuation forces and displacements produced by the $4 \mathrm{~mm}$ ligaments situated on $1 \mathrm{~cm}$ square test chips were $56 \mathrm{mN}$ and $300 \mu \mathrm{m}$, respectively. Fatigue analysis of the ligaments showed degradation in recoverable strain from $0.33 \%$ to $0.24 \%$ with 200,000 cycles, corresponding to deflections of $90 \mu \mathrm{m}$ and forces of $25 \mathrm{mN}$. Cycling also produced a wavering shape memory effect late in ligament life, leading to broad inconsistencies of as much as $35 \%$ deviation from average.

Unexpected phenomena like stress-induced martensitic twinning that leads to less recoverable stress and the shape memory behavior of long life devices are addressed. Finally, a model for design of microactuators using shape memory alloys is presented to illustrate how results obtained from these tests can be interpreted and applied to the creation of MEMS devices. 


\section{Chapter 1: Introduction and background}

\subsection{Motivation}

Following the trend of decreased size for increased functionality, mechanical systems have taken advantage of Integrated Circuit (IC) technology's surface machining and bulk micromachining to produce the field of Micro Electromechanical Systems (MEMS). Micromachine components in the form of pumps, valves, and actuators create intricate systems that allow control and manipulation of fluids through fluidic circuitry, and micromachines through tiny gears and linkages.

In an effort to create more viable and efficient microactuators, many systems of motion have been investigated, including piezoelectrics, electrostatics, magnetics, and shape memory alloys (SMA's). The shape memory effect of Nickel-Titanium (NiTi) alloys was discovered and patented by the United States Navy in the 1940's under the name Nitinol [source]. This material was used for many years and has many applications in its bulk form [sources], but has also taken the form of thin films in the industry of microtechnology for use in actuation devices. While patents exist for SMA microvalves and microactuators [source], the mechanical characterization of material properties of these SMA films is not complete. Researchers have worked to understand recoverable stresses and strains, temperatures at which phase transformations occur, and crystallographic microstructure and texture. In parallel, mathematicians have worked to create a numeric model that will predict the physical behavior of SMA films and incorporated the findings of researchers to test the success of their models. Until now, however, no scientist or engineer had developed a single test that can determine mechanical properties like recoverable stresses and strains and material properties like resistivity at the same time while under a load, a test that reveals the material properties necessary for designing actuators.

In an effort to further understand the behavior of SMA films under loads, a testing apparatus was designed to hold films under tension while actuating them to determine their mechanical reactions when employed in real microdevices. This test setup has utility in the design of SMA film microactuators to predict their response by measuring forces and displacements possible from actuators and to monitor the quality of the deposited film from batch to batch during fabrication. In this paper, the applications of the new testing instrument to mechanically characterizing SMA films sputtered from nickel-titanium-copper (NiTiCu) targets is examined in full detail. 


\subsection{Shape memory alloys}

\subsubsection{Macroscopic shape memory effect}

Shape memory alloys are appropriately named because they "remember" their shape when a solid to solid phase transformation occurs during heating. At room temperature, $\mathrm{NiTiCu}$ is in its low-temperature, martensite state. When it is heated beyond its transformation temperature, it undergoes the phase transformation to an austenite state. In the martensitic state, the material exhibits higher ductility than the austenitic state and is therefore easily deformed. When the material is heated and enters its austenite phase, it regains its original shape as long as plastic deformation did not occur during martensite (around 4-10\% strain for most SMA's [source]). Upon cooling to martensite again, the NiTiCu does not change shape again unless external loads are applied. Other SMA's can experience a two-way shape memory effect in which both phases remember a unique shape [source], but that issue was not confronted in this research. Observations of decreased electrical resistance in the austenite phase made by other researchers [source] were also confirmed by this study.

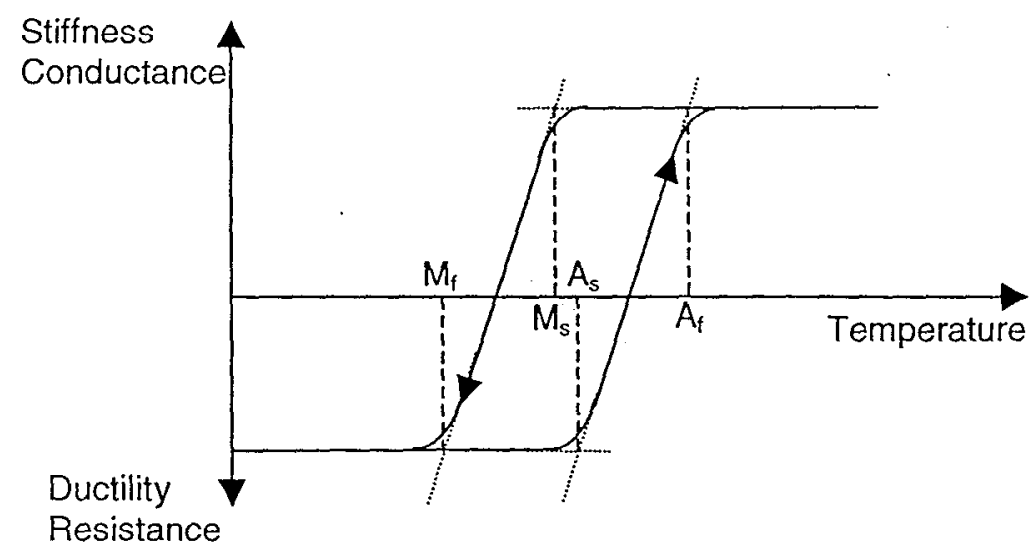

Figure 1.I: Shape memory effect hysteresis

Phase transformations in the shape memory effect are hysteretic and are therefore characterized by start and finish temperatures for each phase, as illustrated by Figure 1.1. In this figure, the four pertinent transformation temperatures are upon heating: the austenite start temperature, $A_{s}$ and the austenite finish temperature, $A_{f}$; and upon cooling: the martensite start temperature, $\mathrm{M}_{\mathrm{s}}$ and the martensite finish temperature, $\mathrm{M}_{\mathrm{f}}$. Hysteresis width and transformation temperatures have shown empirical dependence on alloy composition and crystal microstructure, so they are normally consistent as long as fabrication methods are not variable. Results from different researchers are hard to correlate, however, because of different methods of film deposition and what kind of micromachining subsequently takes place. 


\subsubsection{Microscopic shape memory effect}

\subsubsection{Lattice structures}

Shape memory transformations occur by solid-to-solid phase transitions from one crystal lattice structure to another, sometimes with an intermediate phase during heating or cooling. The austenite phase, or parent phase, has a tightly packed body centered cubic (BCC) lattice while the martensite phase is monoclinic or orthorhombic subject to alloy composition. NiTi martensite is monoclinic and NiTiCu martensite is orthorhombic. The three lattice structures are depicted by Figure 1.2 for both NiTi and NiTiCu. During the NiTi phase transformation upon cooling from austenite to martensite, the alloy passes through its intermediate R-phase, which has been described as both rhombohedral and orthorhombic by different scientists. The rhombohedral lattice structure is thercfore also depicted in Figure 1.2.

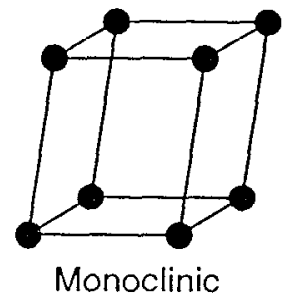

Monoclinic

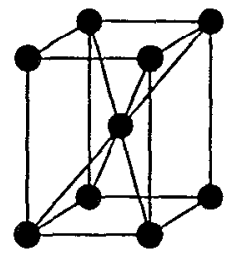

Body Centered Orthorhombic

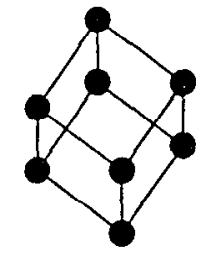

Rhombohedral (Trigonal)

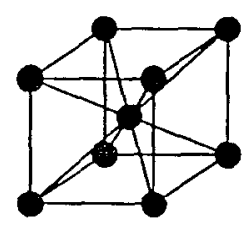

Body Centered Cubic

Figure 1.2: Pertinent crystal lattice structures used in the study of Ni-Ti-based SMAs.

\subsubsection{Martensitic twinning}

The martensite phase has been described by the author as ductile, but not plastic in its deformation. The reason behind this is the twinned structure encountered in martensite. Twins are formed by crystals in the material, then twins of those crystal twins form, and twins of those twins and so forth. This leads to a very complex microstructure, with twinning dimensions much less than the material dimensions. For MEMS applications, the twinning dimensions are on the order of nanometers, while the material dimensions are on the order of microns. This highly twinned structure is ductile because twin planes are able to slip with respect to one another. Since twins are moving with respect to each other, dislocations do not appear and there is no plastic deformation. This leads to the method of interpreting the the presence of twins in NiTi based shape memory alloys, as reported by Bhattacharya [reference]:

1. The lattice on one side can be obtained by a simple shear of the lattice on the other.

2. The lattice on one side can also be obtained by a rotation of the lattice on the other. 
These conditions are at the heart of twin variants. When lattices are sheared or rotated, they transform from one variant of the martensitic lattice structure into another. Thus, variants change because of the twinned microstructure, which appears as macroscopic plastic deformation. Bhattacharya goes on to report the reason for the formation of this martensitic microstructure. The crystal lattice structure "prefers" to exist at low energy, or at the bottom of its energy well. The kinematic compatibility of the material states that the deformation of the material must be coherent, so macroscale martensitic material appears to have the same properties throughout, though it is made up of several variants of one lattice structure [reference].

Martensitic material also takes on the material property known as self-accommodation. This property allows for no macroscopic change in shape in the absence of external loads when the material transforms from austenite to martensite. It states that during phase transformation, the new martensite variants align themselves with one another to allow no macroscopic shape change in the material. This is also explained by the energy balance of the transformation. During the austenite to martensite transformation, the material emits heat and the transformation is exothermic. The material would obviously require energy to undergo a change in shape, and therefore the variants undergo extremely small changes at the scale of lattice and microstructure to accommodate the extremely small strains present in the microstructure around them.

\subsubsection{Austenite shape recovery}

All shape memory materials start out as austenite. Before annealing, the materials have amorphous microstructures without order or aligniment of crystal lattices. Upon annealing, the highly symmetric BCC crystallization takes place, forming SMA austenite. Then when it cools, it enters martensite for the first time and undergoes self-accommodation. When the NiTiCu enters the austenite phase, twins are recovered during the transition to BCC. As the multiple twin variants transform to the unique $\mathrm{BCC}$ variant, the material recovers its original shape. In essence, the material loses its twins during this transformation as all existing lattice structures that were created by strain and self-accomodation coalesce into one structure, dictated by the austenite lattice parameters. If strain was applied to the martensitic structure, causing deformation, the transformation back to BCC causes a macroscopic shape change to its original, annealed shape. This is an endothermic process, as energy is absorbed from the heat source to cause the lattice transition and nanoscale motion of atoms necessary for the phase transformation. 


\subsection{Microvalves}

\subsubsection{General theory}

Microvalves have been fabricated using Si wafer $\mu$-machining techniques since the mid1980s. They are used to manipulate small amounts of fluid on the order of $\mathrm{mL} / \mathrm{min}$. The benefits of these microvalves are low power requirements and small dead volumes, allowing more accurate fluid control. By stacking wafers and $\mu$-machining channels, micropumps and microvalves, fluidic circuits in which different fluids are input, guided and mixed together, and output are made possible.

As a general rule, microvalves have many similar components, regardless of how they are made or what actuation method they involve. Components are described as follows and seen in Figure 1.3. The boss of the valve is the mass that can be moved to obstruct or allow flow through the valve by being pressed against or moved away from the valve seat. The valve seat is usually machined from a silicon wafer or similar substrate. Fluid flows into the valve through the inlet and out through the outlet. The valve actuator is the mechanism that moves the boss by deflecting the $\operatorname{leg}(s)$ of the valve. Normally open valves allow flow when not actuated and obstruct flow when powered. The antithesis is true for normally closed valves, staying closed when not powered. Unless the valve is two-way, the valve seat surrounds the valve outlet, so that the boss is not pushed off of the seat by the input pressure, which would allow leaking.

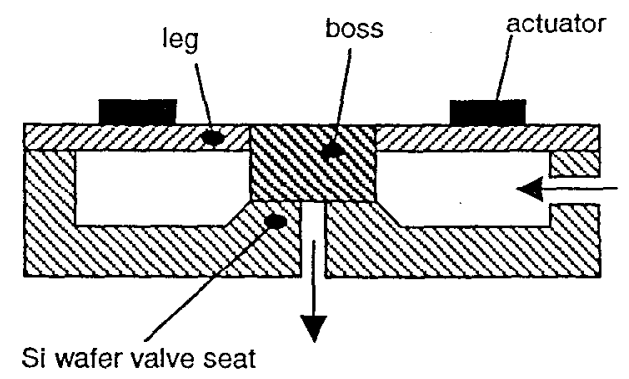

a) Normally closed: actuation pulis boss up from valve seat

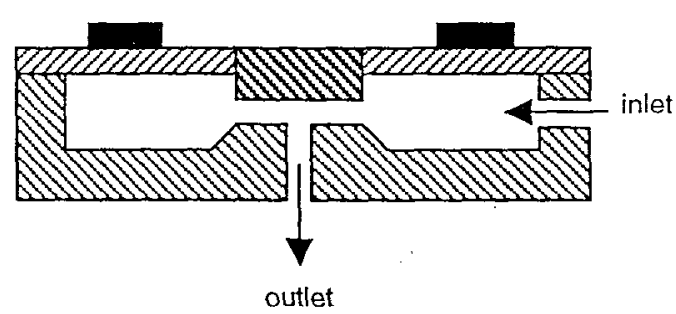

b) Nomally open: actuation pushes boss down onto valve seat.

Figure 1.3: Typical structure and components of normally open and normally closed microvalves.

\subsubsection{Current microvalve designs}

\subsubsection{Non-SMA microvalves}

Four microvalves are presented here to serve as a reference point for the SMA microvalves examined later in this chapter. All of the valves are actuated by electrical means, but two involve some form of resistive heating, while the other two operate directly from the current 
applied to their actuative mechanisms. Schematic diagrams of the four valves are seen in Figure 1.4 , both in open and closed orientations.

The first valve, seen in Figure 1.4(A), is Redwood Microsystems Fluistor ${ }^{\mathrm{TM}}$ Sealed Capsule Expansion Valve [reference]. This valve consists of a single piece of machined silicon bonded to two glass wafers, one of which has been machined with through holes for fluidic connections. The fluid that is trapped between the silicon and unmachined glass wafer evaporates and expands when heated by a patterned resistor in contact with the fluid, causing the silicon diaphragm to bend and dislocate the boss from the valve seat. This is a normally closed valve that is capable of controlling $2000 \mathrm{sccm}$ of air or nitrogen at $25^{\circ} \mathrm{C}$ and $100 \mathrm{psid}$ of pressure. The response time of this valve is rather slow, however, at $400 \mathrm{~ms}$.

Next is the EG\&G IC Sensors Al/Si bimetal valve [reference], which operates by the coefficient of thermal expansion mismatch between the patterned aluminum film and the thin silicon cantilevers. When the legs of this structure are heated resistively, the aluminum expands more than the silicon, forcing the legs to buckle under the load and raise the boss from the valve seat. This microvalve is better for small flows, operating well at $100 \mathrm{sccm}$ of air or nitrogen at $25^{\circ} \mathrm{C}$ and 25 psid of pressure. The response time of this valve $(100 \mathrm{~ms})$ is also shorter than the Redwood valve because less time is necessary to heat the metal film than the encapsulated fluid.

The third valve investigated is an electrostatically driven gas valve developed by Hitachi [reference]. This valve operates by switching the flow from one outlet port to the other whenever necessary. Electrostatic actuation occurs by passing a current through the gap between the flexible iron-nickel film and the silicon conductors, causing a capacitance and an attractive force between the film and the active conductor. Switching the current from one conductor to the other allows the changing of flow from one orifice to the other. While response times for this valve were not documented, it can handle gas flows at a rate of $1 \mathrm{sccm}$ under pressures less than $100 \mathrm{~Pa}$.

The final non-SMA wafer presented here is the magnetically actuated flow valve developed by R. L. Smith et. al. at UC Davis [reference]. This valve is actuated by creating a magnetic field normal to the plane of the polysilicon stopper, which attracts the magnetic material and closes the valve. The magnetic field is created by an electromagnet mounted above the valve outlet, which could theoretically switch the stopper to cover either opening of the valve. A normally open valve, this is expected to contain $\mathrm{Cr}$ steel for the magnetic material, with a residual magnetic field of 10,000 Gauss such that switching between states would employ a total current of $200 \mathrm{~mA}$. No flow predictions were included in the literature, but sealing pressures of $50 \mathrm{kPa}$ and switching times of a few milliseconds were expected from theoretical calculations. 

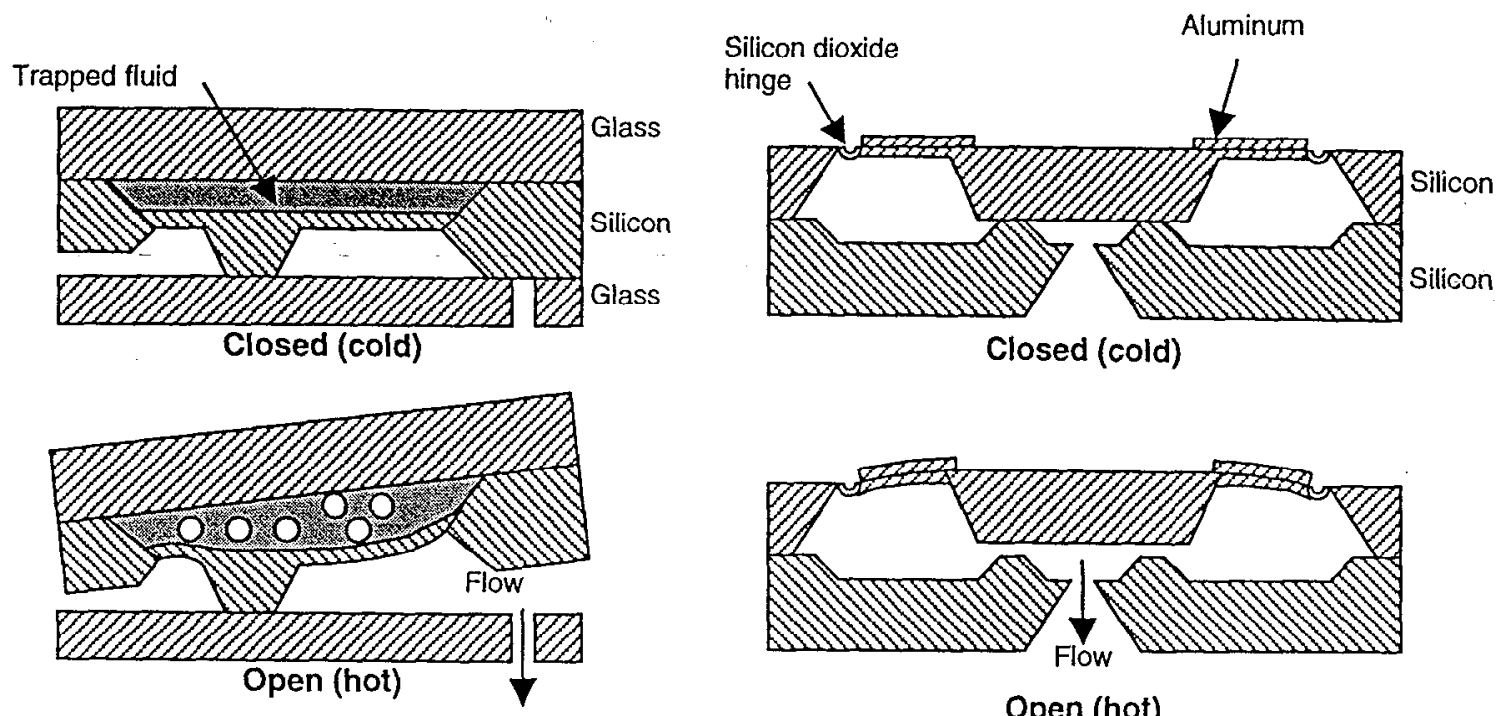

Closed (cold)

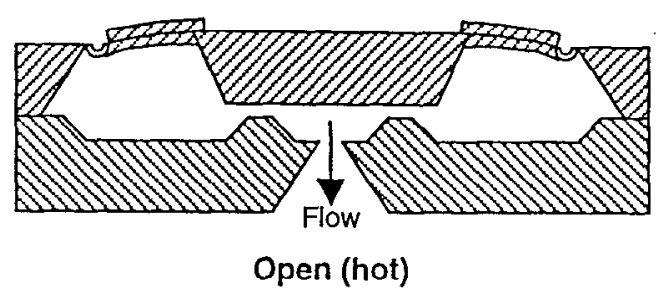

A: Redwood Microsystems Fluistor ${ }^{\mathrm{TM}}$ Sealed Capsule Expansion Valve
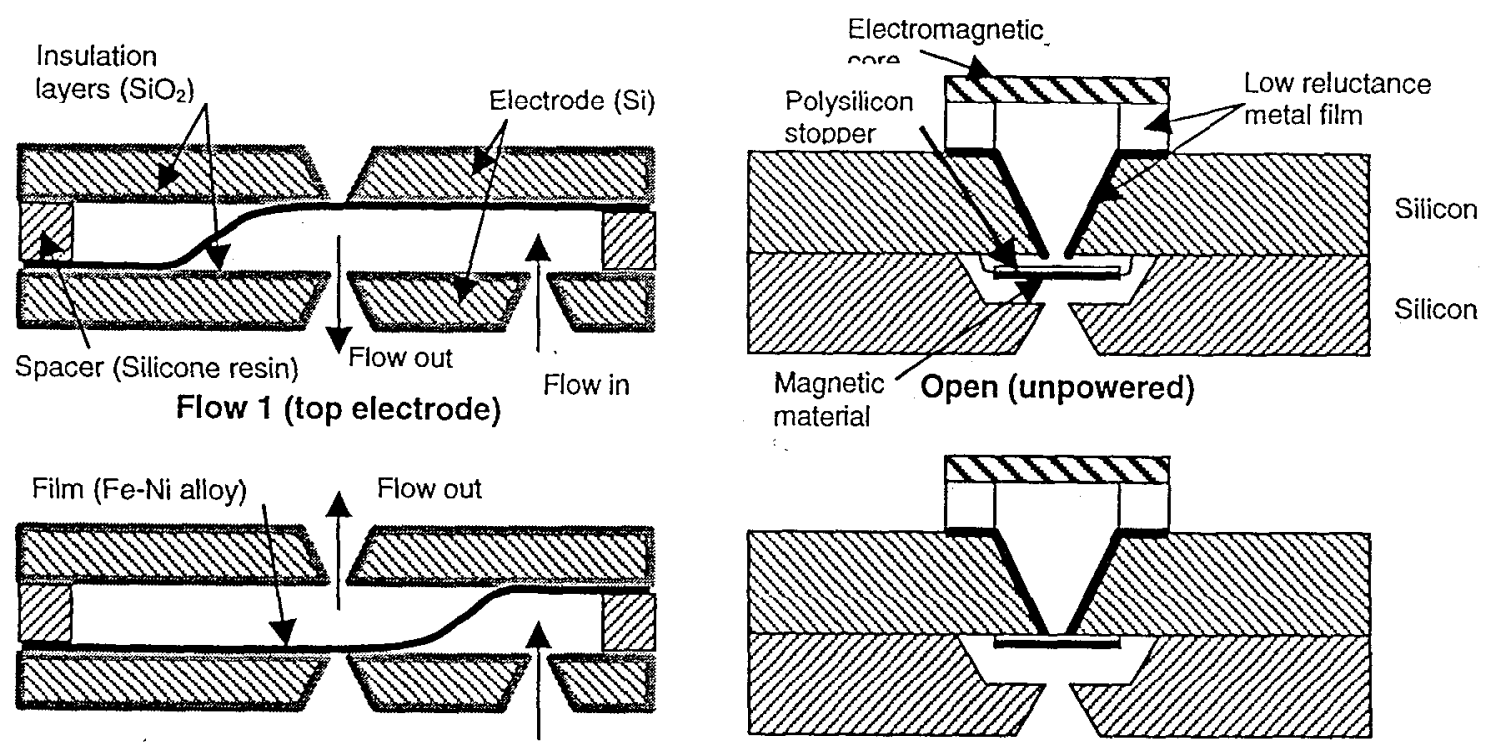

Flow 2 (bottom electrode) Flow in

C: Hitachi Electrostatically Driven Gas Valve

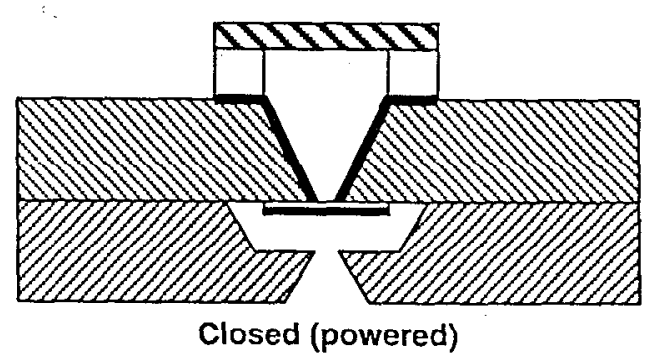

D: Magnetically Actuated Flow Valve by R. L. Smith, et. al., UC Davis

Figure 1.4: Examples of non-SMA microvalves. $A$ and $B$ actuate by heating and $C$ and $D$ actuate electrically.

\subsubsection{SMA microvalves}

To this researcher's knowledge, the only microvalve that employs thin film shape memory alloy for actuation was designed and patented by the TiNi Alloy Company [reference]. The method they have used to create actuation, illustrated in Figure 1.5, was to use a berylliumcopper bias spring to deflect a boss downward by strctching the thin TiNi ribbons that connect it 
to the main structure of the valve. As current is run through these TiNi ribbons, they heat resistively and undergo their phase transformation from the ductile martensite to the stiff austenite and try to regain their original shape, or length in this case. When current is removed from the alloy, it cools and the spring deflects it again, closing the valvc.
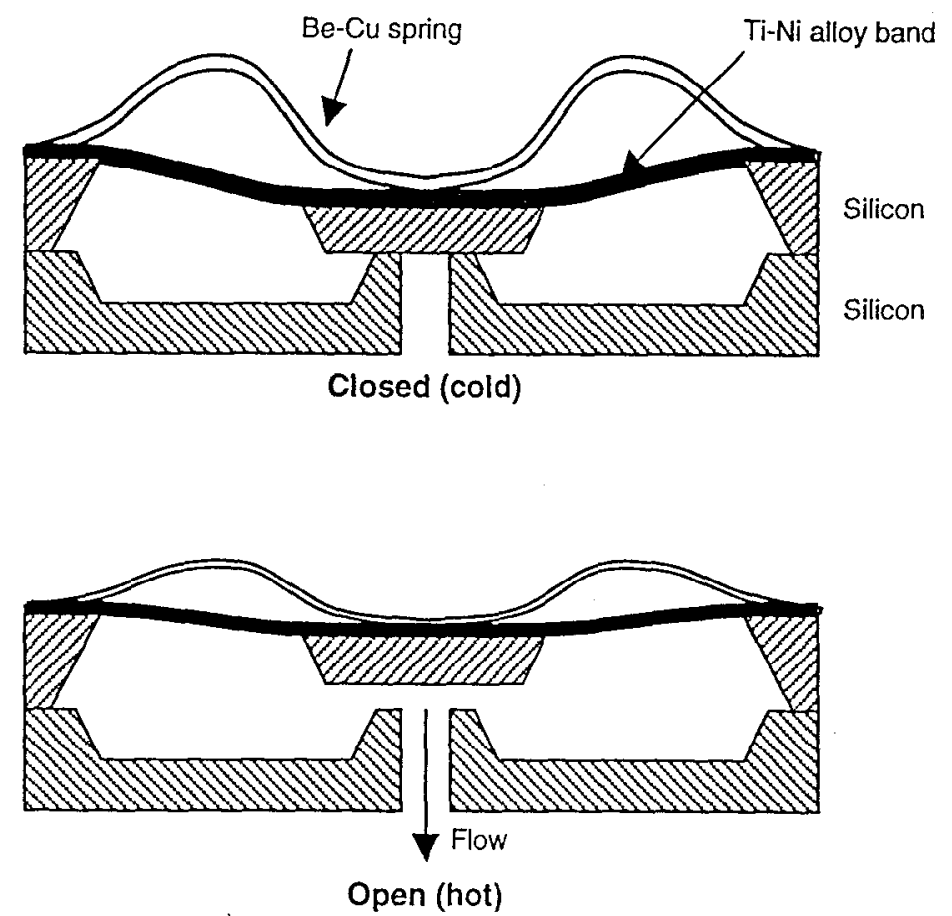

Figure 1.5: The TiNi Alloy Company developed and patented a microvalve that employs freestanding shape memory alloy ribbons acting against a beryllium-copper spring to cause actuation.

The SMA test structures described in this paper were modeled after these microvalves by creating thin film ligaments from $\mathrm{NiTiCu}$ and deflecting them with a spring-transducer that could measure their reaction force during martensite and austenite phases. The material characterization produced by this research is a valuable tool for analyzing structures like the TiNi microvalve and creating new structures that actuate in a similar fashion with freestanding SMA ligaments or ribbons. This paper and some literature refer to ligaments where other literature refers to ribbons, but they are the same structures, differing in name only.

\subsection{Applications of microvalves and microactuators}

One very interesting application of microvalves and microactuators is the creation of micropumps. Two check valves are placed in series with a diaphragmatic actuator, one on each side, creating a pumping system. When the diaphragm is actuated in one direction, it pulls fluid into a cavity through one check valve, while the other is held shut. As the diaphragm actuates in the other direction, the fluid is pushed out of the pump through the second check valve while the 
first is held shut. One way that micropumps could be created using shape memory thin films is by film deposition on an entire diaphragm and actuation by heating the film, bending the diaphragm, and pulling and pushing fluid into and out of the pump.

When microvalves and micropumps are combined into integrated devices, fluidic circuits are created. Shape memory alloy deposition can be utilized to fabricate several devices on one silicon wafer, with channels running between the separate modules, to create systems for mixing chemicals, sorting DNA, and numerous other applications.

Microvalves can also be utilized in applications such as gas chromatographs, mass flow controllers, ink-jet print heads, refrigeration systems, automobile fuel systems, and droplet ejectors for biomedical applications. To date, substandard valves have been created, but no spectacular microvalve has been developed for use in these applications

Abraham Lee, et. al., of Lawrence Livermore National Laboratory, developed a very useful microdevice for use in biomedical applications. Their microgripper relies on a thin film of $\mathrm{NiTiCu}$ that was sputtered onto a solid silicon substrate, which causes the substrate to bend as the film is heated, due to the residual stress of the austenite film [reference]. The creation of a double-cantilever geometry, with one cantilever as the mirror image of the other, as seen in Figure 1.6, and the bonding of these sections together created a structure that, when heated, opens up like a jaw. This device can be used to grip arterioveinal stints for placement or removal or to chew away at blockages in arteries or veins, thus allowing less constricted blood flow.

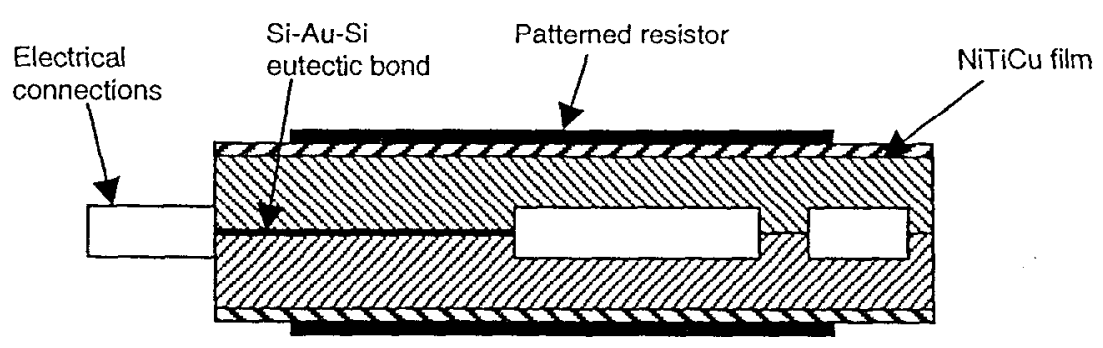

Figure 1.6: The microgripper developed by A. P. Lee of Lawrence Livermore National Laboratory utilizes the residual stress difference between the martensitic and austenitic phases of NiTiCu to open its jaws by heating with patterned resistors.

Yet another application of freestanding SMA ligaments to MEMS devices was developed by Kris Pister at the University of California at Berkeley [reference]. While working with hinged microstructures created by surface micromachining, the problem was faced with how to pull a nominally horizontal structure to a vertical position and keep it there. Through sputter deposition and patterning of SMA, freestanding alloy bridges could be created that attach to the hinged microstructure, as seen in the first part of Figure 1.7. While in martensite, these freestanding 
bridges undergo some strain because of the mismatch of coefficients of thermal expansion. Since more residual austenite stress exists in the structures at this point, heating causes the bridges to contract and pull up the horizontal structure into its vertical orientation. Hard stops are implemented at this point for positioning at whatever angle is desired.
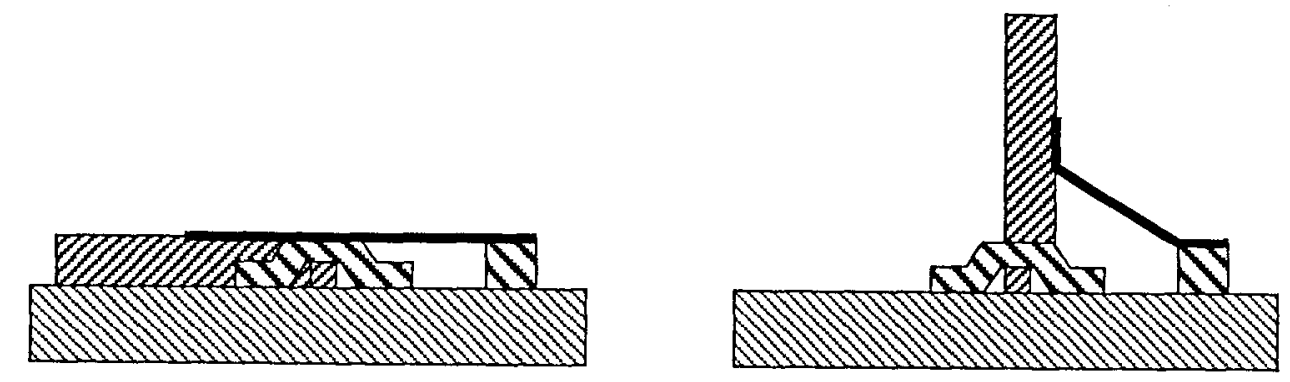

Figure 1.7: K. Pister of UC Berkeley has enabled reorientation of hinged structures by heating freestanding SMA ligaments that contain residual austenite stresses. 


\section{Chapter 2: Characterization technique}

\subsection{Introduction}

While concepts exist to use SMAs in microvalves, pumps and actuators, alloys have not yet been characterized well enough to effectively design MEMS that utilize their actuation properties. Because SMA films behave differently than bulk materials and vary in mechanical properties with distinct fabrication methods, a simple technique to provide all necessary mechanical data is beneficial.

Alloys have not been well characterized because the material's behavior varies with small changes in deposition methods and alloy composition. Many researchers have concentrated their efforts on creating mechanical and mathematical models to predict the behavior of these alloys [references], but due to minute differences in material behavior, difficulty arises in the correlation of data. The test described here is non-destructive and provides a comprehensive range of thermomechanical information with simultaneous determination of stress-strain behavior and material resistivity of both phases, recoverable stress against a load, fatigue behavior, response and cycling times, actuator displacement, and power requirements for actuation of $\mathrm{NiTiCu}$ ribbons. Performing a comprehensive engineering analysis of this material facilitates the design of MEMS devices that employ SMA films.

\subsection{Previous methods}

The actuation possible from SMAs relies upon the solid-solid phase transformation described in Section 1.2. Because of this material transformation, the alloy's twin structure, stiffness (Young's modulus), resistivity, and energy balance change with heating and cooling beyond transformation temperatures. Each of these properties helps to fully characterize the alloy, but no individual property can explain everything needed for design. Previous experimental methods and their measurement capabilities are compared in Table 1. These techniques individually help to find transformation temperatures, electrical properties, mechanical data, thermal characteristics, and microstructure. There is no prior method, however, that encompasses as many properties as the new characterization method presented in this paper.

The mini-tensile test on NiTiCu ribbons was developed by Suichi Miyazaki and others [references]. In their research, NiTi or NiTiCu films were deposited onto glass substrates, patterned into tensile members (\#\# $\mathrm{mm}$ x \#\# $\mathrm{mm}$ ) for stress testing and square members (\#\# $\mathrm{mm} \mathrm{x}$ \# $\mathrm{mm}$ ) for calorimetry. The films were then freed from the glass for use in testing. Tensile members were pulled to specified strains, unloaded, and heated to determine how much of the 
strain could be recovered. Results from these tests showed that perfect shape memory, with full strain recovery, occurred at up to \#\# strain in \#\#m thick ribbons.

Table 1: Comparison of existing SMA characterization methods with new approach.

\begin{tabular}{|c|c|c|c|c|c|c|}
\hline Testing Method & $\begin{array}{c}\text { Transformation } \\
\text { Temperatures }\end{array}$ & $\begin{array}{c}\text { Electrical } \\
\text { Resistivity }\end{array}$ & $\begin{array}{c}\text { Micro- } \\
\text { Structure }\end{array}$ & $\begin{array}{c}\text { Stress-Strain } \\
\text { Characteristics }\end{array}$ & $\begin{array}{c}\text { In-line } \\
\text { Testing }\end{array}$ & $\begin{array}{c}\text { Non- } \\
\text { destructive }\end{array}$ \\
\hline $\begin{array}{c}\text { Mini-tensile } \\
\text { test on NiTicu } \\
\text { ribbons }\end{array}$ & No & Possible & No & Yes & No & No \\
\hline $\begin{array}{c}\text { Scanning } \\
\text { calorimetry }\end{array}$ & Yes & No & Limited & No & No & No \\
\hline Film resistivity & Yes & Yes & Limited & No & Possible & Yes \\
\hline $\begin{array}{c}\text { Wafer } \\
\text { curvature }\end{array}$ & Yes & No & No & Limited & $\begin{array}{c}\text { Not on } \\
\text { actual parts }\end{array}$ & Yes \\
\hline $\begin{array}{c}\text { Diaphragm } \\
\text { bulge tests }\end{array}$ & No & No & No & Yes & Possible & Yes \\
\hline T.E.M. & No & No & Yes & No & No & No \\
\hline New approach & No & Yes & Limited & Yes & Yes & Yes \\
\hline
\end{tabular}

Miyazaki's group and other researchers [references] have used differential scanning calorimetry (DSC) to find transformation temperatures of SMA films. Calorimetry is useful in finding transformation temperatures because the energy balance of SMA during transformation is shifted away from normal. During the transition from austenite to martensite the material is endothermic, absorbing heat energy to cause the shift from twinned to BCC material. As the SMA cools and enters the martensite phase, it is exothermic during the shift, losing energy and allowing the material to become less stiff. The heat exchanges of the material are seen during DSC, showing the transformation temperatures and hysteresis width of the SMA.

Film resistivity has been measured while heating and cooling SMAs to monitor changes in resistance from one phase to the other [references]. Since the lattice structure changes during transformation, electron paths change and thus the resistivity of the material changes. By measuring the resistance of the metal using a bridge circuit, martensite resistivity has been measured accurately as $100 \mu \Omega \cdot \mathrm{cm}$ and austenite resistivity as $80 \mu \Omega \cdot \mathrm{cm}$ [reference]. With the $20 \%$ change in resistivity, transformation temperatures are easily measured.

Wafer curvature has been measured to research film stresses for many applications including SMA behavior. The curvature $\rho^{-1}$ of a silicon wafer with SMA film on one side is measured by directing a laser at the shiny film surface on the substrate and measuring the reflected angle of the beam. When the laser and photosensor are scanned across the substrate, the slope at each discrete test point resolves the profile of the substrate and the curvature is calculated. Heating and cooling the substrate while taking these measurements every few degrees 
yields a hysteresis plot like the one seen in Figure 2.1. Interpretation of this plot gives transition temperatures, hysteresis width, and the recoverable stress $\sigma_{\text {rec }}$ of the SMA film. The residual stress of the film, corresponding to the martensitic yield stress $\sigma_{m, y}$ [reference], is also found in this manner by taking a measurement at ambient temperature. This curve differs from Figure 1.1 by the downward slope of the film stress with increasing temperature. The film has a higher coefficient of thermal expansion than the substrate and therefore loses stress with increased temperature in its austenite phase. The curve would theoretically go on to intersect the $x$-axis (with zero film stress) at the annealing temperature of the film.

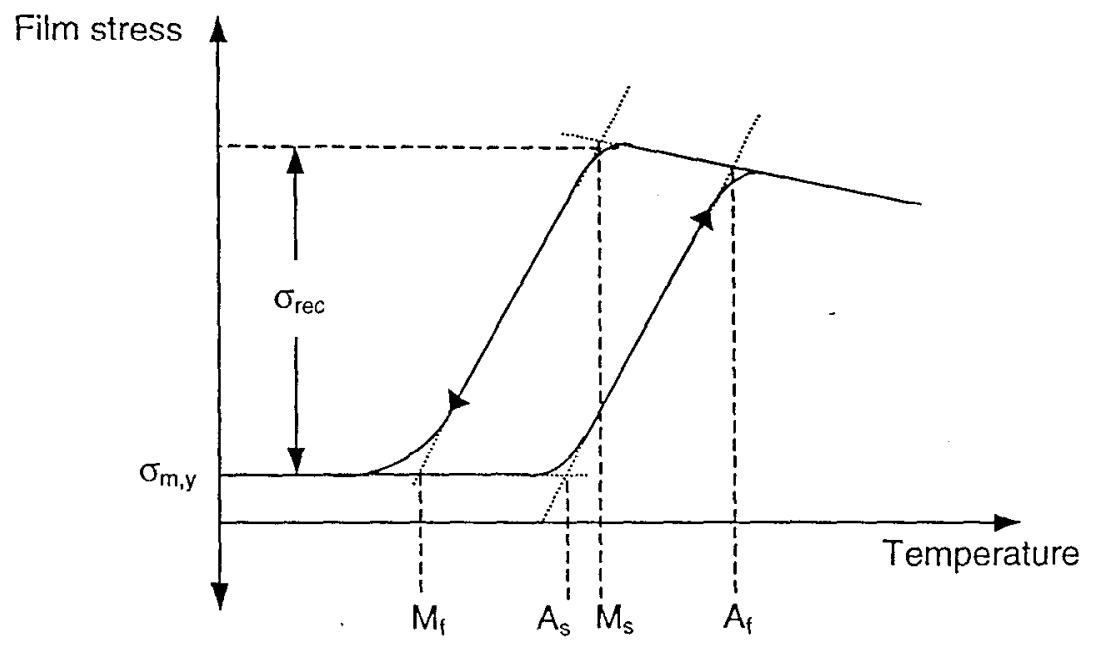

Figure 2.1: Generic hysteresis loop for generated by curvature test with SMA film on silicon wafer substrate. Hysteresis width is given by $A_{f}-M_{s}$ or $A_{s}-M_{f}$.

The film stress for the curve in Figure 2.1 is found by the well known modified Stoney equation [reference], with the radius of curvature $\rho$, the biaxial Young's modulus of the substrate $E_{s} /\left(1-v_{s}\right)$, the substrate thickness $t_{s}$, and the film thickness $t_{f}$ :

$$
\sigma_{f}=\frac{1}{\rho} \frac{E_{s} t_{s}^{2}}{6\left(1-v_{s}\right) t_{f}}
$$

By pressurizing SMA diaphragms and measuring their deformed shape before and after heating, bulge tests are performed. These tests have been done with and without bias pressure during heating, so that shape memory recovery could be determined with and without loading. Results from these studies [references] showed that the deflection of the membrane could be measured by optical profilometry, but the calculation of stress and strain was difficult because of boundary conditions at the edges of a square diaphragm. 
Tunneling electron microscope (TEM) studies are performed on SMAs to investigate microstructure issues like twin formation vs. amorphous residue and precipitate existence. Microscopy is accomplished by creating a XX nm thick sample of the alloy, directing an electron beam at the sample, and measuring the diffraction pattern of the electrons as they go through the alloy. By this method, twin planes are seen as tiger stripes in a highly twinned alloy, while amorphous material has a disorganized pattern and only grain boundaries can be seen. A TEM study can be performed concurrently with any of the testing methods described in this section, but is very expensive, so any microstructural information found from other testing methods is beneficial.

\subsection{New approach for SMA characterization}

\subsubsection{Out-of-plane testing}

In an effort to create a non-destructive testing method for SMA film characterization, the out-of-plane test was developed. Rather than sputtering a film and detaching it from the substrate, a film is sputtered and the substrate is etched out from under it, leaving a freed ligament on which forces can be applied to cause measurable deflections. Figure 2.2 demonstrates the outof-plane method for applying strain to a ligament. By designing the ligaments such that their width is much greater than their thickness and by putting strain relief sections in to control what parts of the ligament experience deformation, bending moments and edge effects could be disregarded. With this test, a force is applied at the center of the ligament, causing pure tensile strain in the two legs of the ligament. The stress and strain are then calculated by the geometry of the ligament and the measured downward deflection.

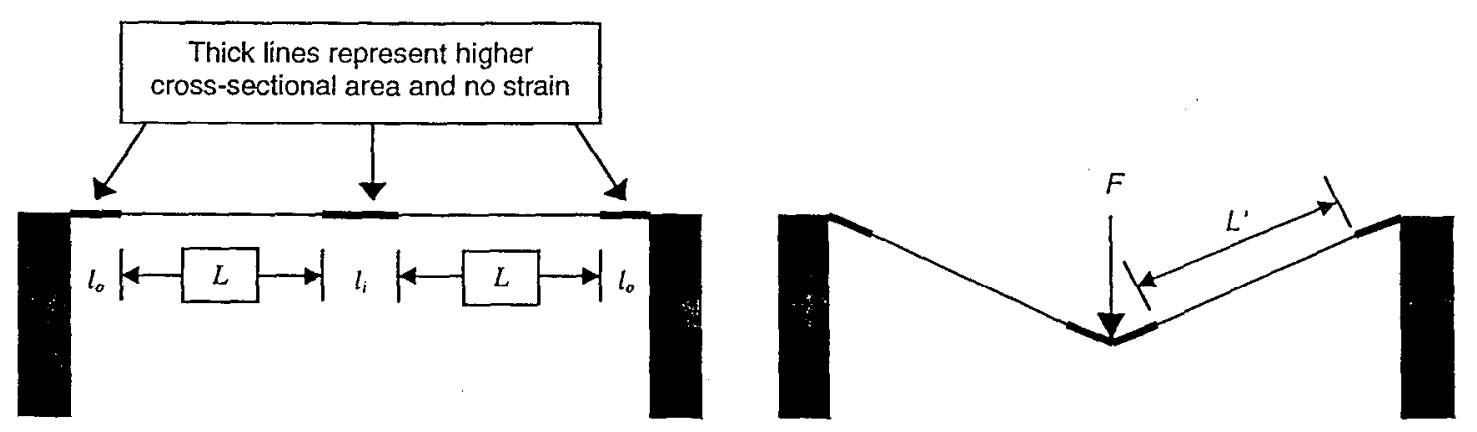

Figure 2.2: Cross-sectional view of a ligament before and after force application during the out-of-plane stretch test. In the absence of a bending moment, the stress in the ligament legs is purely tensile. In this view, $\mathrm{L}$ is the original unstretched length of the ligament leg and $\mathrm{L}^{\prime}$ is the stretched length. The inner strain relief has length $l_{i}$ and the outer reliefs have length $l_{0}$.

This testing method is advantageous because large displacements of the center of the ligament cause small strains in the SMA ligaments. In Miyazaki's tensile tests, the films were 
pulled by the ends and the strain was measurable with the same resolution as the displacement. By translating this large displacement into small strains, lower strains were measurable.

\subsubsection{Differential variable reluctance transduction}

To find the mechanical properties of SMA using the out-of-plane testing concept, the downward displacement and force on the ligament had to be measured. Employing a differential variable reluctance transducer (DVRT) made that task possible. The Microminiature $\odot$ DVRT used in these experiments was purchased from Microstrain, Inc [ref]. Illustrated in Figure 2.3, the DVRT measures small displacements (on the order of $\mu \mathrm{ms}$ ) by differentiating the reluctance output of a magnetic core moving through a coil. When filtered through a demodulator board, the DVRT outputs a voltage proportional to its displacement. In addition to the displacement reading, the force exerted by the DVRT in contact with the ligament is known because the spring constant was calibrated so that knowing the compression of the DVRT spring would result in a highly accurate force measurement.

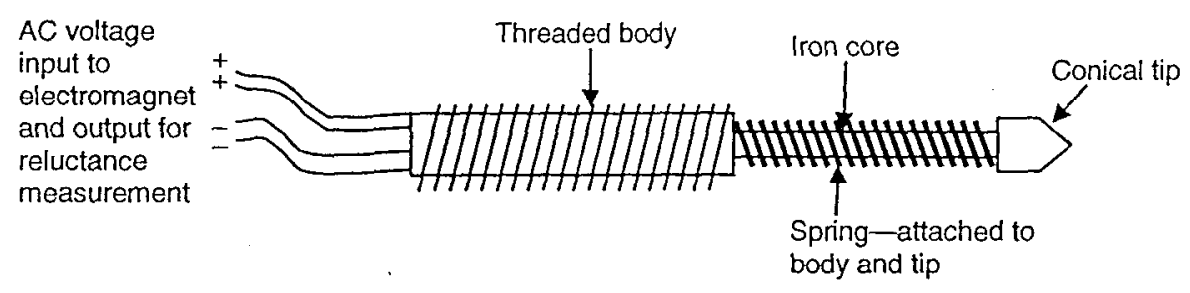

Figure 2.3: Microminiature DVRT, length from wiring to tip is $15 \mathrm{~mm}$.

The DVRT was affixed to a Z-translation stage to allow upward and downward motion controlled by a Mitutoyo Digimatic $₫$ Micrometer Head [reference]. This system was then attached to a second Z-translation stage to permit rough translation to bring the DVRT into proximity of the ligament. The measurement process is outlined in Figure 2.4. Using the DVRT to measure the downward displacement of the center of a ligament involves taking the difference between the downward motion of the micrometer head, to which the DVRT body is attached, and the upward motion of the DVRT head relative to the DVRT body. The first is output on the micrometer's digital readout and the second is output in voltage by the DVRT, so the actual downward displacement of the ligament could be easily found.

Measurements were initiated by positioning the conical DVRT head using the micrometer head until contact was established with the test ligament, as seen in Fig. 2a. At this point, the micrometer head and DVRT output were set to zero. This step in the process added the most uncertainty to the measurement because contact was measured visually through a magnifying lens. To measure the stress-strain characteristics for martensite, the micrometer is 
advanced, compressing the spring and deforming the film. The total displacement of the center of the ligament is the difference between the micrometer reading $d_{m}$ and DVRT reading $d_{1}$ as seen in Fig. 2b. The downward force on the ligament is equal to the product of the spring compression and spring constant $\left(d_{I} \times k\right)$. During SMA actuation, seen in Fig. 2c, the DVRT spring compresses to a value $d_{2}>d_{1}$, exerting greater force on the ligament, equal to $d_{2} \times k$, but causes less strain by decreased deformation of the ligament $\left(d_{m}-d_{2}<d_{m}-d_{1}\right)$.

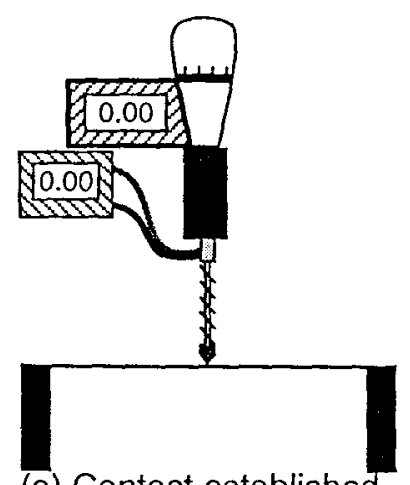

(a) Contact established

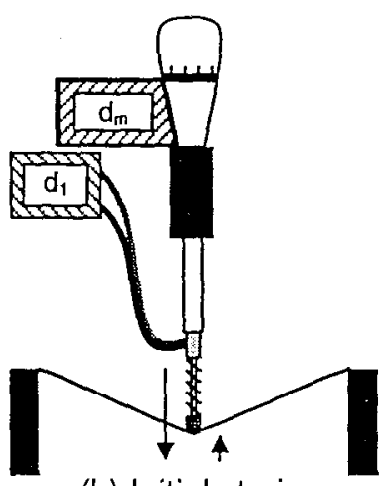

(b) Initial strain

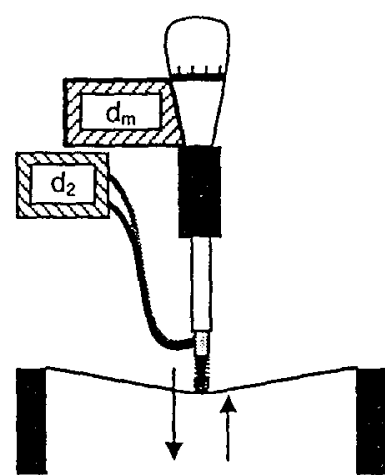

(c) SMA actuation

Figure 2.4: The DVRT head measures displacements and forces of the ligament as the digital micrometer is advanced. The micrometer displacement and the DVRT compression are displayed on digital readouts during each step of the measurement. Resistive heating of the ligament causes actuation of the SMA.

\subsubsection{Resistive heating}

Many MEMS devices rely on resistive heating of some kind to achieve actuation in one

form or another. With the NiTiCu ligaments, resistive heating, or Joule heating, was accomplished by simply passing a current through the ligament until full transformation took place. Joule's law (Equation 2-2) gives the amount of heat flow $Q$ generated by a current $I$ through a resistor with resistance $\mathrm{R}$ for a time $t$.

$$
Q=I^{2} R t
$$

Since the time derivative of heat flow is directly proportional to the change in temperature by Newton's law of conduction, when convective losses are neglected, the change in temperature $\Delta T$ of the ligament can be found with respect to the driving current. The following equations contain the thermal conductivity $K$ of the SMA, the thickness $h$ of the ligament, the cross-sectional area $A_{c}$, the surface area $A_{s}$, the ligament resistivity $\rho$, and the distance between the electrical contacts $L_{e}$. 


$$
\begin{aligned}
\Delta T & =\dot{Q} \frac{h}{K A_{s}} \\
& =I^{2} R \frac{h}{K A_{s}} \\
& =I^{2} \frac{\rho L_{e} h}{K A_{c} A_{s}}
\end{aligned}
$$

When resistive heating drives the ligament temperature above the austenite finish temperature of the alloy, actuation occurs, compressing the DVRT spring to the state seen in Figure 2.4(c).

\subsubsection{Ligament geometry}

Freestanding NiTiCu ligaments were created for use in the out-of-plane stretch tests described in this paper. The geometry of these ligaments is seen in Figure 2.5 with the shaded area marking the NiTiCu film and the cross-hatched area indicating the underlying silicon substrate.
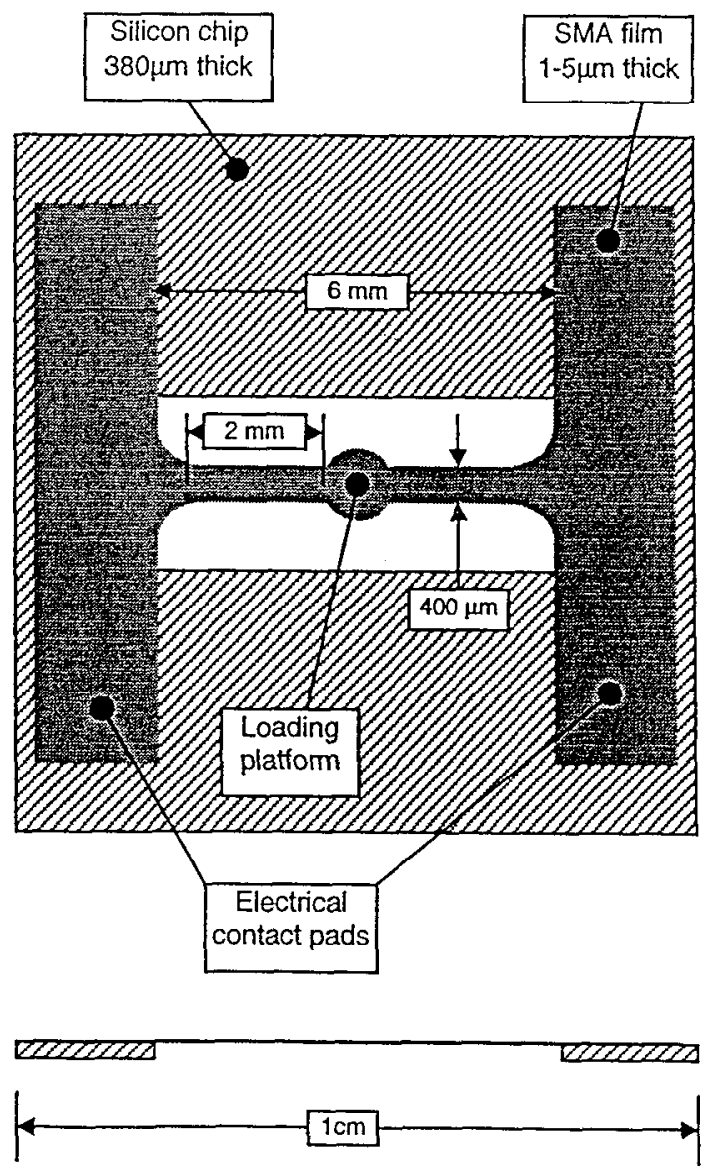

Figure 2.5: Geometry of a ligament test chip. Gray areas are SMA film and cross-hatched areas are silicon. 
The $1 \mathrm{~cm}$ square test chips were designed so that they could slide into place beneath the DVRT head to line up the center of the ligament with the conical point of the DVRT. Spring clips hold down the test chips and also act as the electrical contacts to drive current through the ligament. The NiTiCu was patterned to include $1.9 \mathrm{~mm} \times 7 \mathrm{~mm}$ contact strips on each side of the ligament where the chip is held down by the spring clips. One-half millimeter wide strain reliefs lead from the contact pads to the ligament legs, which are $2 \mathrm{~mm}$ long and $400 \mu \mathrm{m}$ wide each. The loading platform, a $1 \mathrm{~mm}$ diameter circle with rounded strain reliefs on each side, connects the two ligament legs. When a ligament is loaded in the center of this platform and deflected downward, the only part of the ligament to undergo strain is the $2 \mathrm{~mm}$ leg sections.

\subsection{Experimental setup}

\subsubsection{Actuation testing}

To obtain actuation data for the NiTiCu films, ligaments underwent testing using the DVRT testing instrument discussed in this section and Appendix B. These tests are outlined as follows.

1. To get an idea of the power required for ligament actuation, the resistance of each ligament was measured with a digital multimeter by probing on each of the electrical contact pads on either side of the ligament.

2. A ligament was slid onto the aluminum base and under the conductive clips. Nylon washers insulated clips from the base and the silicon chip insulated the ligaments.

3. A direct current power source was connected to the conductive clips and a $1.0 \Omega$ precision resistor was placed in parallel with the resistor. The current from the DC power source had a precision of only $0.1 \mathrm{~A}$, so the precision resistor in parallel was necessary to bring the current precision up to $0.01 \mathrm{~A}$.

4. The ligament was aligned with the DVRT so that the conical tip was centered in the middle of the ligament loading platform.

5. The precision micrometer was advanced until in proximity of the ligament and current through the ligament was applied. Current was increased until the ligament heated enough to recover its residual stress and pull tight across the window. The micrometer was then advanced again until contact was made between the conical tip and the loading platform. This process is seen in the first two steps of Figure 2.6.

6. The micrometer was advanced $300 \mu \mathrm{m}$ to provide an initial load on the ligament.

7. Current was applied to the ligament and increased until deflection was at its maximum. 
8. The micrometer was backed off of the ligament and the current found in Step 7 was applied to the ligament. At this point, the second step of Figure 2.6 was repeated to zero the DVRT and current was removed from the ligament.

9. The micrometer was advanced to $100 \mu \mathrm{m}\left(d_{m}\right)$.

10. A measurement of DVRT displacement $\left(d_{1}\right)$ was taken, current was applied, and a second measure of DVRT displacement $\left(d_{2}\right)$ was taken.

11. Step 10 was repeated as the micrometer was advanced in $50 \mu \mathrm{m}$ increments to $d_{m}=1000 \mu \mathrm{m}$ and every $100 \mu \mathrm{m}$ thereafter until fracture of the ligament.
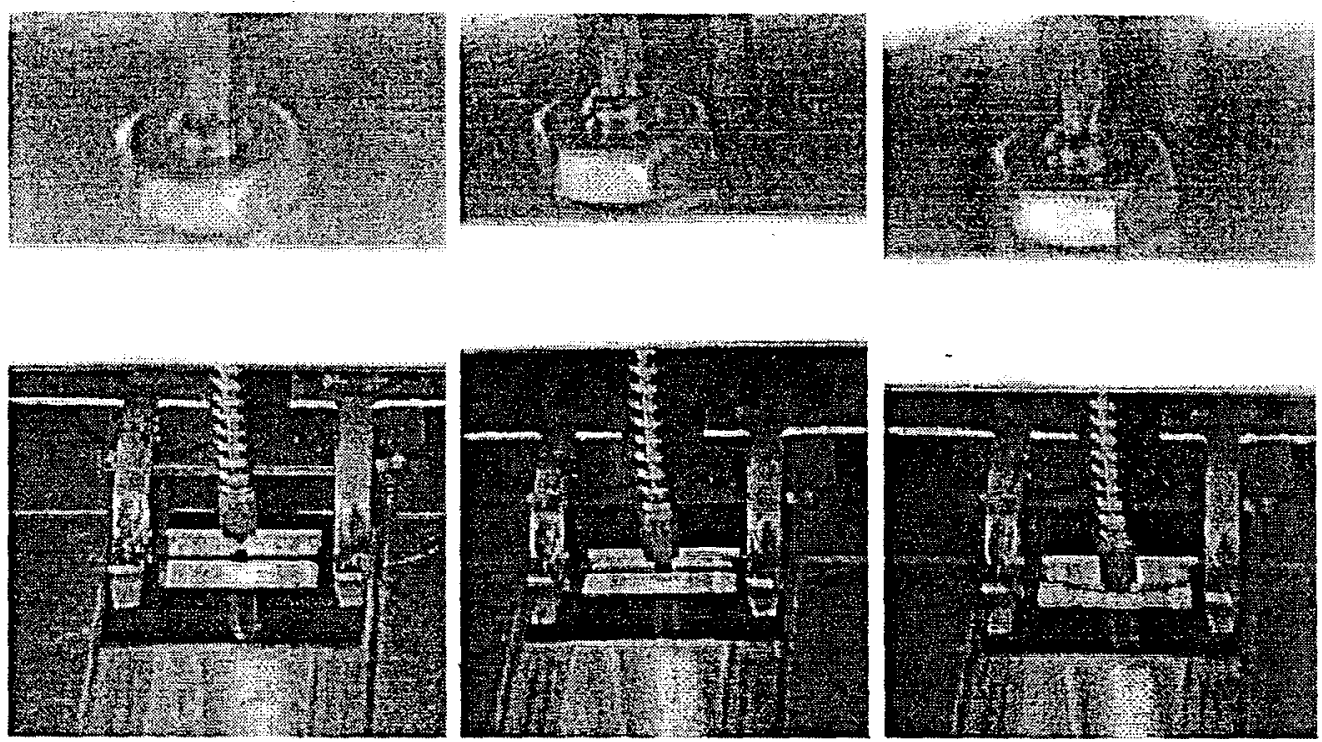

Figure 2.6: Photographs of the process of bringing the DVRT into proximity of the ligament, making contact, and loading, causing displacement.

\subsubsection{Static testing}

In order to determine the stress-strain reaction to loading in each phase, separate tests were performed in the martensite and austenite phase. Martensite testing proceeded by following Steps 1-5 from the previous section, then loading to take a measurement every $50 \mu \mathrm{m}$ from $100-$ $1000 \mu \mathrm{m}$ and every $100 \mu \mathrm{m}$ thereafter until fracture. No current was applied during the loading process, so that all measurements were taken at room temperature while the alloy was still in its martensite phase. In austenite tests, Steps 1-8 from the last section were followed to find the necessary power to fully transform the alloy to austenite and to zero the DVRT. Then the current found in Step 7 was applied to the ligament and loading took place in the same manner as in martensite tests. Through this method, austenite and martensite stress-strain curves were obtained separately and without the affects of actuation cycling. 


\subsubsection{Fatigue testing}

To create the cyclic power needed for fatigue testing, a function generator was connected through a power amplifier to the ligament's electrical contact pads. Repetition of Steps 1,2,4 and 5 from Section 2.4.1 allowed alignment of the DVRT with the test ligament. The power supply setup is shown in Figure 2.7, with indications of probe locations for digital acquisition. The function generator drove a square wave signal through the ligament, with adjustable duty cycle to account for the difference in time to heat a ligament with electrical power and time to cool it by passive convection. Signals of the DVRT output, the voltage through the precision resistor, and the voltage through the ligament were acquired through a DAQ card by National Instruments' Labview virtual interface. The DVRT output was input on Channel 1 of the DAQ to indicate displacements of the ligament during cycling. The voltage through the ligament was the input of Channel 2 and was displayed in real time to help adjust input voltage so that shape memory recovery and cycling frequency were maximized. A precision resistor was placed in series with the ligament and the voltage was read through Channel 3. When this voltage was divided by the pre-measured resistance of the precision resistor, the current through the ligament was found. Then, when the voltage through the ligament was divided by the current through the resistor and the ligament in real time, the simultaneous measurements of the alloy's resistance and the stressstrain behavior were made.

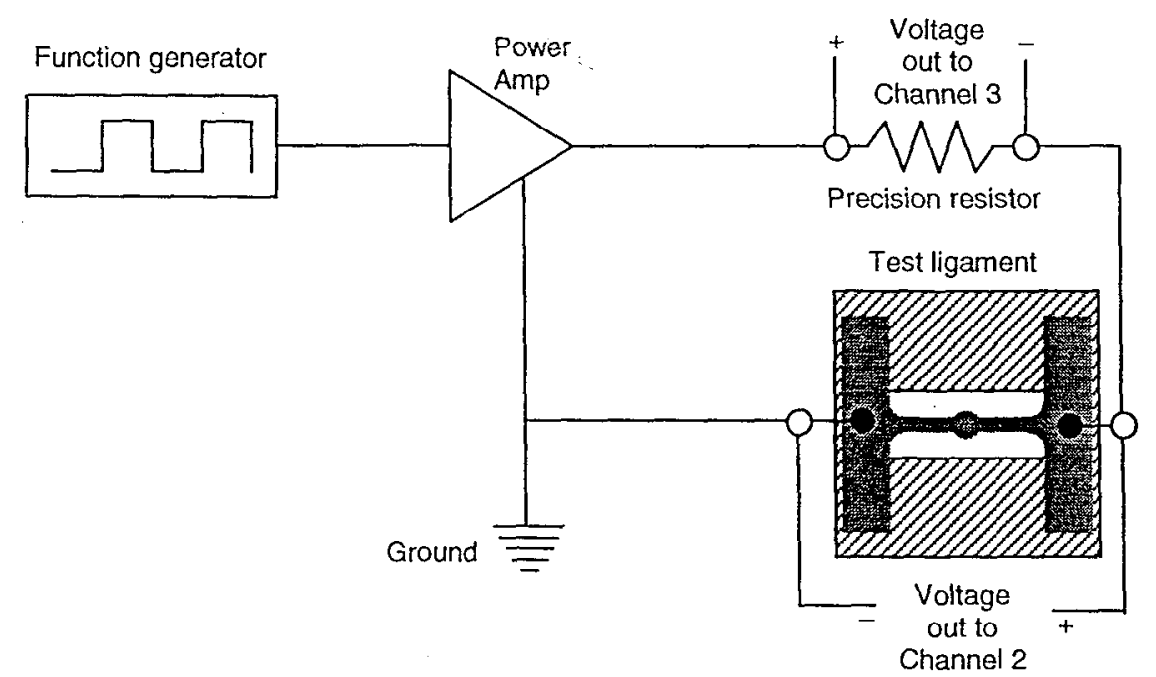

Figure 2.7: Schematic of ligament power supply during fatigue measurements and probe locations for digital data acquisition. 


\subsection{Stress-strain theory for SMA}

\subsubsection{Engineering strain}

The engineering strain is the most common strain calculation method and was used as a first pass to determine the strain of the material. It measures the ratio of the change in length divided the original length of a tensile specimen. It is a good approximation of the actual strain for small deformations, but when large deformations are encountered, the true strain is the better measurement. Here, the engineering strain is presented as a basis of comparison and to show the difference in strain calculation techniques when large strains are encountered.

\subsubsection{Residual strain $\varepsilon_{o}$}

To fully understand all of the forces acting upon these ligaments, the residual strain $\varepsilon_{o}$ must first be determined. This strain exists in the ligaments because they have cooled and contracted from their annealed states, taking on a residual tensile stress. This is the only destructive test on these ligaments and therefore was only performed on a few chips out of every wafer.

A ligament starts off with each leg of length $L$ under some residual strain. When the ligament is broken and heated to reset to the annealed shape, a finite gap width $e$ was measured between the severed ends and the length of each leg of the ligament was measured as $L_{o}$. The residual strain was therefore determined as follows:

$$
\begin{aligned}
& 2 L=2 L_{0}+e \\
& \varepsilon_{0}=\frac{2 L-2 L_{0}}{2 L_{0}}=\frac{e}{2 L-e}
\end{aligned}
$$

\subsubsection{Initial strain $\varepsilon_{I}$}

As a load is applied to the ligament, the micrometer is advanced a distance $d_{m}$ and the DVRT spring compresses a distance $d_{l}$. For simplicity, make the substitution

$$
d=d_{m}-d_{1}
$$

where $d$ is the total downward displacement of the center of the ligament. Referring to Figure 2.2 , the original length $L_{\text {orig }}$ of each leg of the ligament is given by

$$
L_{\text {orig }}=\frac{l_{i}}{2}+l_{o}+L
$$


and the final length $L_{\text {final }}$ is given by

$$
L_{\text {final }}=\frac{l_{i}}{2}+l_{o}+L^{\prime}
$$

With the help of Pythagoras, the initial strain is found:

$$
\begin{aligned}
d^{2}+L_{\text {orig }}^{2} & =L_{\text {final }}^{2} \\
d^{2}+\left(\frac{l_{i}}{2}+l_{o}+L\right)^{2} & =\left(\frac{l_{i}}{2}+l_{o}+L^{\prime}\right)^{2} \\
d^{2}+L\left(l_{i}+2 l_{o}+L\right) & =L^{\prime}\left(l_{i}+2 l_{o}+L^{\prime}\right) \\
L^{\prime} & =\sqrt{d^{2}+\left(\frac{l_{i}}{2}+l_{o}+L\right)^{2}}-\frac{l_{i}}{2}-l_{o} \\
\varepsilon_{1} & =\frac{L^{\prime}-L}{L}+\varepsilon_{0} \\
\varepsilon_{1} & =\frac{\sqrt{\left(d_{m}-d_{1}\right)^{2}+\left(\frac{l_{i}}{2}+l_{o}+L\right)^{2}}-\left(\frac{l_{i}}{2}+l_{o}+L\right)}{L}+\varepsilon_{0}
\end{aligned}
$$

\subsubsection{Actuated strain $\varepsilon_{2}$}

After actuation of the ligament, the DVRT head compresses more, giving a new deflection $d_{2}$. By substituting this for $d_{1}$ in the above equation, the new strain $\varepsilon_{2}$ is found:

$$
\varepsilon_{2}=\frac{\sqrt{\left(d_{m}-d_{2}\right)^{2}+\left(\frac{l_{i}}{2}+l_{o}+L\right)^{2}}-\left(\frac{l_{i}}{2}+l_{o}+L\right)}{L}+\varepsilon_{0}
$$

\subsubsection{Recoverable strain $\varepsilon_{\text {rec }}$ and percentage of strain recovered $\varepsilon_{\%}$}

Once the initial strain and actuated strain are known, the recoverable strain is simply the difference between them and is defined as the amount of strain that the ligament can recover at a given preload. This is better seen by the percent of strain recovered, showing the proportion of strain recovered versus initial strain.

$$
\begin{aligned}
\varepsilon_{r e c} & =\varepsilon_{1}-\varepsilon_{2} . \\
\varepsilon_{\%} & =\frac{\varepsilon_{r e c}}{\varepsilon_{1}}
\end{aligned}
$$




\subsubsection{True strain}

The true strain differs from the engineering strain by calculating the instantaneous defornation and integrating from the initial to the final deformed state. First, a differential strain element $d \varepsilon$ is presented. It is the ratio of instantaneous length, $d l$ to prior length, $l$. With that in mind, the total strain during deformation is the integral of $d \varepsilon$ from the original length $l_{o}$ to the final deformed length $l$.

$$
\begin{aligned}
d \varepsilon & =\frac{d l}{l} \\
\varepsilon & =\int_{l_{0}}^{l} \frac{d l}{l}=\ln \left(\frac{l}{l_{0}}\right)
\end{aligned}
$$

Using the true strain, the residual, initial, and actuated strain are reevaluated below. The recoverable strain and percent recoverable strain equations, however, remain the same.

\subsubsection{Initial length $l_{o}$}

In the case of true strain, the residual strain $\varepsilon_{0}$ is not considered, but the gap width $e$ is very important in determining the original length $l_{o}$ of the specimen. To find the $l_{o}$, half of the gap width must be subtracted from each side of the ligament, leading to the following:

$$
\begin{aligned}
& l_{o}=L-\frac{e}{2}=L-\frac{e L}{2 L}=L\left(1-\frac{e}{2 L}\right) \\
& l_{o}=L\left(\frac{2 L-e}{2 L}\right)
\end{aligned}
$$

where $L$ is the length of each ligament leg.

\subsubsection{Initial strain $\varepsilon_{I}$}

With $l_{o}$ determined, the final length $l$ is all that is needed to determine the induced in the ligament. To find this value, the variable $d$ is used again as the downward deflection of the center of the ligament, along with the angle $\theta$ between the deformed ligament leg and the horizontal. The value $L_{\text {rigid }}$ appears here as the length of the section of ligament where negligible strain occurs, but in the test geometry, the cancels because it is simply equal to one half the length of the strained part of the ligament. This definition yields the following: 


$$
\begin{aligned}
\tan \theta & =\frac{d}{L+L_{r i g i d}}=\frac{d}{L+\frac{L}{2}}=\frac{2 d}{3 L} \\
\theta & =\tan ^{-1}\left(\frac{2 d}{3 L}\right)
\end{aligned}
$$

At this point, $l$ can be determined:

$$
\begin{aligned}
\frac{L+L_{\text {rigid }}}{l+L_{\text {rigid }}} & =\cos \theta=\frac{\frac{3}{2} L}{l+\frac{1}{2} L} \\
l & =\frac{3 L}{2 \cos \theta}-\frac{L}{2} \\
l & =\frac{3 L}{2 \cos \left[\tan ^{-1}\left(\frac{2 d}{3 L}\right)\right]}-\frac{L}{2} .
\end{aligned}
$$

Therefore, the initial strain can be found and the trigonometric functions can be simplified by their definitions:

$$
\begin{aligned}
& \varepsilon_{1}=\ln \left[\frac{\left.\frac{3 L}{2 \cos \left[\tan ^{-1}\left(\frac{2\left(d_{m}-d_{1}\right)}{3 L}\right)\right]}-\frac{L}{2}\right)}{L\left(\frac{2 L-e}{2 L}\right)}\right] \\
& \varepsilon_{1}=\ln \left[\frac{2 L}{2 L-e}\left(\frac{3}{2} \sec \left\{\tan ^{-1}\left(\frac{2\left(d_{m}-d_{1}\right)}{3 L}\right)\right\}-\frac{1}{2}\right)\right] \\
& \varepsilon_{1}=\ln \left[\frac{2 L}{2 L-e}\left(\frac{\sqrt{4\left(d_{m}-d_{1}\right)^{2}+9 L^{2}}-L}{2 L}\right)\right] \\
& \varepsilon_{1}=\ln \left[\frac{\sqrt{4\left(d_{m}-d_{1}\right)^{2}+9 L^{2}}-L}{2 L-e}\right)
\end{aligned}
$$




\subsubsection{Actuated strain $\varepsilon_{2}$}

Through the same method as finding the initial strain, substituting $d_{2}$ for $d_{1}$, the actuated strain is found:

$$
\varepsilon_{2}=\ln \left(\frac{\sqrt{4\left(d_{m}-d_{2}\right)^{2}+9 L^{2}}-L}{2 L-e}\right)
$$

\subsubsection{Stress}

To find the recoverable stress of the SMA in the ligaments, the difference is taken between the initial stress and the final stress. This value is the amount of stress added to the ligament due to the phase change and therefore characterizes the amount of force that a ligament with any given cross-sectional area can produce. For either the initial or the final state, the force in the ligament can be found by resolving the force in the spring into its normal and tensile components with respect to the ligament. This relationship is shown in the following equation, where $F_{\text {lig }}$ is the tension of the ligament and $F_{k}$ is the reactive force of the spring's compression.

$$
F_{\text {lig }}=\frac{F_{k}}{\sin \theta}
$$

\subsubsection{Residual stress $\sigma_{0}$}

To find the residual stress in the ligament, the biaxial residual stress that was measured from a blank curvature specimen and translated to a uniaxial stress by translating from twodimensional to one-dimensional stress. In the following, $v$ is the Poisson's ratio of bulk NiTiCu.

$$
\sigma_{0}=(1-v) \sigma_{0(\text { biaxial })}
$$

\subsubsection{Initial stress $\sigma_{1}$}

In the initial stress-state, the force in the spring is equal to the spring constant $k$ multiplied by the initial DVRT reading $d_{l}$. By dividing this force by the cross sectional area (where $w=$ width and $h=$ height of ligament) and substituting the appropriate geometrical constraints for $\theta$, the initial stress is found. 


$$
\begin{aligned}
F_{k} & =k d_{1} \\
F_{\text {lig }} & =\frac{k d_{1}}{\sin \theta} \\
\sigma_{1} & =\frac{F_{l i g}}{A}=\frac{k d_{1}}{w h \sin \left[\tan ^{-1}\left(\frac{2\left(d_{m}-d_{1}\right)}{3 L}\right)\right]} \\
\sigma_{1} & =\frac{k d_{1}}{w h} \csc \left[\tan ^{-1}\left(\frac{2\left(d_{m}-d_{1}\right)}{3 L}\right)\right] \\
\sigma_{1} & =\frac{k d_{1} \sqrt{4\left(d_{m}-d_{1}\right)^{2}+9 L^{2}}}{2 w h\left(d_{m}-d_{1}\right)}
\end{aligned}
$$

It is important to notice that the stress in the film is found simply from the tension in the ligament, resolved from its reaction force against the ligament. Therefore, no residual stress term is included in the initial or final stress equations.

\subsubsection{Final stress $\sigma_{2}$}

Similarly, by substituting $d_{2}$ for $d_{l}, \sigma_{2}$ is determined.

$$
\sigma_{2}=\frac{k d_{2} \sqrt{4\left(d_{m}-d_{2}\right)^{2}+9 L^{2}}}{2 w h\left(d_{m}-d_{2}\right)}
$$

\subsubsection{Recoverable stress $\sigma_{\text {rec }}$}

The recoverable stress is a measure of how much stress is added to the ligament during actuation. It is therefore a simple difference between the initial and final stress:

$$
\sigma_{\text {rec }}=\sigma_{2}-\sigma_{1}
$$

\subsubsection{Work output}

To understand the achievable work output of the ligament, the equation

$$
\begin{aligned}
d W & =F d x \\
W & =\int_{d_{1}}^{d_{2}} F d x
\end{aligned}
$$

is utilized, where $W$ is work output, $F$ is the spring force that the ligament acts against, and $d s$ is the change in linear position with the endpoints given by $d_{I}$ and $d_{2}$, the displacement readings from the DVRT. This will measure the work done by compressing the DVRT spring as the 
ligament actuates. It is seen in Figure 2.8 that this integral is simply the area under the forcedisplacement curve of the spring between the two offsets.

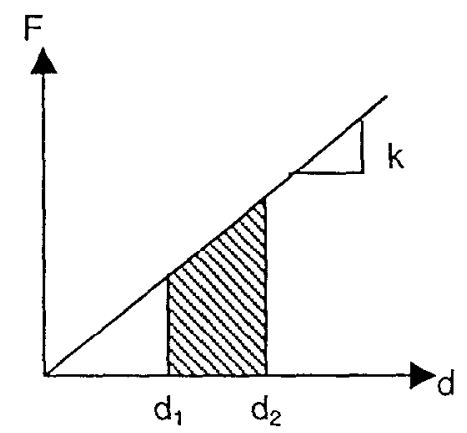

Figure 2.8: The work output of the ligament is the shaded area under the force-displacement curve.

Using Hooke's law to substitute for $F$, the integral is solved and the work output is found:

$$
\begin{aligned}
F & =k x \\
W & =\int_{d_{1}}^{d_{2}} k x d x \\
W & =\frac{k}{2}\left(d_{2}^{2}-d_{1}^{2}\right)
\end{aligned}
$$

\subsection{Expected results}

\subsubsection{Stress-strain curves}

Shape memory alloys behave differently than other materials because of their two-phase nature. Figure 2.9 depicts the theoretical stress-strain behavior of shape memory alloys in their martensite and austenite phases. The lower curve on this plot is the expected martensite behavior. It follows a slope upward until the stress for twin movement is encountered. The plot then becomes horizontal as the twins move with respect to each other and the alloy experiences pure strain with no additional stress. When twins cannot move any more, the material should experience a linear relation between stress and strain until the yield stress is encountered, at which plastic yielding begins. The ratio of stress to strain decreases as dislocations begin to move and finally, the ultimate tensile stress is reached, at which point fracture of the alloy occurs. The anticipated austenite curve is less abnormal because of the lack of twins. There is a linear relationship between stress and strain until the yield stress is reached; at which point the slope decreases. At the ultimate tensile stress, the curve ends due to fracture in the ligament. 


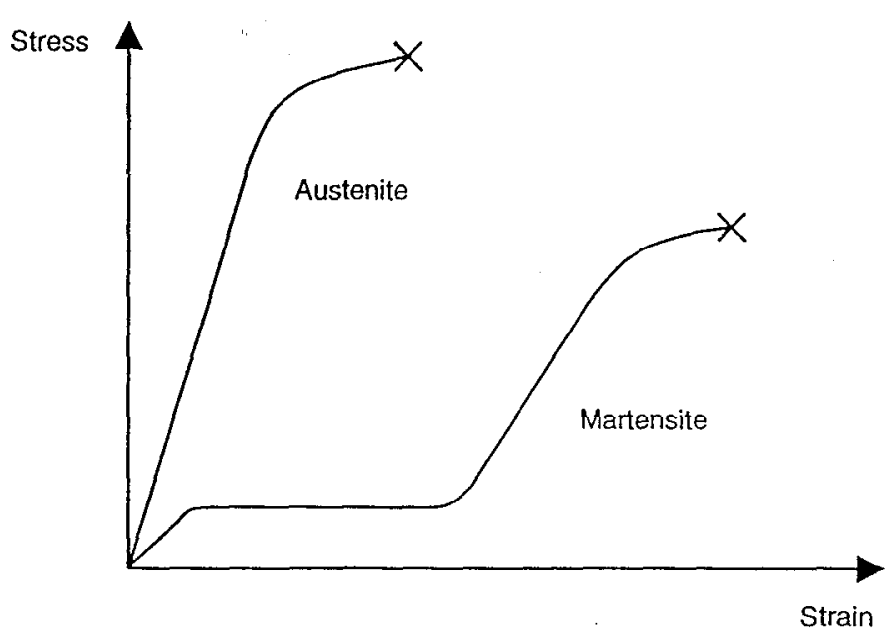

Figure 2.9: Theoretical stress-strain curve for shape memory alloys. Shape memory actuation occurs by heating from martensite to austenite, thereby removing strain for a given stress. Ultimate tensile stress is indicated by X's at the end of each theoretical plot.

\subsubsection{Recoverable stress and strain}

By examining Figure 2.9, the recoverable stress and strain can be found depending on operating conditions. Actuation of a ligament causes a shift from the operating point on the martensite curve to a point on the austenite curve. The geometry of the ligament and the linear spring involved in this experiment cause the stress and strain to change in this process. If the same experiment was performed with stress or strain held constant during actuation, the movement from one curve to the other would be purely horizontal or vertical, respectively. For the purposes of this experiment, imagining negatively sloped diagonal lines that intersect the martensite and austenite curves in as shown in Figure 2.10 helps to elucidate the recoverable stress and strain behavior of the ligaments.

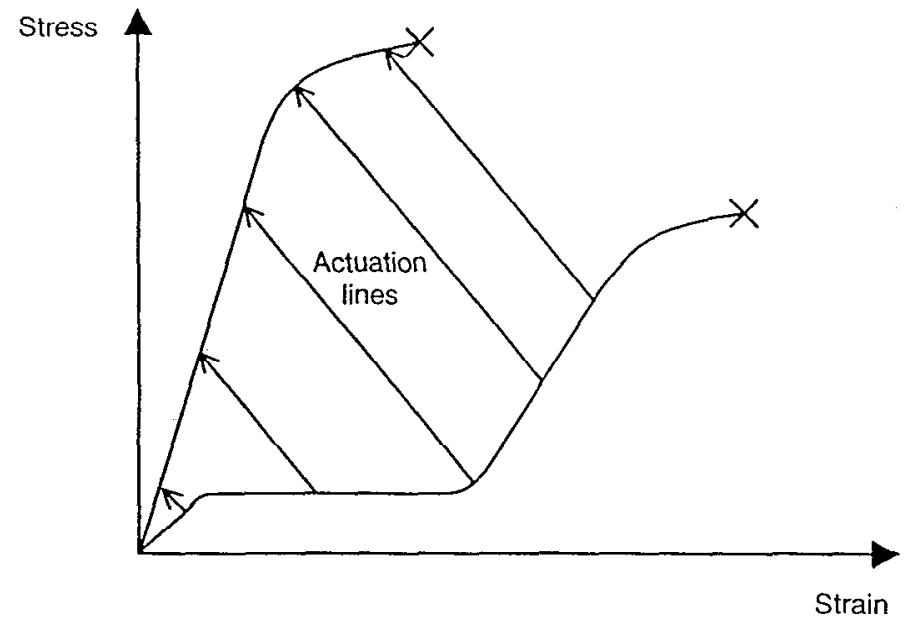

Figure 2.10: Actuation of a ligament causes a shift from an operating point on the martensite curve to a point on the austenite curve. During this process, both stress and strain change due to the conditions of this experiment. 
The expected recoverable stress and strain for this situation are the vertical and horizontal components of the actuation lines of Figure 2.10. When plotted versus the initial strain (i.e. the martensite strain), the theoretical curves of Figure 2.11 are generated.

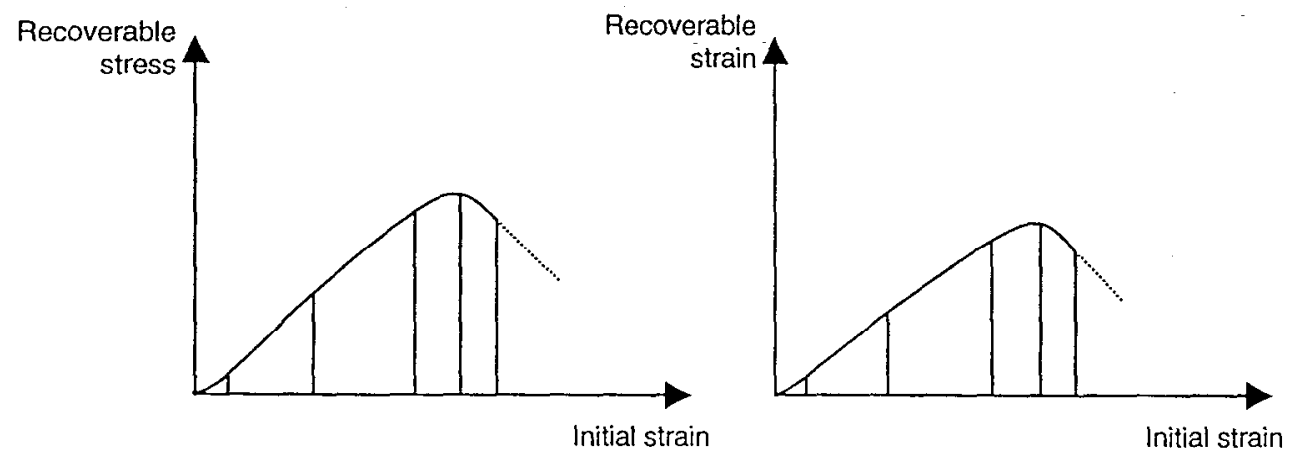

Figure 2.11: Theoretical recoverable stress and recoverable strain $v$ s. initial strain. The recoverable stress and strain peak at a certain initial strain and then fall off with austenite plastic yielding.

\subsubsection{Changes in resistivity}

With the change in microstructure, atom spacing and total volume of the shape memory alloy changes. Accompanying this change is a change in resistivity of the alloy. The reported resistivity of martensite is $100 \mu \Omega \cdot \mathrm{cm}$, while austenite has a resistivity of $80 \mu \Omega \cdot \mathrm{cm}$. This change in resistivity is detectable using the out-of-plane tester by measuring the resistance of the ligament with and without heating. The resistance of the ligaments was expected to decrease by roughly $20 \%$ upon entering austenite phase, with some slight differences due to the change in length and volume associated with the phase change. The relationship between resistance $R$, resistivity $\rho$, length $L$, and cross-sectional area $A$ of the resistive medium is seen in the following equation.

$$
R=\frac{\rho L}{A}
$$

\subsection{In-line testing capabilities}

Not limited to characterizing thin films, the instrument described in this paper can also be utilized to perform in-line qualification of microactuators. For example, assume that a microactuator uses a piezoelectric bimorph cantilever as its mechanism of motion. When a voltage is applied across this bimorph, the top piezoelectric layer compresses and the bottom layer expands, creating a bending moment in the cantilever. By adding a point load at the tip of this cantilever with the DVRT head, then applying a voltage to the bimorph, the force-deflection data can be obtained for the system. Varying voltage across the bimorph produces additional 
curves, and voltage set points for specified actuation can be determined for each wafer of actuators, thereby qualifying their behavior.

A wafer of freestanding shape memory actuators could also be qualified using this

system. This is a powerful tool because of the varying shape memory behavior experienced with different film deposition and processing conditions. 


\section{Chapter 3: Micromachining the ligaments}

\subsection{Introduction}

In order to utilize the simplicity of the out-of-plane method of testing ligaments, freestanding films had to be created by etching windows from beneath them. Many attempts were made to produce these structures before success was found. By building on previous knowledge of processing parameters and inventing other processing methods, ligaments with the geometry seen in Figure 2.5 were created. The processing diagram is seen in Figure 3.1 and explained in the following sections.

\begin{tabular}{|c|c|c|c|}
\hline $\begin{array}{l}1 . \\
2 .\end{array}$ & $\begin{array}{l}\text { Bare silicon wafer } \\
\text { Deposit oxide onto wafer }\end{array}$ & & $\begin{array}{l}\text { Etch oxide from exposed backside area } \\
\text { in buffered }(6: 1) \mathrm{HF}\end{array}$ \\
\hline 3. & Deposit NiTiCu onto one side of wafer & 9. & DRIE through Si wafer in STS etcher \\
\hline 4. & Spin AZ1518 onto NiTiCu and pattern & 10. & Remove oxide membranes in Technics \\
\hline 5. & Etch NiTiCu in 20:20:1 $\mathrm{HCl}: \mathrm{HNO}_{3}: \mathrm{HF}$ & & RIE etcher \\
\hline $\begin{array}{l}6 . \\
7\end{array}$ & Spin AZ4620 onto backside and pattern & 11. & Soak in acctone to remove carrier wafer \\
\hline 7. & $\begin{array}{l}\text { Bond SMA side to carrier wafer with } \\
\text { AZ1518 }\end{array}$ & & \\
\hline
\end{tabular}
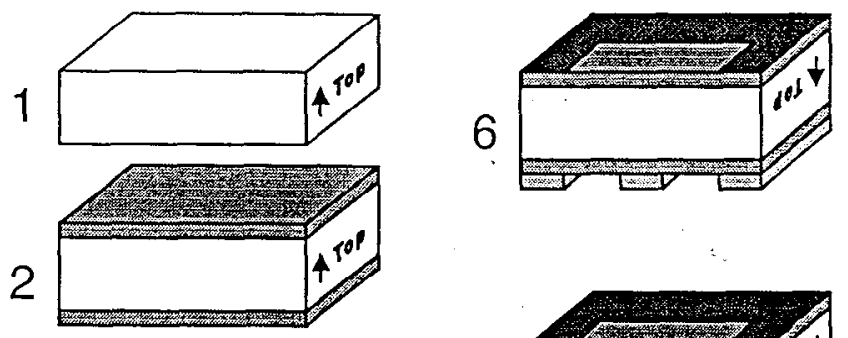

9
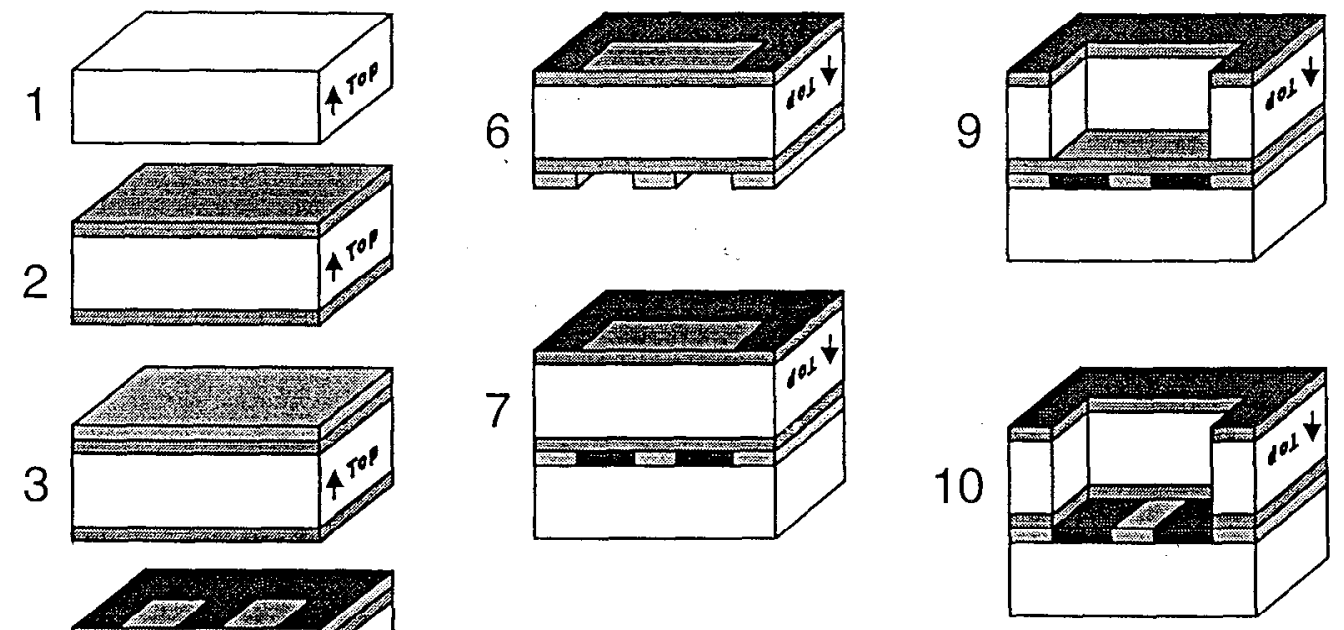

4
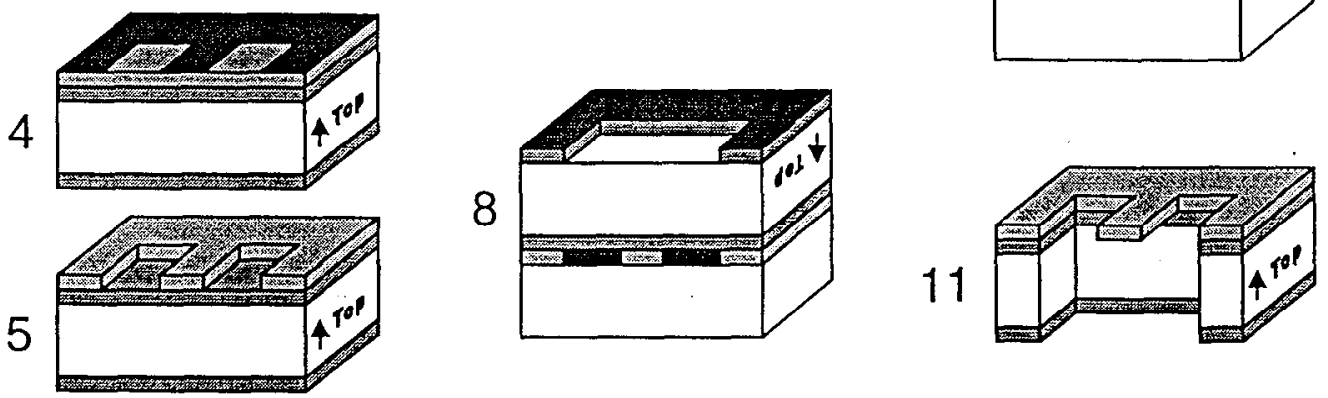

Figure 3.1: Successful processing procedure for the creation of freestanding SMA ligaments. 


\subsection{Successful processing procedure}

\subsubsection{Thermal $\mathrm{SiO}_{2}$ coating of silicon wafers}

Four inch diameter, $380 \mu \mathrm{m}$ thick $\{100\}$ Si wafers were RCA cleaned and inserted into a quartz furnace tube for the purpose of silicon dioxide $\left(\mathrm{SiO}_{2}\right)$ growth. The furnace was heated to $100{ }^{\circ} \mathrm{C}$ while being flushed with nitrogen. When the target temperature was reached, dry oxygen was input into the furnace, leading to dry $\mathrm{SiO}_{2}$ growth, for five minutes. Steam was then pumped into the furnace, leading to faster growth of $\mathrm{SiO}_{2}$ for two hours. Oxide growth was completed by five more minutes of dry oxidation. The dry oxide layers provided 1) a strong bond between the Si wafers and the faster grown wet oxide and 2) a stable outer layer on top of the wet grown oxide to prevent further surface reactions. This deposition procedure led to $0.6 \mu \mathrm{m}$ of $\mathrm{SiO}_{2}$, which was measured by a Nanospcc AFT ??? [refercncc], which measures the thickness of refractive layers by refractive index differences between the $\mathrm{SiO}_{2}$ and the $\mathrm{Si}$.

This $\mathrm{SiO}_{2}$ layer was used to protect the wafer during wet chemical etching and to protect the SMA during plasma etching. Section 3.3.3 confronts the problems of not using this layer for protection.

\subsubsection{SMA deposition onto silicon wafers}

Nickel-titanium-copper films were DC magnetron sputter-deposited at $150 \mathrm{~W}$ from a 33 $\mathrm{mm}$ diameter NiTiCu target while heating the substrate. Films were deposited in a high vacuum chamber under $5.4 \times 10^{-8}$ to $1.9 \times 10^{-7}$ Torr base pressure in $8 \mathrm{~m}$ Torr argon. Thermocouples mounted to the $\mathrm{Si}$ substrate surface indicated substrate temperatures of $510^{\circ} \mathrm{C}$ at deposition start and $540^{\circ} \mathrm{C}$ upon termination. A deposition time of 2 hours resulted in average film thickness across a wafer of $1.9 \mu \mathrm{m}$ with variations of $\pm 0.4 \mu \mathrm{m}$. Thickness variation (seen in Figure 3.2) was due to the low target to substrate diameter ratio, resulting in the thickest film depositing directly in line with the target and diminished film thickness from the center to the edge of the wafer. Film composition, measured by electron microprobe analysis, was 39.5 at $\% \mathrm{Ni}$, 52at\% $\mathrm{Ti}$, and $8.5 \mathrm{at} \% \mathrm{Cu}$.

Copper was used as an alloying agent because it has empirically led to more stable shape memory effects and lower hysteresis widths [reference]. When nickel and titanium are used without copper to produce shape memory effects, small changes in composition make large differences in transformation temperatures, recoverable stresses, and the lattice structure of the different phases. For instance, some compositional configurations lead to an R-phase transformation upon cooling from austenite to martensite. Since the R-phase has different 
properties with varying crystal alignments, it is undesirable in the shape memory effect and the use of copper is once again justified.

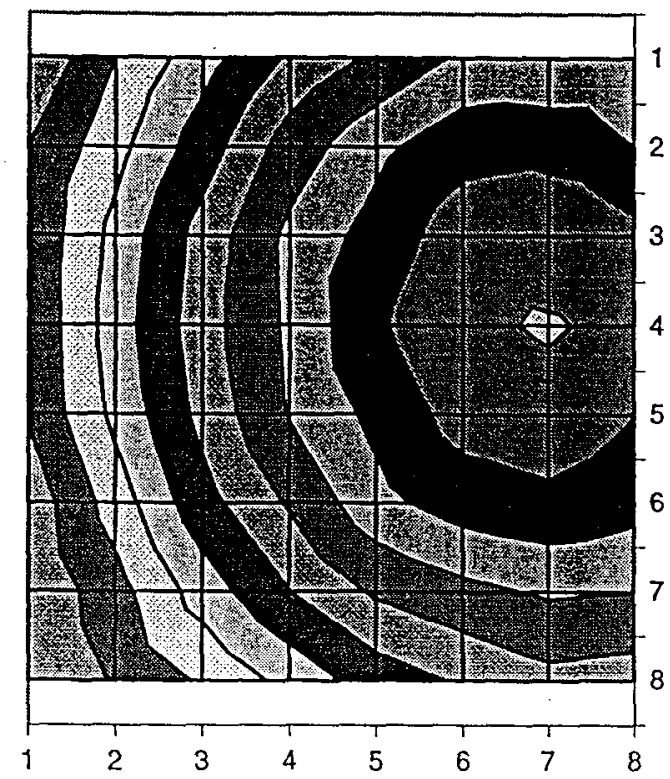

\begin{tabular}{|r|}
\hline $2.300-2.400$ \\
$\square 2.200-2.300$ \\
$\square 2.100-2.200$ \\
$\square 1.900-2.100$ \\
$\square 1.800-1.900$ \\
$\square 1.700-1.800$ \\
$\square 1.600-1.700$ \\
$\square 1.500-1.600$ \\
$1.400-1.500$ \\
$\square 1.300-1.400$ \\
\hline
\end{tabular}

Figure 3.2: Thickness map of one NiTiCu wafer showing that the greatest thickness of alloy was deposited directly in line with the sputtering target.

\subsubsection{SMA etch step}

\subsubsection{Photolithography pattern}

After sputter deposition of the $\mathrm{NiTiCu}$, wafers were hard-baked in an oven at $120^{\circ} \mathrm{C}$ for 20 min to remove any excess water, then soaked in HMDS vapor to assure good adhesion of photoresist. Negative AZ1518 photoresist [reference] was spun onto the frontside of wafers at $600 \mathrm{rpm}$ for $6 \mathrm{sec}$ and $2500 \mathrm{rpm}$ for $20 \mathrm{sec}$, yielding $-1.8 \mu \mathrm{m}$ of resist. The frontside resist was soft-baked on a hotplate at $100{ }^{\circ} \mathrm{C}$ for $60 \mathrm{sec}$. AZ1518 was then spun on the backside of wafers in the same manner as the frontside and soft-baked in an oven at $90^{\circ} \mathrm{C}$ for $20 \mathrm{~min}$. To create the ligament pattern seen in Figure 2.5, the mask seen in Figure 3.3 was used with ultraviolet (UV) contact photolithography. Exposed areas of resist where the clear field of the mask covered the wafer were removed with $\mathrm{AZ}$ developer and the wafers werc hard-baked at $120^{\circ} \mathrm{C}$ for $20 \mathrm{~min}$ to cure the remaining photoresist.

The mask in Figure 3.3 contains a pattern for 52 individual $1 \mathrm{~cm}$ square test chips. Forty of these are ligaments, ten are blank curvature test chips, and two are diaphragm test chips. During etching, the black areas of the mask were protected and the NiTiCu was unaffected, 
whereas the clear areas of the mask were etched away. The chips were numbered based on their $8 \times 8$ grid coordinates to identify them after they were cleaved apart.

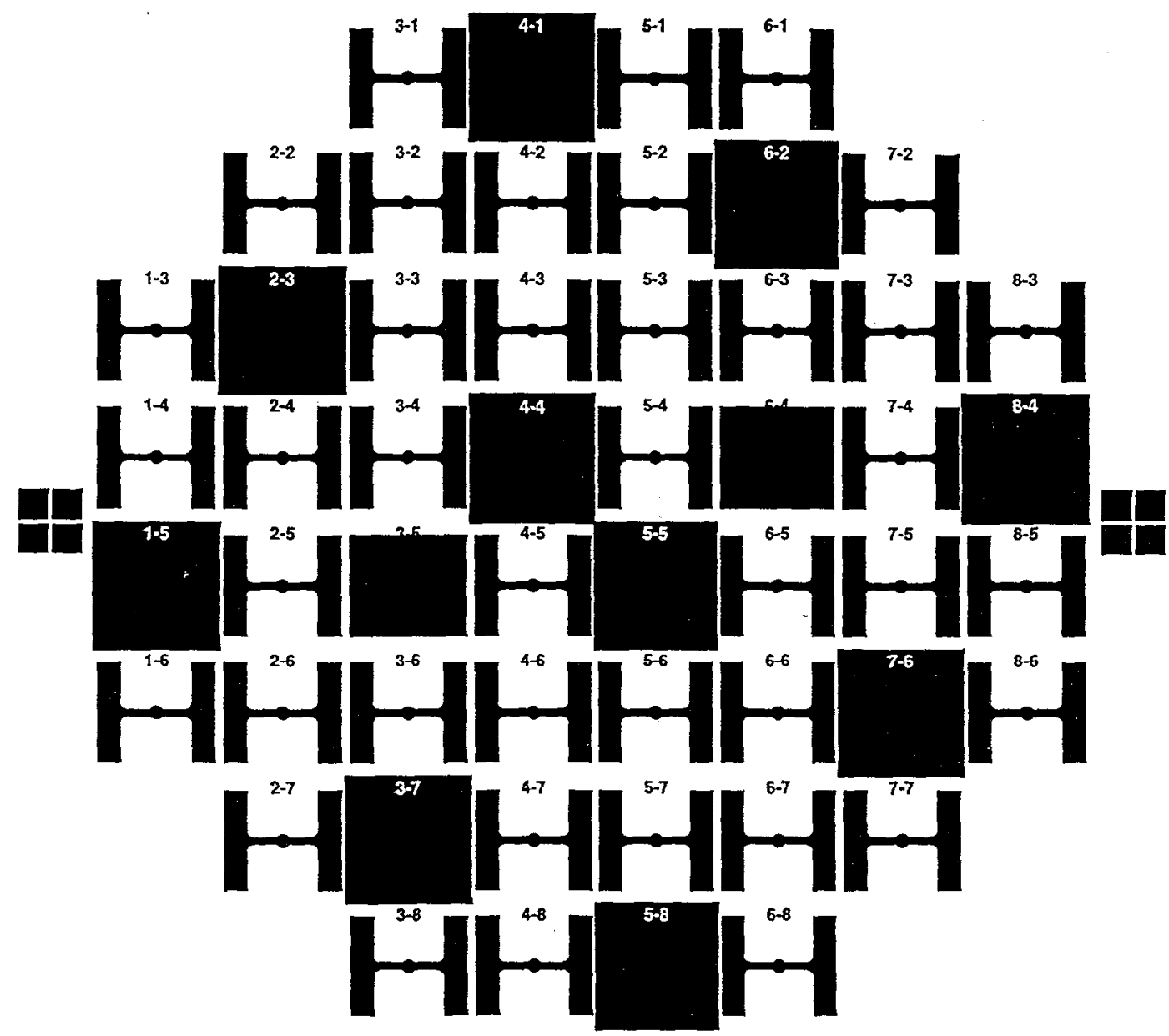

Figure 3.3: Contact photolithography mask used to pattern the NiTiCu into ligaments, diaphragms, and blank curvature test chips.

\subsubsection{2 $\mathrm{HCl}-\mathrm{HNO}_{3}-\mathrm{HF}$ wet chemical etch of $\mathrm{NiTiCu}$}

After photolithography, the wafers were ready to undergo etching of the film into the desired pattern. Upon soaking in a mixture of $20 \mathrm{HCl}: 20 \mathrm{HNO}_{3}: 1 \mathrm{HF}$, the $\sim 2 \mu \mathrm{m}$ of NiTiCu film that were not covered by photoresist were removed in approximately 5 min with no appreciable undercutting beneath the resist-covered areas. This mixture of hydrochloric acid, nitric acid, and hydrofluoric acid was chosen based upon their empirical reactions with the three metals in the alloy. The 20:20:1 ratio was determined after many attempts to find the right recipe of acids that would etch the film in a reasonable amount of time without etching the photoresist or the $\mathrm{SiO}_{2}$. The photoresist held up well to the acids used in the metal etch, but the problem arose of adding too much HF. Hydrofluoric acid was needed in the mixture to break the metal oxide so that the 
other acids could etch the three constituent metals in the alloy. Too much HF, however, would etch the $\mathrm{SiO}_{2}$ after breaking through the thin metal layer. If the $\mathrm{SiO}_{2}$ was etched away from any part of the wafer before all metal was etched, the nitric acid would begin to attack the silicon at a rate of a few tenths of a micron per minute.

After patterning of the ligaments, the thickness of the NiTiCu forming each ligament and blank chip was measured with a stylus tip profilometer. The measured thickness of each blank chip was used as the value $t_{f}$ for curvature tests described in Section 2.2 and by Equation 2-1. The thickness of each ligament was used for stress analysis of the ligaments as the value $h$ in Equations 2-19 and 2-20.

\subsubsection{Creation of free-standing ligaments}

\subsubsection{Patterning the backside of the wafer}

To create windows behind the ligaments and diaphragms and cleave lines to break up the test chips, the wafers underwent backside photolithography to create the pattern seen in Figure 3.4. In preparation for photolithography, the wafers were hard-baked at $120^{\circ} \mathrm{C}$ for 20 minutes and soaked in HMDS vapor for 5 minutes. Approximately $15 \mu \mathrm{m}$ of AZ4620 negative photoresist [reference] was spun onto the backside at $1750 \mathrm{rpm}$ for $9 \mathrm{sec}$ and then $1000 \mathrm{rpm}$ for $60 \mathrm{sec}$. Wafers were soft-baked in a $90^{\circ} \mathrm{C}$ oven for 9 minutes and aligned to the mask in Figure 3.4 using back side alignment and the alignment targets seen on the sides of the masks in Figure 3.3 and 3.4. After contact between the wafer and the mask was established, $8.07 \mathrm{~mW} / \mathrm{cm}^{2} \mathrm{UV}$ light exposed the resist for $83 \mathrm{sec}$ and the exposed resist was removed in $\mathrm{AZ}$ developer.

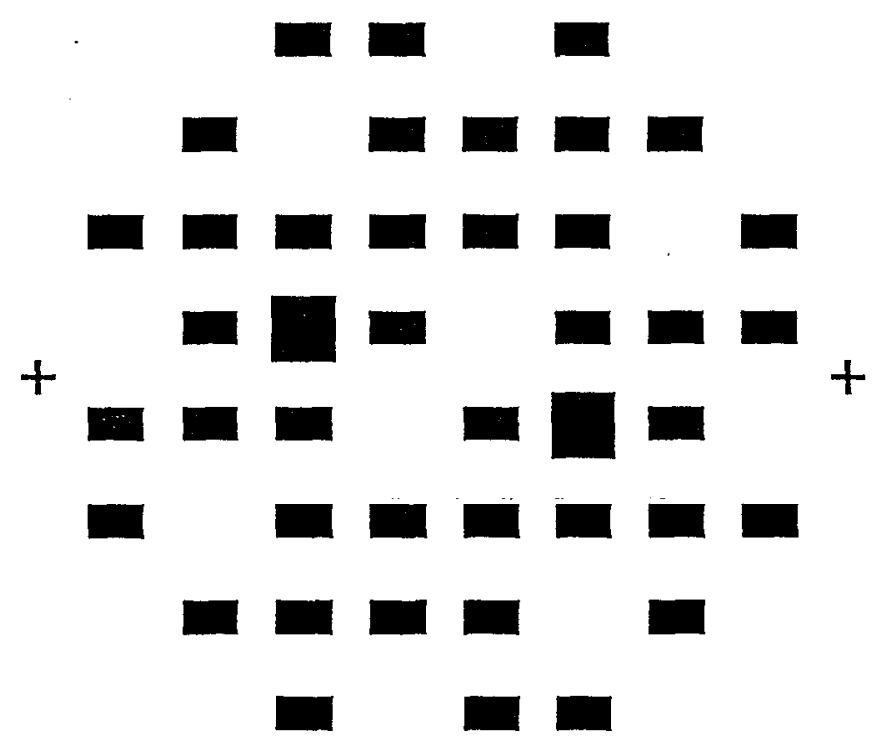

Figure 3.4: Backside alignment contact photolithography mask used for creation of windows behind ligaments and diaphragms. 
For bonding to a carrier wafer, AZ1518 was spun on the frontside at $600 \mathrm{rpm}$ for $6 \mathrm{sec}$ and $1000 \mathrm{rpm}$ for $20 \mathrm{sec}$, leaving a $\sim 4-5 \mu \mathrm{m}$ thick photoresist layer and filling in the void comers where patterned SMA met the wafer. The wafer was then soft-baked at $90^{\circ} \mathrm{C}$ for 15 minutes to partially cure the AZ1518. The 4" Si carrier wafer was prepared by spinning AZ1518 on the frontside at $600 \mathrm{rpm}$ for $6 \mathrm{sec}$ and $2500 \mathrm{rpm}$ for $20 \mathrm{sec}$. While this photoresist was still viscous and not hardened, the frontside of the carrier wafer was placed in contact with the frontside of the patterned wafer and held together under $40 \mathrm{~N}$ force for one hour. This sandwiched structure was then hard-baked at $120^{\circ} \mathrm{C}$ for 20 minutes to cure all the resist.

\subsubsection{Wet etch of $\mathrm{SiO}_{2}$}

In order to etch the silicon away beneath the ligaments, the uncovered $\mathrm{SiO}_{2}$ on the backside needed to be removed using a hydrofluoric acid solution buffered with acetic acid. The buffered HF solution etched the $0.6 \mu \mathrm{m}$ oxide layer without harming the silicon and with negligible etching of the patterned photoresist layer. After $\sim 5$ minutes of etching, the presence of exposed silicon was seen by its hydrophobic behavior as the buffered HF was repelled from the surface of the silicon.

\subsubsection{STS Advanced Silicon Etch}

The exposed silicon was etched from the backside of the patterned wafer in a Surface Technology Systems (STS) Advanced Silicon Etch (ASE) system [reference]. The plasma chemistry in this silicon etch followed a two step process of passivation and etching. For each etch cycle a passivation layer was laid down with the $\mathrm{C}_{4} \mathrm{~F}_{8}$ for $8 \mathrm{sec}$ and etching with $\mathrm{SF}_{6}$ removed the passivation material and some silicon for $12 \mathrm{sec}$. Plasma was created by a radiofrequency coil powered by $600 \mathrm{~W}$ and ions were directed with a platen powered by $130 \mathrm{~W}$. The etch took place under a 25 mTorr vacuum with active helium cooling on the backside of the carrier wafer. The $380 \mu \mathrm{m}$ of silicon was etched in two hours, exposing $\mathrm{SiO}_{2}$ windows from the frontside of the wafer with the ligaments and diaphragms visible through the oxide.

The carrier wafer was necessary for this etch because of the active helium cooling on the backside of the sandwich structure. Without cooling, the wafer would heat up quickly in the plasma environment and etch-rates and selectivity would decrease dramatically. Since the backside of the sandwich structure was pressurized with helium and the frontside was under vacuum, any holes made through a wafer would allow pressurization of the vacuum chamber and stop the etch through interlocks. Through attachment to a carrier wafer, holes were not allowed to go all the way through the sandwich and pressures were held on both sides of the wafers. 


\subsubsection{Technics RIE to etch $\mathrm{SiO}_{2}$ window}

With only the $\mathrm{SiO}_{2}$ windows remaining before the ligaments were free, the final etch step was completed. A Technics reactive ion etch (RIE) system [reference] was used to etch the remaining $\mathrm{SiO}_{2}$ from the wafers. Under a vacuum of $25 \mathrm{mTorr}$, a $150 \mathrm{~W}$ plasma was created with $20 \mathrm{sccm}$ of $\mathrm{CF}_{4}$ and $2 \mathrm{sccm}$ of $\mathrm{O}_{2}$, which etched the $0.6 \mu \mathrm{m}$ of $\mathrm{SiO}_{2}$ in 25 minutes.

After all $\mathrm{SiO}_{2}$ was removed from the wafer, the sandwich structure was soaked in acetone for two hours to dissolve the resist holding the wafers together and the wafer with freed ligaments was washed in acetone and isopropyl alcohol to remove all remnants of resist. The finished wafer seen in Figure 3.5 shows that $100 \%$ yield for a wafer was achievable due to the robustness of the NiTiCu ligaments in their martensite phase. The wafer was cleaved into the 52 test chips along the cleave lines made by the STS etch and testing was begun.

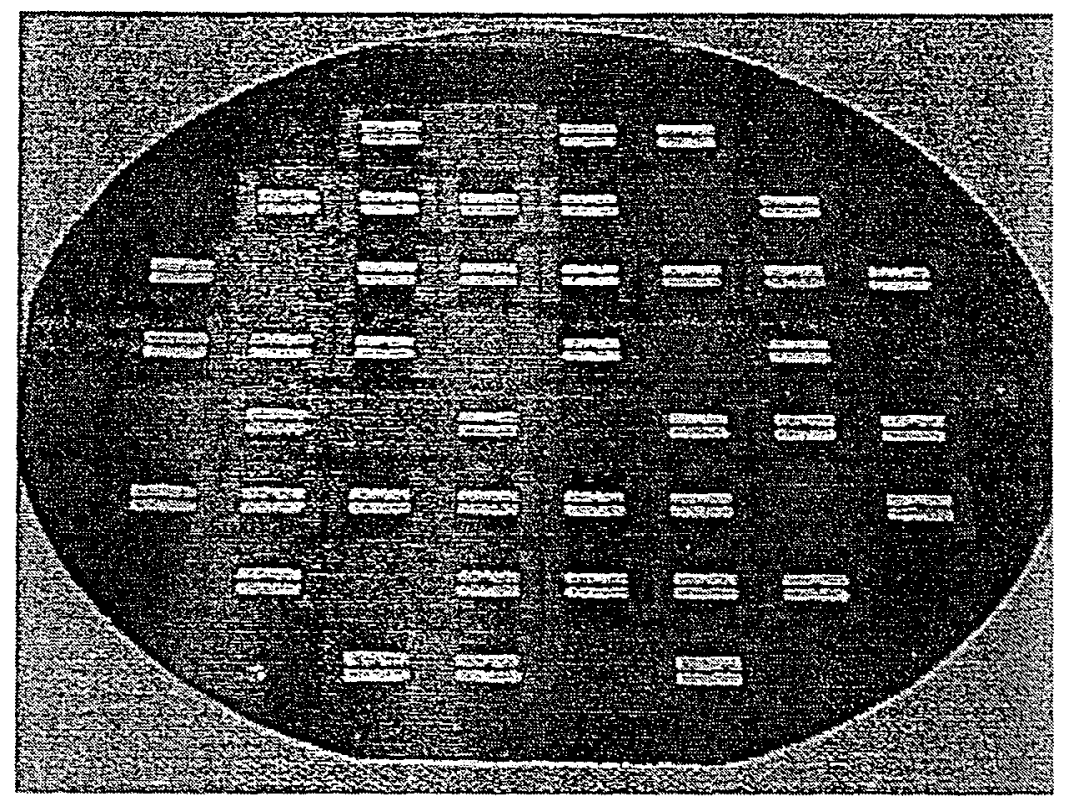

Figure 3.5: Photograph of a finished waver with 40 NiTiCu ligaments, 10 blanks, and 2 diaphragms, proving that $100 \%$ yield was achievable.

\subsection{Unsuccessful wafer processing attempts}

\subsubsection{Wet etching the Si wafer}

\subsubsection{Galvanic cell formation}

The first attempt to etch the silicon windows from beneath the ligaments was made using $44 \%$ wt. $\mathrm{KOH}$ at $90^{\circ} \mathrm{C}$ as a wet etchant without masking the $\mathrm{NiTiCu}$ on the frontside of the wafer. Etching all of the silicon with this method would take approximately two hours. This method failed because the NiTiCu was etched away by the $\mathrm{KOH}$ due to the formation of a galvanic cell 
between the alloy and the silicon. Nickel and titanium have electronegativities of $-0.257 \mathrm{~V}$ and

$-1.630 \mathrm{~V}$, respectively, and potassium has an electronegativity of $-2.931 \mathrm{~V}$ [reference]. A galvanic cell forms in a highly polarized medium (like $\mathrm{KOH}$ ) between the metal and the solution, with the metal anode giving up electrons and the solution cathode gaining electrons. The NiTiCu anode was oxidized by the galvanic reaction and etched away from the wafer. Metals tend to experience higher galvanic etch rates with higher difference in electronegativity between the cathode and the anode, so the potential caused between the nickel and the potassium solution was enough to rapidly etch away the metal [reference].

\subsubsection{CrAu masking unsuccessful}

In an attempt to mask the $\mathrm{NiTiCu}$ from the $\mathrm{KOH}$ solution to use the wet silicon etchant, a chrome-gold mask was sputtered onto the wafer with thickness of $0.05 \mu \mathrm{m}$ of chrome as an adhesion layer to the $0.5 \mu \mathrm{m}$ layer of gold. This was unsuccessful because the electronegativities of chrome and gold are -0.744 and $1.498 \mathrm{~V}$, respectively, and a high potential galvanic cell formed between the $\mathrm{CrAu}$ and the NiTiCu wherever any of the NiTiCu was exposed due to the large difference in electronegativity between the gold and the potassium and between the gold and the titanium. Since the mask did not successfully reach down into corners where the NiTiCu met the silicon, $\mathrm{KOH}$ got in to form the galvanic cell. This caused the CrAu mask to bubble away from the Si and $\mathrm{NiTiCu}$, exposing more NiTiCu to $\mathrm{KOH}$ and eventually causing the entire NiTiCu film to etch.

\subsubsection{Pre-etching frontside cleave lines}

In order to cleave the wafers into $1 \mathrm{~cm}$ square chips after micromachining the ligaments, diaphragms, and blank chips, an attempt to pre-etch cleave lines in the frontside of the wafers before NiTiCu deposition was made. One hundred $\mu \mathrm{m}$ deep channels were etched in the frontside in the $1 \mathrm{~cm}$ square grid pattern. This did not work, however, because when the wafer was bonded to the carrier wafer with photoresist, air bubbles remained in the channels. When the sandwich structure was placed in the STS etcher and pumped down to 25 mTorr, the vacuum caused the air bubbles between the wafers to force the wafers apart. With the bond between the wafers broken, the STS etch could not be completed and windows could not be made in the wafer.

\subsubsection{Wet etch of NiTiCu without $\mathrm{SiO}_{2}$ layer}

As mentioned in Section 3.2.1, the $\mathrm{SiO}_{2}$ layer is necessary to protect the $\mathrm{Si}$ wafer during the SMA etch. Because the nitric acid $\left(\mathrm{HNO}_{3}\right)$ in the NiTiCu etchant reacts with the Si to form 
$\mathrm{SiO}_{2}$ and the hydrofluoric acid $(\mathrm{HF})$, reeded to remove the oxide from the $\mathrm{NiTiCu}$, etches the newly formed $\mathrm{SiO}_{2}$, the combination of these acids etches $\mathrm{Si}$ rather quickly. Since the acid mixture etches through the metal on the outer edges of the wafer more quickly than the center due to the difference in thickness, the Si on the outside undergoes etching while the metal in the center is still not removed. To ensure no $\mathrm{Si}$ etching, the $\mathrm{SiO}_{2}$ layer is depositcd. There is no worry of the oxide layer etching by the small part of HF in the mixture because it is $0.6 \mu \mathrm{m}$ thick and only $\sim 0.02 \mu \mathrm{m}$ was removed while the $2 \mu \mathrm{m}$ of NiTiCu etched, leading to a selectivity of about 100:1.

\subsubsection{Wet etch of NiTiCu using $100^{\circ} \mathrm{C} \mathrm{H}_{3} \mathrm{PO}_{4}$}

In an effort to not deposit $\mathrm{SiO}_{2}$ on the wafer, alternative acids were considered to etch the $\mathrm{NiTiCu}$ in the desired pattern. Concentrated phosphoric acid $\left(\mathrm{H}_{3} \mathrm{PO}_{4}\right)$ was tested at room temperature and at $100^{\circ} \mathrm{C}$ as an etching acid because it has been empirically proven to etch $\mathrm{Ti}$ and $\mathrm{Cu}$ at room temperature and $\mathrm{Ni}$ at elevated temperature. This attempt proved to be complctely unsuccessful at room temperature and did not etch the entire film at $100^{\circ} \mathrm{C}$. A black precipitate was formed during the etch at elevated temperature and the etch stopped before the entire film was gone. For these reasons, $\mathrm{H}_{3} \mathrm{PO}_{4}$ was not used as the etching acid for the NiTiCu films.

\subsubsection{STS etch without $\mathrm{SiO}_{2}$ layer}

During the experiments without $\mathrm{SiO}_{2}$ coatings on the wafers, another reason to use $\mathrm{SiO}_{2}$ was discovered. After the NiTiCu ligaments were etched using $\mathrm{H}_{3} \mathrm{PO}_{4}$, the STS etch to free the ligaments was begun. By the time the etch was almost complete, the backside of the ligaments began to char and the etch was stopped. Upon examination under a microscope, it was observed that silicon remained on the back of the ligaments where they were not charred. This small amount of silicon caused the ligaments to be very brittle and unusable for testing. 


\section{Chapter 4: Results and discussion}

\subsection{Calibration data}

\subsubsection{DVRT calibration}

The first step in understanding the output of the DVRT was to calibrate displacement or compression of the spring to the output voltage. To perform this calibration, the DVRT head was lowered with the precision micrometer against a solid aluminum block until contact was made, at which point the output was found at zero displacement as $-2.145 \mathrm{~V}$. The spring was then compressed by $10 \mu \mathrm{m}$ increments, measured by the precision micrometer, and the output voltages were recorded. This calibration data is seen in Figure 4.1, showing a slight nonlinear regime between 1000 and $1500 \mu \mathrm{m}$ of spring compression, where the slope changes from $1.24 \mathrm{mV} / \mu \mathrm{m}$ below $1000 \mu \mathrm{m}$ compression to $1.51 \mathrm{mV} / \mu \mathrm{m}$ above $1500 \mu \mathrm{m}$ compression. Calibration was completed three times with negligible difference in DVRT output to prove the repeatability of DVRT measurements.

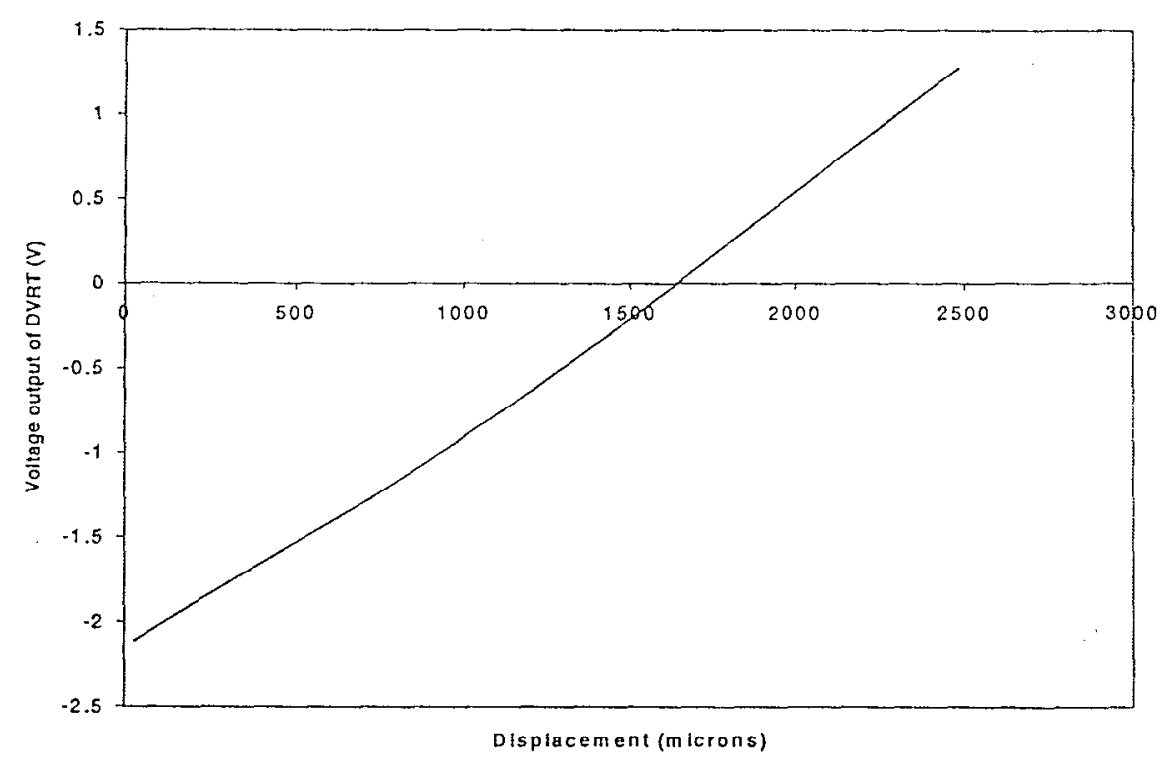

Figure 4.1: Calibration data for the DVRT indicated a slight nonlinearity between 1000 and $1500 \mu \mathrm{m}$ of spring compression.

Because of the slight nonlinearity, the calibration constants (in $\mathrm{mV} / \mu \mathrm{m}$ ) were not used to determine the displacements at given voltages. Instead, a chart of calibration data was created and DVRT output voltages were converted to displacements by way of spreadsheet calculations. A partial example of this calibration chart is seen in Table 4.1, showing that individual DVRT 
outputs could be indexed to determine displacements. Because the DVRT voltage would float over time, the initial, uncompressed output of the DVRT was subtracted from the original zero point $(-2.145 \mathrm{~V})$ and the difference was added to subsequent DVRT measurements to compensate for floating outputs and normalize all measurements.

Table 4.1: DVRT Calibration Chart

\begin{tabular}{|c|c|c|c|c|c|c|c|}
\hline $\begin{array}{c}\text { Voltage } \\
(V)\end{array}$ & $\begin{array}{c}\text { Displacement } \\
(\mu \mathrm{m})\end{array}$ & $\begin{array}{c}\text { Voltage } \\
(\mathrm{V})\end{array}$ & $\begin{array}{c}\text { Displacement } \\
(\mu \mathrm{m})\end{array}$ & $\begin{array}{c}\text { Voltage } \\
(\mathrm{V})\end{array}$ & $\begin{array}{c}\text { Displacement } \\
(\mu \mathrm{m})\end{array}$ & $\begin{array}{c}\text { Voltage } \\
(\mathrm{V})\end{array}$ & $\begin{array}{c}\text { Displacement } \\
(\mu \mathrm{m})\end{array}$ \\
\hline-2.145 & 0 & -2.129 & 13 & -2.113 & 26 & -2.096 & 39 \\
\hline-2.144 & 1 & -2.128 & 14 & -2.111 & 27 & -2.095 & 40 \\
\hline-2.143 & 2 & -2.126 & 15 & -2.110 & 28 & -2.094 & 41 \\
\hline-2.142 & 3 & -2.125 & 16 & -2.109 & 29 & -2.093 & 42 \\
\hline-2.140 & 4 & -2.124 & 17 & -2.108 & 30 & -2.091 & 43 \\
\hline-2.139 & 5 & -2.123 & 18 & -2.106 & 31 & -2.090 & 44 \\
\hline-2.138 & 6 & -2.121 & 19 & -2.105 & 32 & -2.089 & 45 \\
\hline-2.136 & 7 & -2.120 & 20 & -2.104 & 33 & -2.088 & 46 \\
\hline-2.135 & 8 & -2.119 & 21 & -2.103 & 34 & -2.086 & 47 \\
\hline-2.134 & 9 & -2.118 & 22 & -2.101 & 35 & -2.085 & 48 \\
\hline-2.133 & 10 & -2.116 & 23 & -2.100 & 36 & -2.084 & 49 \\
\hline-2.131 & 11 & -2.115 & 24 & -2.099 & 37 & -2.083 & 50 \\
\hline-2.130 & 12 & -2.114 & 25 & -2.098 & 38 & -2.081 & 51 \\
\hline
\end{tabular}

\subsubsection{DVRT spring constant}

To convert spring compression measurements to reaction forces, the DVRT spring constant was calibrated. In order to make this calibration, a 1/4" thick aluminum plate was drilled and threaded and the DVRT was screwed into it with the head at the top. A top hat interface fixture was machined from aluminum to fit over the DVRT head and allow the stacking of washers on the fixture, making loading of the DVRT spring possible. The top hat was weighed and placed on the head as an initial load, and washers were weighed and stacked to continue loading. By multiplying the mass of washers and the top hat by the gravitational constant $(9.81$ $\mathrm{m} / \mathrm{s}^{2}$ ), the reaction force of the compressed spring was determined for each loading point. When the output voltage of the DVRT was measured at each of these loading points, the displacements were found by the indexing method discussed in the previous section and the curve in Figure 4.2 was created and the DVRT spring constant was detcrmincd. Two individual calibrations were performed, yielding spring constants of $0.184 \mathrm{~N} / \mathrm{mm}(+)$ and $0.188 \mathrm{~N} / \mathrm{mm}(0)$ as seen in the plotted data. The spring constant used for force calculations was therefore $0.186 \mathrm{~N} / \mathrm{mm}$, with uncertainty of $\pm 0.002 \mathrm{~N} / \mathrm{mm}$. 


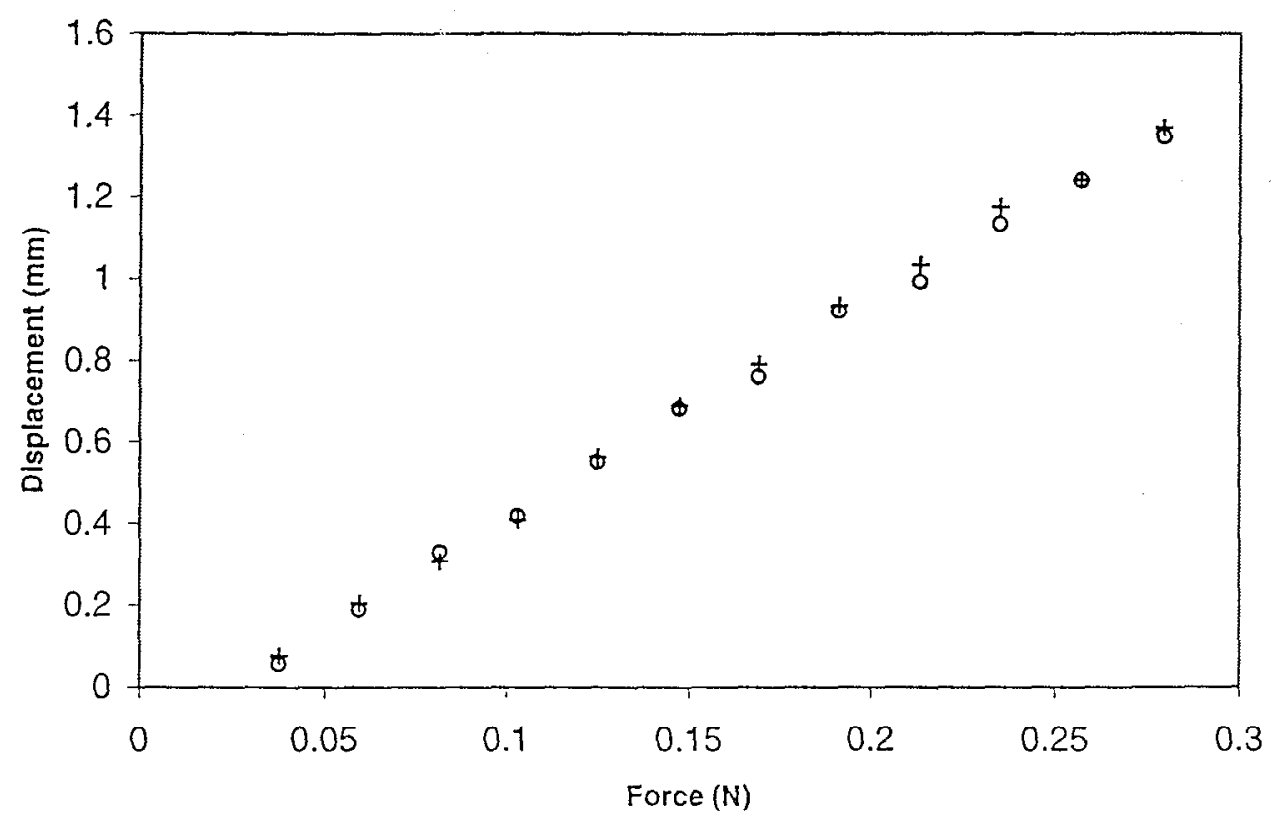

Figure 4.2: Force versus displacement calibration of DVRT spring.

\subsection{Curvature tests}

\subsubsection{Transformation temperatures}

Curvature tests were performed to quickly confirm shape memory behavior, measure residual stresses, and find transition temperatures of the film. A plot of stress vs. temperature for nine chips from the same substrate is shown in Figure 4.3. Transformation temperatures were $A_{s}=50^{\circ} \mathrm{C}, A_{f}=61^{\circ} \mathrm{C}, M_{s}=58^{\circ} \mathrm{C}$ and $M_{f}=37^{\circ} \mathrm{C}(A=$ austenite, $M=$ martensite, $s=$ start, $f=$ finish $)$.

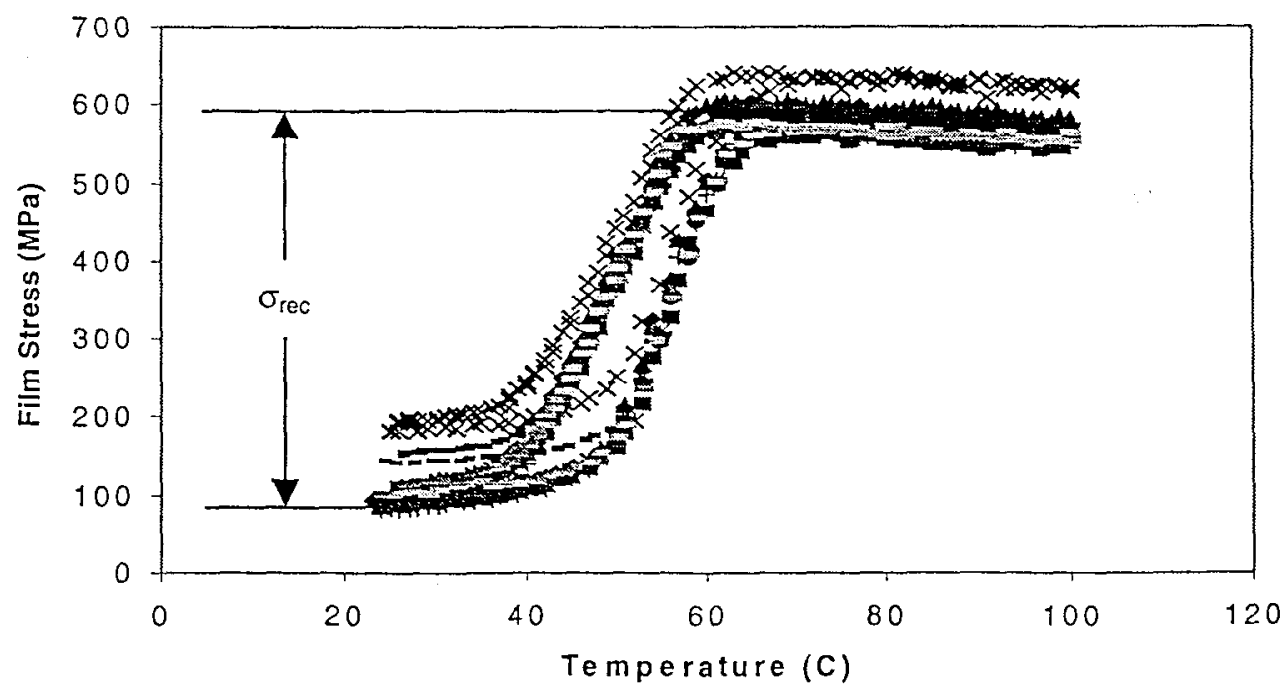

Figure 4.3: Curvature measurements of nine chips indicate the uniformity of shape memory effect across a wafer. 
When comparing the data in Figure 4.3 to the predicted film stress versus temperature in Figure 2.1, it is readily noticed that the shape of the experimental curve was accurate to theory. The hysteresis width is $3{ }^{\circ} \mathrm{C}$ at the top of the curve $\left(A_{r}-M_{s}\right)$ and $13^{\circ} \mathrm{C}$ at the bottom of the curve $\left(A_{s}-M_{f}\right)$. The curve slopes downward in the austenite phase as expected due to the different coefficients of thermal expansion. The two chips showing discrepancy in this plot were located on the outer edges of the wafer, and had thinner films on them with more uncertainty in the film thickness measurements, which could account for the differences. The uncertainty in thickness measurements could also account for the $50 \mathrm{MPa}$ range of maximum film stress in the main grouping of measurements, centered at $570 \mathrm{MPa}$, yielding an $8.8 \%$ error range. Since the film stress is inversely proportional to the film thickness as governed by the Stoney Equation (Eq 2-1), only $4.4 \%$ of inaccuracy in the thickness measurements would allow the range of error seen in Figure 4.3.

\subsubsection{Recoverable stresses}

The recoverable stress in the film is seen as the difference between the maximum film stress and the martensite yield stress, or residual martensite stress, in the film. This stress is a special case for the curvature measurement and is dependent upon the boundary conditions of the interface between the film and the substrate. Because it is a special case, the recoverable stress in the film differs between curvature measurements and ligament tests, as we will see later in Section 4.3.4. The recoverable stress in this case, however, averaged across the nine test chips used to plot Figure 4.3 , was $485 \mathrm{MPa}$. The individual recoverable stresses for the chips were 512 , $482,519,460,485,492,494,449$, and $476 \mathrm{MPa}$.

\subsubsection{Residual stress and strain}

The individual residual stresses found from the initial curvature measurements of the nine blank test chips were $92,90,89,184,99,92,80,142$, and $101 \mathrm{MPa}$. The average across all chips was $108 \mathrm{MPa}$, but if the two abnormal chips with 184 and $142 \mathrm{MPa}$ residual stress were excluded, the average martensite residual stress across the wafer was $92 \mathrm{MPa}$. The residual stress in the austenite phase, seen as the maximum film stress in Figure 4.3, was averaged across all nine chips as $592 \mathrm{MPa}$ and across the seven grouped chips as $585 \mathrm{MPa}$. Using Eq. 2-18 to find the uniaxial residual stress for a freed film indicated that ligaments were tensioned by $72 \mathrm{MPa}$ of residual stress if the larger average was used and $62 \mathrm{MPa}$ if the two abnormal chips were not included in the calculation.

The residual strain was calculated from the gap width $e$ discussed in Section 2.5.2.1 and Eq. 2-12. The gap width was measured with a reticle lens under a microscope as $10 \mu \mathrm{m}$ for two 
test ligaments that were fractured during cleaving of ligaments and blank chips from the wafer. Half of the gap width was subtracted from the designed length of one ligament leg $(2 \mathrm{~mm})$ to give the initial unstrained ligament length. When the designed length was divided by this difference and the natural logarithm was taken of the quotient, the residual uniaxial true strain was found to be $0.00501 \varepsilon$.

By finding the ratio of stress to true strain, an initial Young's modulus calculation of the material was found. These residual stress and strain therefore yielded a martensite Young's modulus of $14.4 \mathrm{GPa}$ if all nine curvature chips were used in the calculation and $12.4 \mathrm{GPa}$ if the two abnormal chips were discounted.

\subsection{Static and actuation tests}

\subsubsection{Stress-strain curves}

Static loading tests were performed on five heated ligaments to find austenite characteristics and five room temperature ligaments to determine the martensitic reaction to loading. The results from these tests are displayed in Figure 4.4. These results are offset by the residual strain discussed in the previous section, but not the residual stress because force measurements by the DVRT spring directly yiclded the absolute tensile stress in the films.

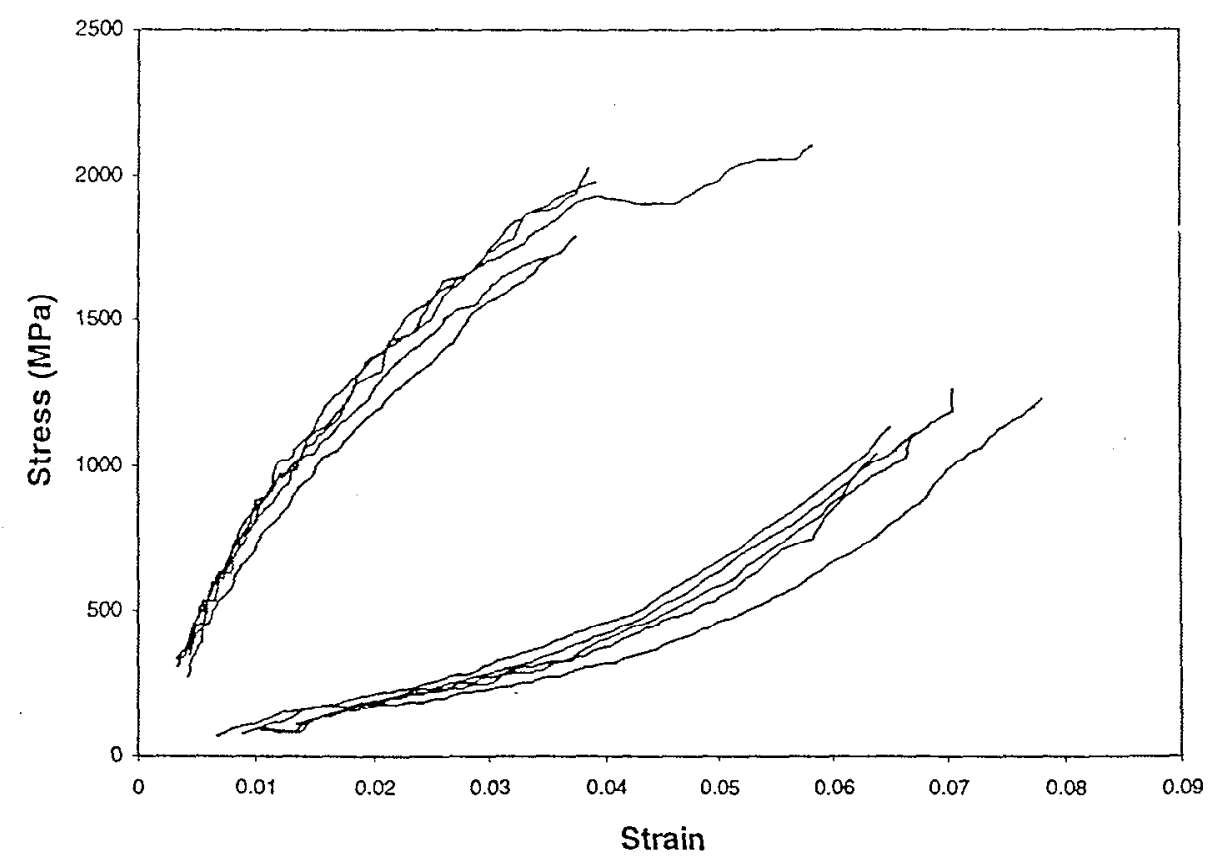

Figure 4.4: Stress versus strain for five austenite ligaments (higher slope) and five martensite ligaments (lower slope), offset by initial strain. Initial stress was not used as an offset because stress measurements were absolute. 
As a basis of comparison, stress-strain plots were created for ligaments undergoing the actuation tests described in Section 2.4.1. When these were plotted against the static test results (Figure 4.5) large differences in behavior were observed between testing methods. The bold lines in the figure represent the static test data seen in Figure 4.4, while the lighter lines show the behavior of ligaments during actuation testing. The ligaments tested by the static method displayed slightly more stiffness in the martensite phase and much more stiffness in the austenite phase. This indicates that during actuation, less stress was recovered when passing from martensite to austenite than in the static tests where the ligaments never entered the martensite phase during testing. The material was more ductile in its austenite phase when it was transforming from martensite under stress than when it underwent no transformation, but stayed in one phase during loading. Full transformation from martensite to austenite was inhibited by higher stresses in the film, but simply adding stress to an austenitic ligament would not cause as much of a backwards transformation to martensite. Therefore, less martensitic material existed in the ligaments tested by the static method than those tested with actuation.

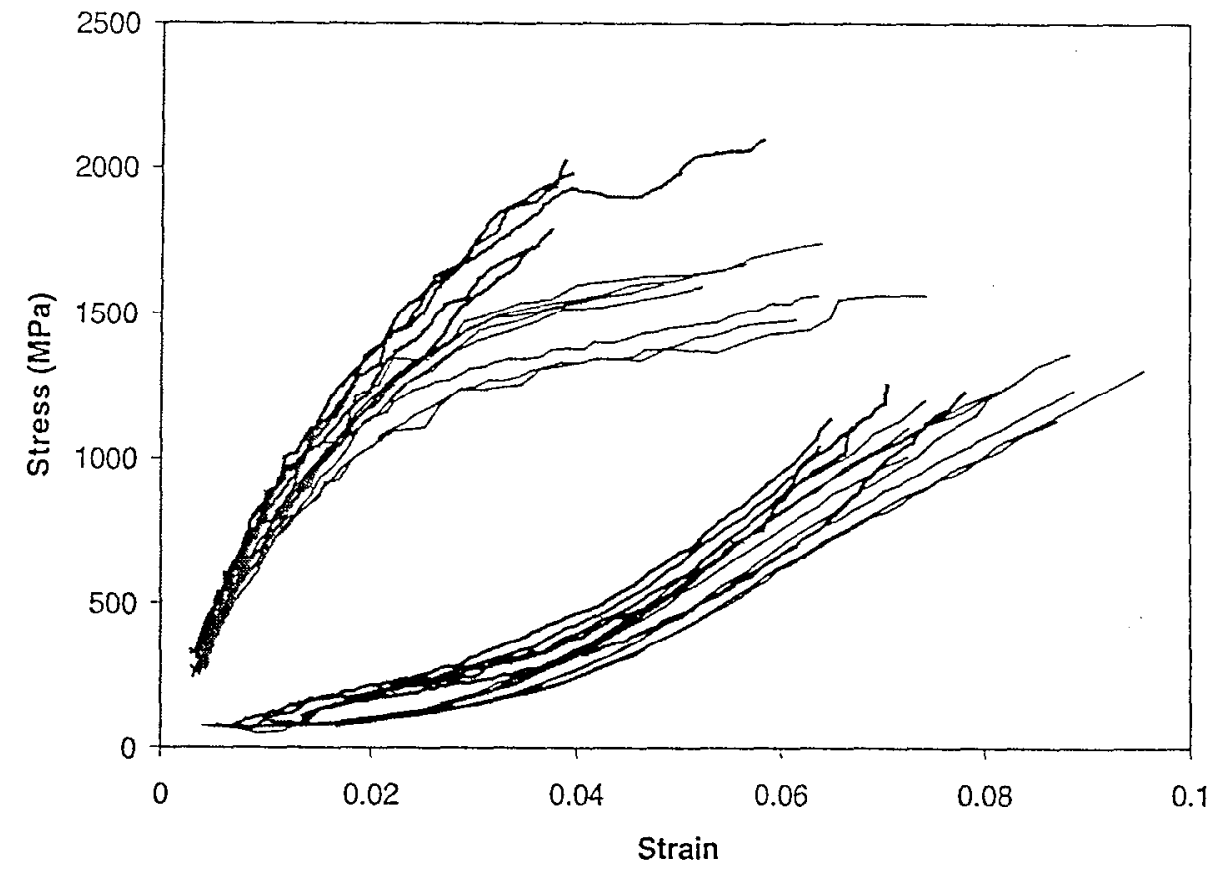

Figure 4.5: Stress versus strain for static austenite and martensite tests (bold lines) compared to actuation tests, going between martensite and austenite (lighter lines).

\subsubsection{Approximate modulus of elasticity}

In order to determine the plate modulus $E /\left(1-v^{2}\right)$ of each phase of the NiTiCu films, the slope of the stress-strain curves were measured for specified ranges and averaged over all samples 
on which static tests were performed. The appropriate linear ranges for martensite modulus were 1.0 to $3.0 \%$ strain and for austenite modulus were 0.5 to $1.5 \%$ strain. The martensite modulus was found as $8.5 \pm 3.3 \mathrm{GPa}$ and the austenite modulus was equal to $63 \pm 12 \mathrm{GPa}$. The error measurements in this case wcre cqual to twice the standard deviation of the values over which the measurement was averaged.

Unfortunately, the plate modulus approximations do not reveal very much about the actual experimental stress-strain curves because they are indicative of only small, linear portions. Rather than characterizing the curves with a normal linear equation where stress is proportional to strain, characteristic functions were determined that would better fit the data, as seen in Figure 4.7. For the austenite curve, a power function was found to match stress and strain with a correlation factor of 0.9724 :

$$
\sigma=22311 M P a \times \varepsilon^{0.7322}
$$

And for the martensite curve, a quadratic function was found with a correlation factor of 0.9584 . This function is inaccurate at low strains because it does not intersect the data at the origin, but captures the behavior of the martensite as twin motion subsides.

$$
\sigma=193453 \mathrm{MPa} \times \varepsilon^{2}+795.06 \mathrm{MPa} \times \varepsilon+76.538 \mathrm{MPa}
$$

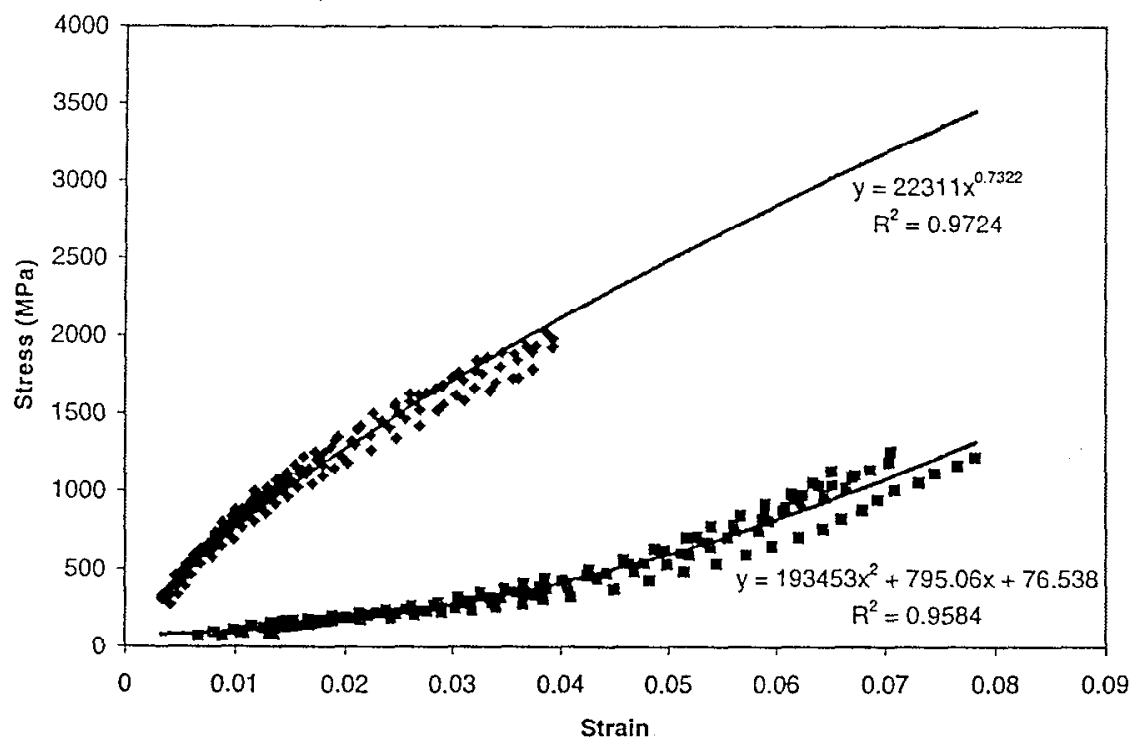

Figure 4.6: Approximate functions for the static test stress-strain curves were found as a power function for the austenite curve and a quadratic function for the martensite curve. Both functions had over $95 \%$ correlation constants. 


\subsubsection{Recoverable stress}

During ligament actuation, the material behavior would in effect move from a point on the martensite stress-strain curve to one on the austenite curve. As described in Section 2.6.2, the recoverable stress is the difference between the stress in the film in the two operating points. Stress would increase in the film as it further compressed the DVRT spring until full transformation had occurred. An example of the recoverable stress found in a ligament during actuation testing was plotted with respect to the initial true strain in Figure 4.7. The error bars seen in this and the plots that follow are explained in Appendix A.

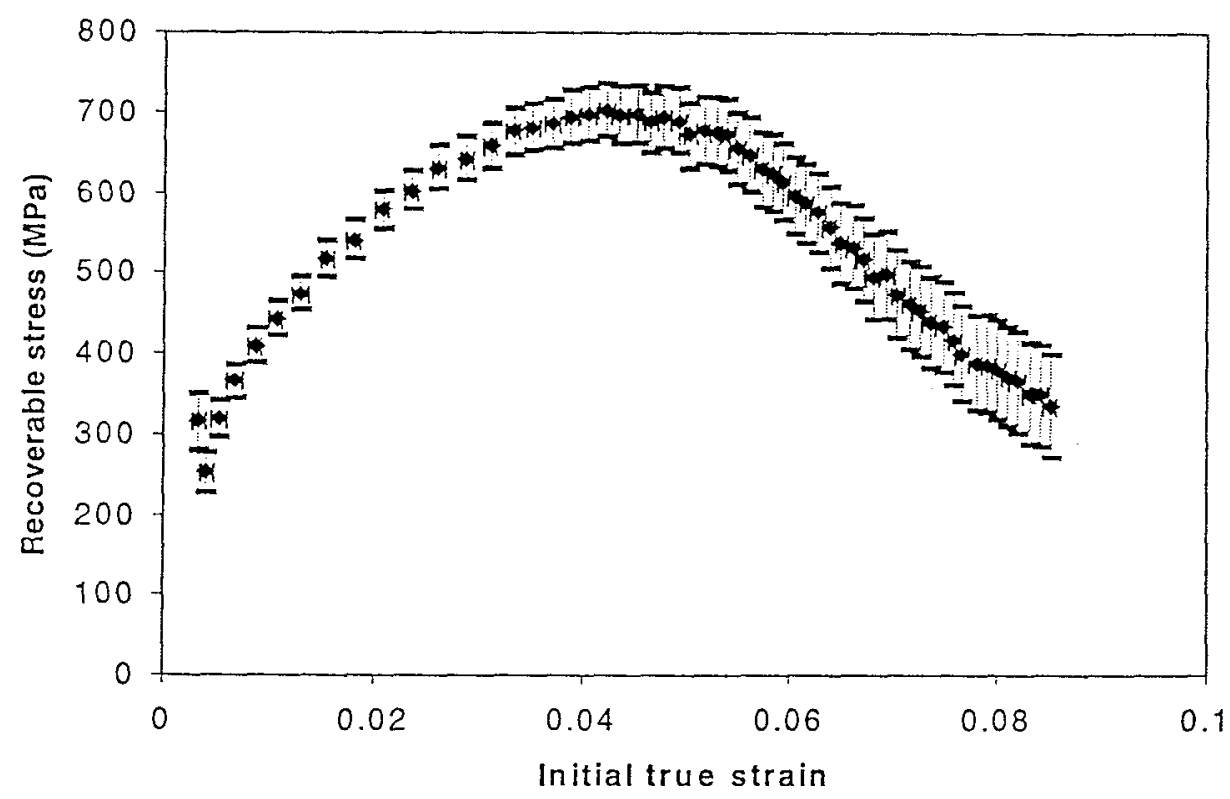

Figure 4.7: The recoverable stress data for one ligament plotted against the initial true strain in the ligament, including associated error.

The results for this ligament were typical of all ligaments, with the recoverable stress peaking at around $4.4 \%$ initial true strain and then falling off at a linear rate of roughly $125 \mathrm{MPa}$ per $1 \%$ true strain. Maximum recoverable stresses of $700 \mathrm{MPa}$ were higher than the normal recoverable stresses seen in curvature testing or found in the literature because loaded actuation of released films had never been done before. Without the boundary effects curvature specimens possess, freestanding ligaments have more freedom to actuate and therefore recover their stress. By comparison of Figure 4.7 and 4.5 , one can assume that even more recoverable stress is achievable by moving all the way from the martensite curve to the real austenite curve found in static measurements, rather than the curve obtained during these actuation measurements. 


\subsubsection{Recoverable strain}

The difference between the austenite true strain and the martensite true strain was found during actuation tests and is plotted with respect to the initial true strain in Figure 4.8. This data was for one ligament, but the shape of the curve was typical of the behavior of all ligaments, with a peak recoverable strain occurring at $5.8 \%$ initial true strain. The thickness of the ligament used to create these plots was $2.26 \mu \mathrm{m}$ and the maximum recoverable strain was $3.0 \%$. Maximum values for these tests differed with ligament thickness even though the recoverable stress was the same for the ligaments. Thinner ligaments would produce less actuation with the same recoverable stress because there was less total tensile force created during ligament actuation. In comparison, a $1.50 \mu \mathrm{m}$ thick liganent had maximum recoverable strain of $2.6 \%$, but experienced this peak at the same $5.8 \%$ initial true strain.

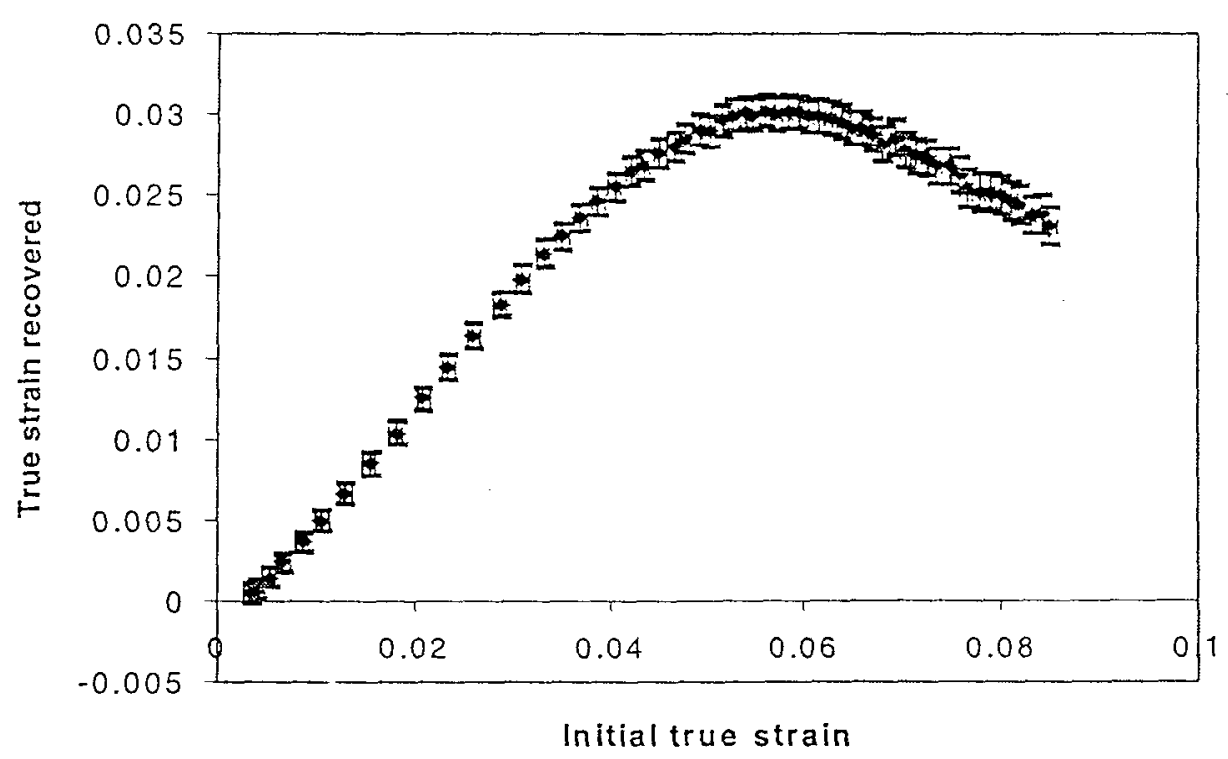

Figure 4.8: The recoverable strain for one ligament plotted against the initial true strain in the ligament, including associated error.

While this data is useful by showing how much strain is recovered over a range of initial strains, the percentage of strain recovered is more telling because it displays the portion of strain recovered under loading. Interpretation of the percentage data gives an idea of what percentage of twins are recovered in the phase transformation from martensite to austenite. The percentage of strain recovered under a load was plotted versus initial true strain for one ligament and is seen in Figure 4.9. The data indicates that the material recovers a higher proportion of its initial strain as more load is added up to $3.3 \%$ initial strain, at which point this percentage ratio begins to diminish. The maximum percentage of strain recovered was $64 \%$ at $3.3 \%$ initial true strain; 
therefore the recoverable stress at that data point was $0.64 \times 3.3 \%=2.1 \%$, seen in Figure 4.8 . The initial rapid rise in percentage of strain recovered is explained by the fact that a portion of the strain is not recoverable because it occurs before twin displacement. As soon as twins begin displacing relative to one another, the percentage of strain recovered rises rapidly to its plateau between $2.0 \%$ and $4.5 \%$ initial truc strain.

A slow, steady fall-off is seen after the plateau, where a lower volume fraction of twins are being recovered. To understand this lower rate of recovery we first look to the ClausiusClapeyron relationship for shape memory materials:

\section{Clausius-Clapeyron Equation (Eq 4-3)}

During actuation measurements, the power through the ligaments was maximized for small loads and deflections. The energy balance of the system requires that energy be input to transform martensite twins to $\mathrm{BCC}$ for the material to remember its shape. As loads are increased, more energy is bcing focused on twin deformation, requiring higher energies to fully transform the twins. Since actuation power did not vary within individual actuation tests, the higher power needed was not provided, and stress-induced twin formation occurred. When loads applied and then were released before heating, the material would regain its original shape with unnoticeable plastic deformation in the ligament, thus recovering all of its initial true strain. For that reason, it is stressed that the decreasing percentage of true strain recovered and volume fraction of twin recovery occurs solely because of higher stresses in the ligaments.

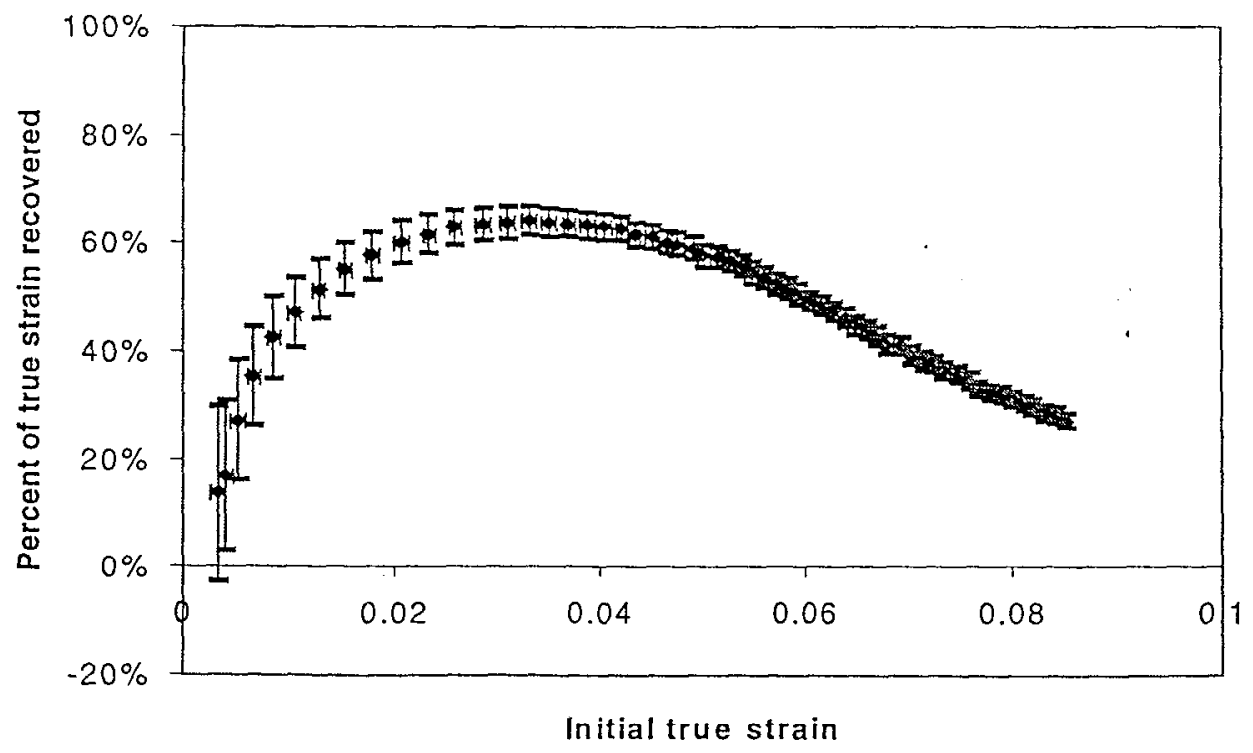

Figure 4.9: The percentage of true strain recovered for one ligament plotted against the initial true strain, including associated error. 


\subsubsection{Achievable forces and displacements}

The material's engineering properties, when coupled with the geometry of experimental systems, prove to be useful for the design of $\mu$-actuators employing SMA materials. The ligament example investigated in this paper is somewhat scaled up from typical $\mu$-devices with dimensions in millimeters rather than microns. However, since the films arc only $2 \mu \mathrm{m}$ thick, they can still give a good idea of what range of actuation deflections and forces are possible with freestanding SMA films.

The actuation forces and displacements created by the same ligament used to plot Figures 4.7-4.9 are seen in Figure 4.10. The data for force and displacement has the same graphical shape because the force measurement was simply the actuation deflection created during ligament heating multiplied by the spring constant of the DVRT spring. The deflection plotted here was that of the center of the ligament, thus the change in compression of the DVRT spring between martensite and austenite states. Maximum values of $56 \mathrm{mN}$ force and $300 \mu \mathrm{m}$ deflection were seen at $730 \mu \mathrm{m}$ initial micrometer displacement $\left(d_{m}\right)$. The basic shape of this curve was similar for all ligaments that underwent actuation testing, but maximum values differed with film thickness. Because thinner ligaments had the same recoverable stress with lower cross-sectional area, they were also under less tensile force and could thus produce less actuation force. Because less actuation force was produced, and the force was directly proportional to the deflection, less deflection was also apparent.

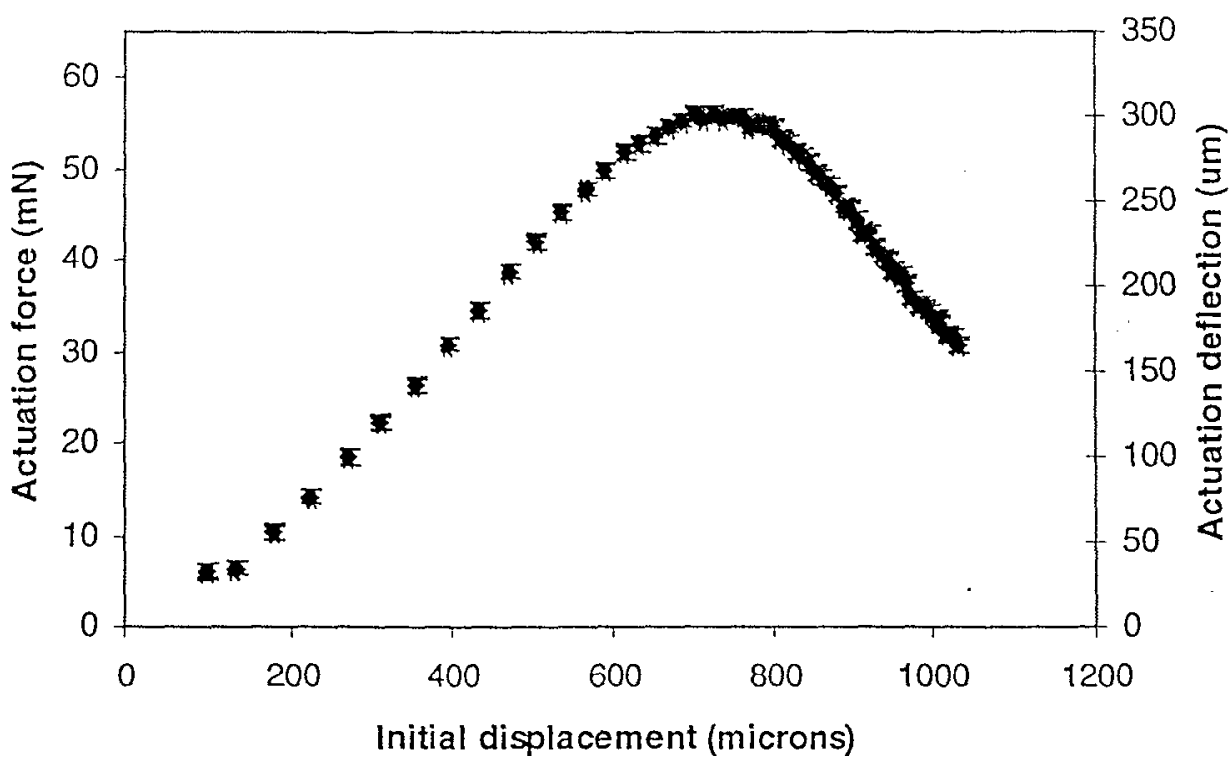

Figure 4.10: Actuation force and deflection of one ligament plotted with respect to initial micrometer displacement including associated error. 


\subsubsection{Ligament resistance}

In order to get an idea of the power requirements for ligament heating, the resistance of each ligament was initially measured with a digital multimeter. To verify the accuracy of these measurements, they were plotted with respect to film thickness (Figure 4.11) with the expcctation that an inverse proportionality would be observed in accordance with the theoretical resistance relationship in Equation 2-24.

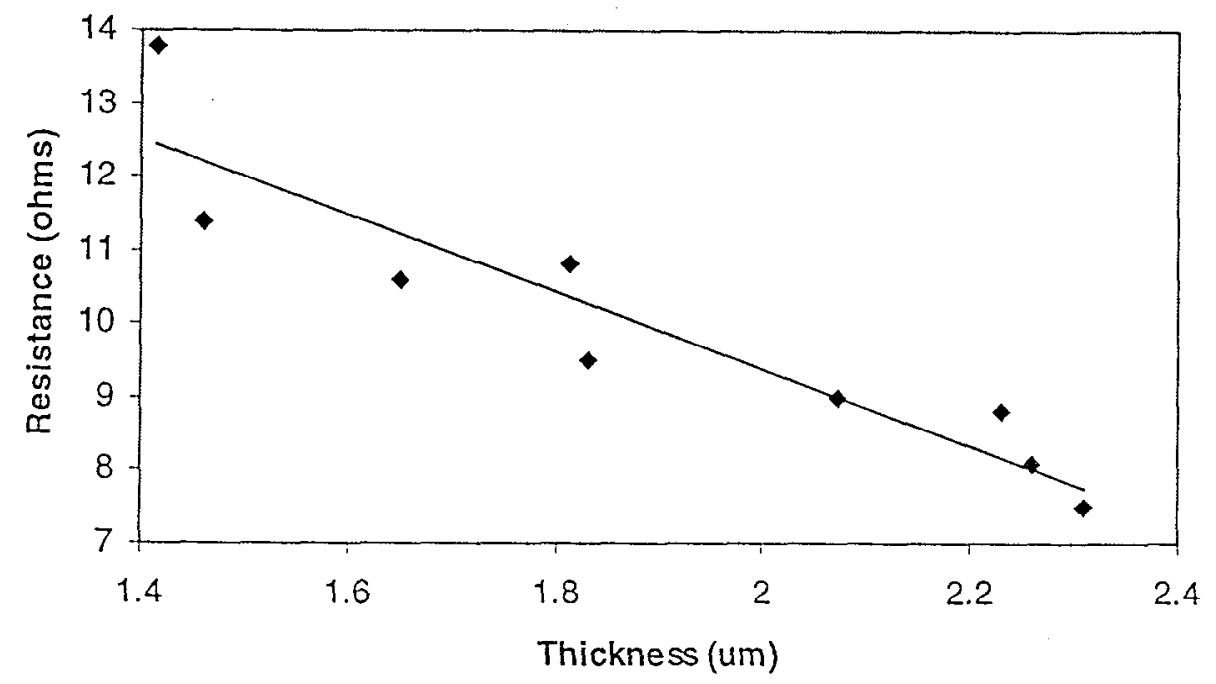

Figure 4.11: Ligament resistance versus thickness for the nine ligaments used in actuation testing.

The approximate martensite resistivity $\rho$ (see Equation 2-2) was found by an average of the products of thickness and resistance multiplied by the ratio of ligament width to length ( 400 $\mu \mathrm{m} / 4 \mathrm{~mm}=0.1$ ). The average thickness $\times$ resistance for the nine ligaments tested by actuation was $183 \mu \Omega \cdot \mathrm{cm}$. This approximation of the martensite resistivity relies on the assumption that all of the resistance of the ligament comes from the ligament legs, because of higher cross-sectional areas in other parts of the ligament structure. For instance, if the $1 \mathrm{~mm}$ length of the loading platform was included in the resistivity approximation, the ratio of width to length becomes 0.08 and the resistivity $\rho$ changes to $146 \mu \Omega \cdot \mathrm{cm}$. Obviously, the approximation of resistivity by this method has large sources of error, but gives a good idea of the magnitude or the resistivity of the material during martensite phase.

\subsubsection{Mechanical efficiency}

An extremely important property of any $\mu$-actuator is its mechanical efficiency: the ratio of output power to input power. In the case of these ligaments, the input power is the product of the voltage and the current required for actuation, and the output power is the product of the 
actuation force and displacement created by the ligaments divided by the time required for full actuation. For the ligament that was tested to create the typical results seen in previous sections, the power required was $230 \mathrm{~mW}$ and the time for full actuation was about $30 \mathrm{~ms}$. As seen in Figure 4.12 for this ligament, the work output (force $\times$ displacement) was a maximum at $62 \mu \mathrm{J}$. The power produced by the ligament was therefore $2.1 \mathrm{~mW}$ and the mechanical efficiency was $0.91 \%$. This is extremely low because of the high cross-sectional area of the ligaments, giving them a lower resistance and requiring more power for heating. The efficiency could be much greater with optimization of ligament geometry to cause more force and displacement but require less power.

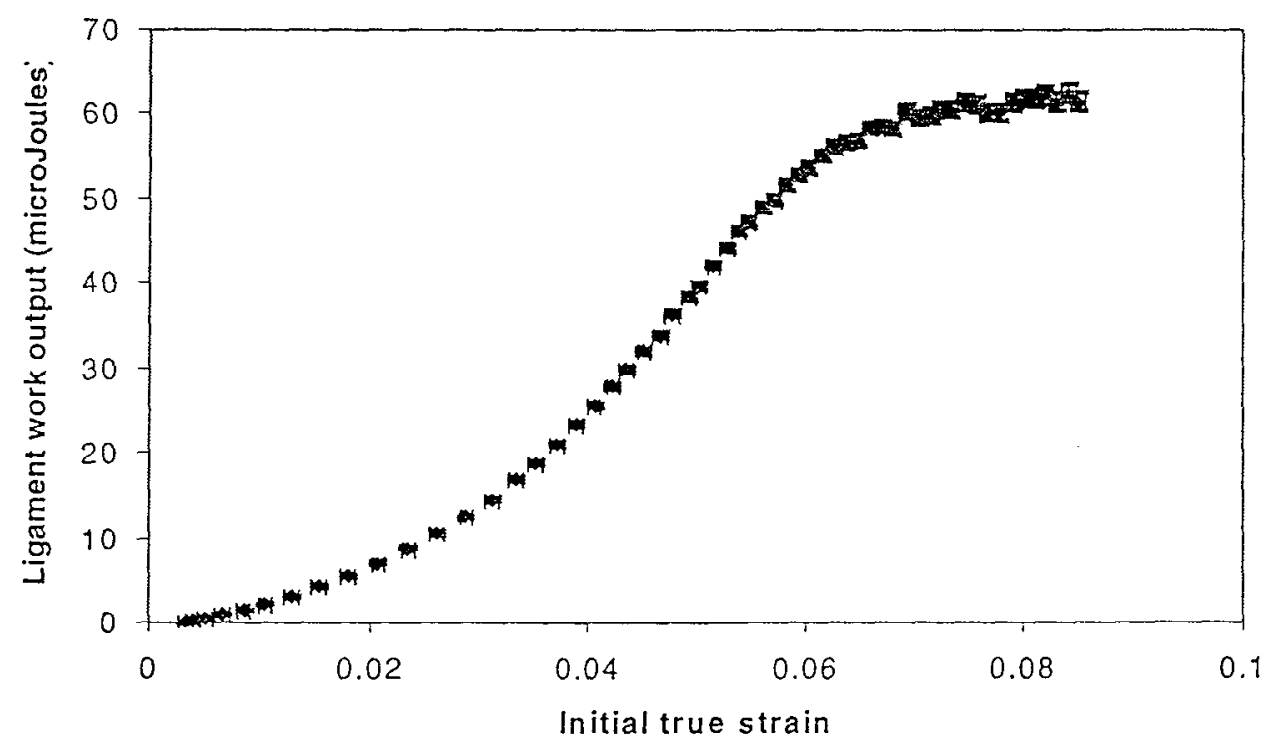

Figure 4.12: The actuation force $x$ displacement calculation gave the ligament work output during actuation tests.

\subsection{Fatigue tests}

\subsubsection{Heating and cooling times}

Fatigue testing was performed on ligaments with various initial true strains. Each ligament undergoing fatigue testing was loaded by advancing the micrometer to a certain displacement and slow cycling took place to allow measurement tuning. A $100 \mathrm{mHz}$ square wave was passed through the ligament and the $\mathrm{AC}$ voltage and $\mathrm{DC}$ offset were adjusted until the ligament experienced its maximum actuation. The DC offset was determined as just more than half of the peak-to-peak AC voltage, so that at the bottom of the square wave, a positive voltage was still being passed through the ligament and resistance could be measured by digital acquisition. 
After tuning the voltage, the frequency was increased to the maximum value that still allowed for full actuation of the ligaments. The duty cycle was also adjusted, because heating took less time than cooling. Through this tuning, frequencies of $3.5 \mathrm{~Hz}$ with $30 \%$ duty cycles were achieved, yielding total heating times (higher voltage) of $86 \mathrm{~ms}$ and total cooling times (lower voltage) of $200 \mathrm{~ms}$. While these times seem large, they were chosen to allow for full and complete actuation. Observation of displacement curves during fatigue cycling showed, however, that $90 \%$ of heating or cooling took place during the first $20 \%$ of the heating or cooling cycle. Thus, by reducing the need for full actuation to only $90 \%$ actuation, heating times change to 17 $\mathrm{ms}$ and cooling times decrease to $40 \mathrm{~ms}$. For active control, response times would be much less because the times listed here for full actuation are much greater than times needed for minimal actuation.

\subsubsection{Resistivity changes}

The current and voltage through the ligament were measurcd in real time by probing the voltage through the precision resistor in series with the ligament and the voltage drop across the ligament, respectively. Using these two system properties, the real time resistance of the ligament was measured to determine the resistivity changes during heating and during phase transformation from martensite to austenite. During cycling, a typical ligament had an initial resistance at room temperature of approximately $15 \Omega$. Upon heating, the resistance increased during the small periơd of time before the ligament began phase transformation. The maximum resistance right before phase transformation was about $16 \Omega$. As the phase transformation occurred, the ligament resistance dropped quickly to between 12 and $13 \Omega$ because of the lattice structure change to $\mathrm{BCC}$, where electrons could pass through the material more freely than during the orthorhombic phase. This drop in resistance held to the expected $20 \%$ drop in material resistivity from martensite to austenite, seen in previous literature [reference].

\subsubsection{Cycles to failure}

In order to determine the lifetime of actuators made from freestanding NiTiCu ligaments like the ones described in this paper, a cycle counter was implemented into fatigue measurements. Measuring the number of cycles to failure under different initial strains gave an idea of what applications this type of $\mu$-actuator could used for by illustrating how many times the actuator could be used before ligament fracture occurred and the device was rendered useless. Figure 4.13 displays the cycles to failure for three ligaments tested under a useful range of initial strains. Through linear interpolation, the behavior of $\mathrm{NiTiCu}$ ligaments could be approximated and the number of cycles to failure under different initial strains could be inferred. The plot in this figure 
was linearized by the logarithmic scale of the $x$-axis to help with approximations. The trendline connecting the three data points approximates the maximum number of cycles achievable under small strains but due to a lack of data the validity of this linear solution could not be proven. If the trendline was accurate, 1 million cycles would be impossible with the ligaments, which is highly unlikely. It is more likely in this case to assume that as smaller initial strains were placed on a ligament, the cycles to failure would grow asymptotically and with recoverable strains at some value less than $0.4 \%$, the ligaments would reach billions of cycles and could be assumed to last forever. At the other end of the spectrum, it is seen by the slope of the cycles to failure curve ( $1 \%$ recoverable strain per order of magnitude of cycles) that the maximum recoverable strain of $3.0 \%$ seen in Figure 4.8 would last less than 1000 cycles before fracturing.

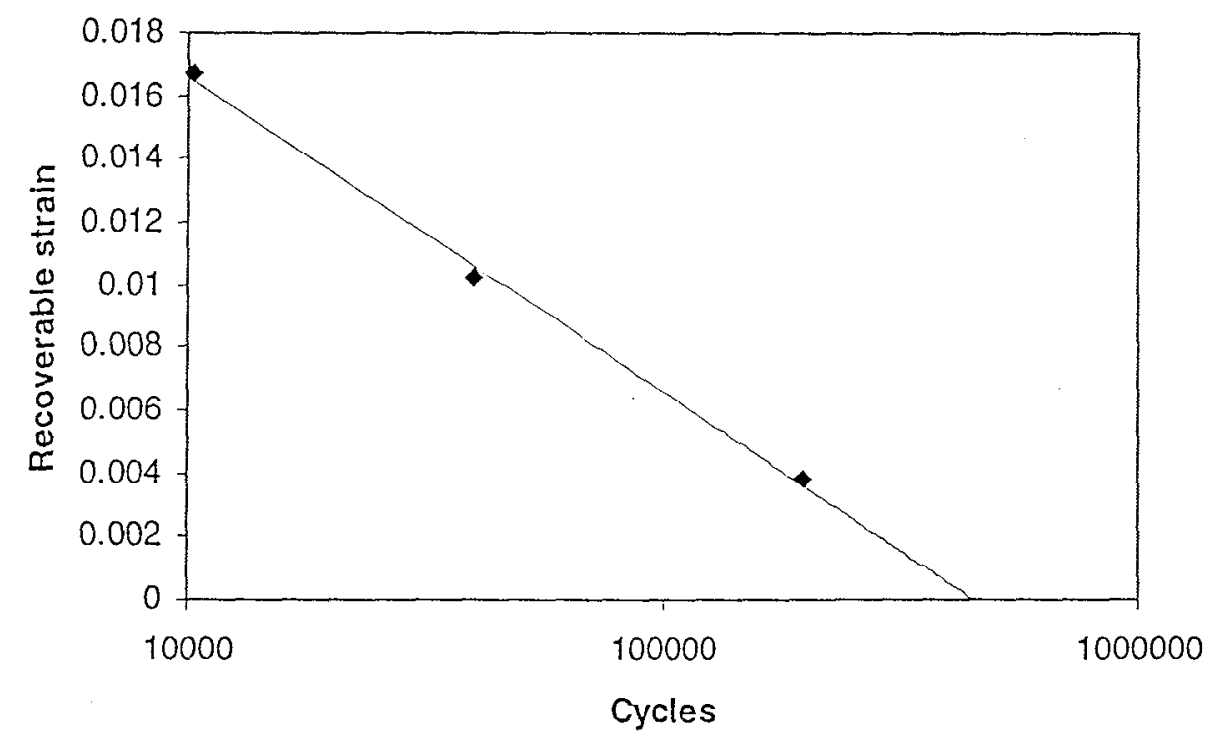

Figure 4.13: Cycles to failure for three ligaments were found through fatigue testing and a logarithmic trendline was fitted to the data to aid in approximating data for design purposes.

Data like this is pertinent in the design of $\mu$-actuators because designers can determine how long devices will last and whether or not they can be used in applications with active control. For example, devices employing freestanding NiTiCu ligaments would work extremely well in medical applications where actuation is used only a few times and a device is thrown away. In a case like this, the MEMS device could be designed to operate with the maximum recoverable stress and strain possible without worry of device failure. On the other hand, if large actuation was necessary over millions or billions of cycles, devices employing SMA actuation would need to be painstakingly designed to insure that devices would not fail or need frequent replacement. This is not to say that large actuation and long life cannol coexist with a NiTiCu actuator; it is likely that thicker films would greatly increase actuator lifetime by decreasing the stress in 
freestanding ligaments. The effect of film thickness on ligament life was not investigated in this study because all deposited films were approximately $2 \mu \mathrm{m}$ thick to keep other measurements consistent.

\subsubsection{Reduced shape memory recovery with cycling}

During the procession of cycling, a slight reduction in the recovered strain produced by the ligaments was noticed. This behavior was noticed in all three of the ligaments tested by cycling, pointing out the occurrence of some shape memory fatigue before fracture. In each case; the fatigue brought the recovered strain to a value less than $25 \%$ away from the original recovered strain at the point of fracture, with more fatigue found in ligaments that underwent more cycles.

Figure 4.14 shows this reduction in shape memory recovery for the three ligaments by comparing the recovered strain measured every 100 cycles to the number of accumulated cycles.

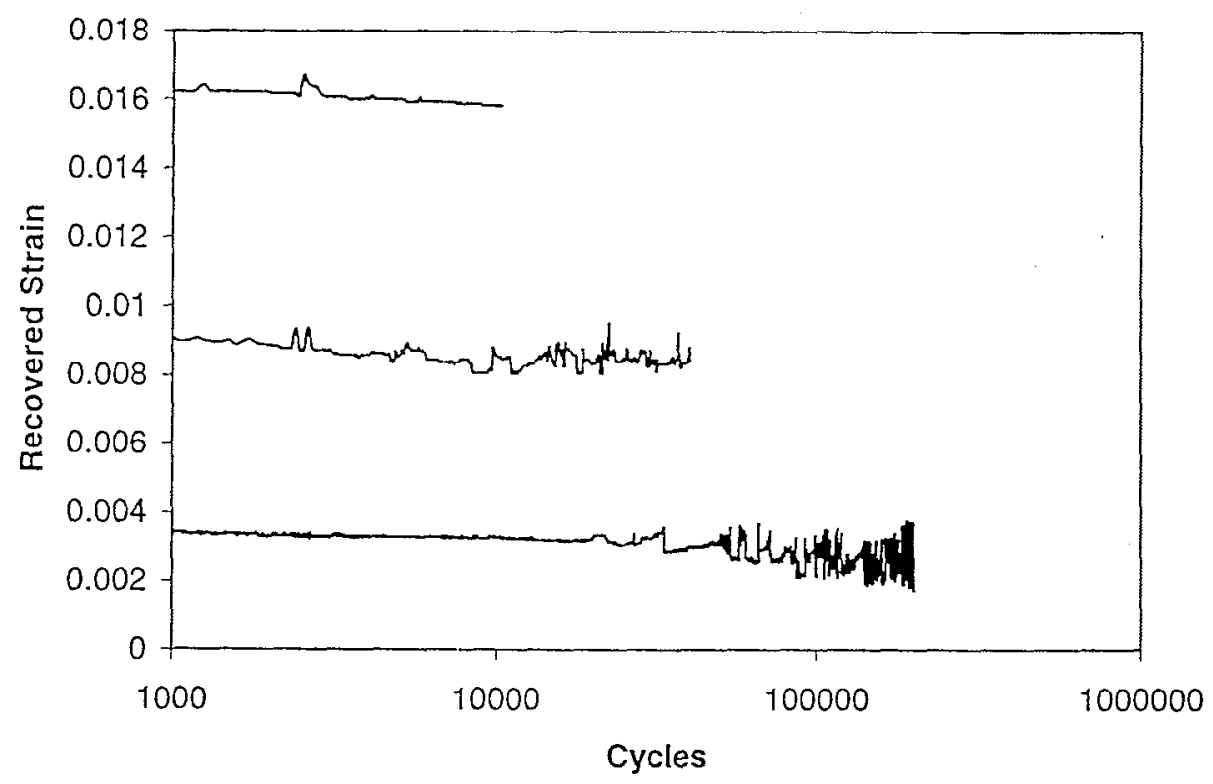

Figure 4.14: The recovered strain found every 100 cycles illustrates the slight loss of shape memory recovery throughout a ligament's lifetime.

The data from these tests also illustrates some inconsistency in shape memory effect encountered as the number of cycles increased. With smaller recovered strains, the inconsistent behavior occurred later in cycling, proving that this phenomenon was stress dependent. The two ligaments with lower recovered strain had rather consistent, linear behavior before the inconsistency began, while the highly strained ligament fractured without ever entering an inconsistent regime. 
By averaging out the inconsisient regime of the ligament with the lowest recovered strain, the plot in Figure 4.15 was created. Every 200 data points of the plot in Figure 4.14 were averaged and their values were plotted against the number of accumulated cycles to give a better idea of the trend in recovered strain in the ligament. Notice should be taken in the comparison of these two figires because Figure 4.14 has a logarithmic $x$-axis, whereas Figure 4.15 has a linear axis. The decrease in shape memory recovery is made clearer by the averaged data by expunging the inconsistencies that made the data unreadable. The recovered strain of $0.33 \%$ seen at the onset of cycling diminished to a minimum of $0.24 \%$ by 150,000 cycles and then increased back to $0.27 \%$ before fracturing. The sporadic strain behavior of the ligament at high cycles, including the increase in recoverable strain at very high cycles, can be explained by fatigue stiffening of the material with cycling. As cycling would begin, the ligament would experience less and less strain recovery due to the formation of permanent dislocations, shifting the austenite stress-strain curve to the right and causing the difference in initial and actuated strain to be less. As the cycling continued, however, stiffening of the material caused a slight shift of the austenite curve back toward the initial curve and the difference between strains grew again. The inconsistent behavior of the ligament during stiffening is understandable because the mechanisms of stiffening are very nonlinear and random, causing the ligament to experience immense fluctuations in behavior.

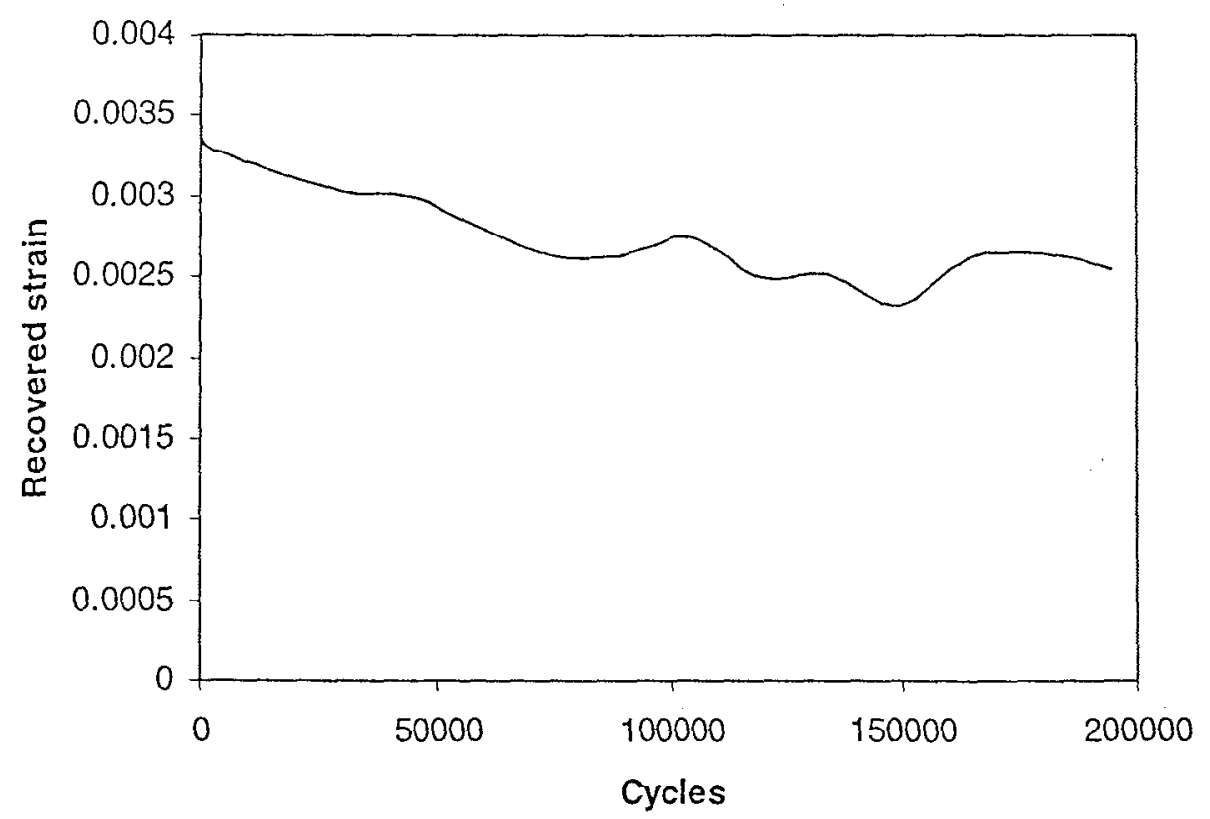

Figure 4.15: Averaged data gives a better indication of the behavioral trend of a ligament undergoing cycling under the lowest strains used in fatigue testing. 


\subsubsection{Maximum displacements and forces achievable for long life devices}

While the recoverable strain may seem small for the ligament that reached 200 kcycles, the actuation deflections and forces were actually quite large for the purposes of implementation into MEMS devices. Most MEMS actuators produce deflections on the order of $10 \mu \mathrm{m}$ and forces in the $\mu \mathrm{N}$ range. However, since the ligaments tested here were larger than most MEMS devices, with mm scale dimensions, they could produce actuations of up to $90 \mu \mathrm{m}$ (see Figure 4.16) and maximum forces of $25 \mathrm{mN}$ (Figure 4.17). The maximum forces in this data are the total compression force in the DVRT spring during the actuated state of the ligament. This data proves that long lasting devices can be made that provide enough motion and force for a wide variety of applications, even though the recoverable strains were much smaller than what was possible with these actuators. The ligament that created the plots seen below still experienced inconsistency late in its life, but for nearly the first $50 \mathrm{kcycles}$, the behavior was unwavering.

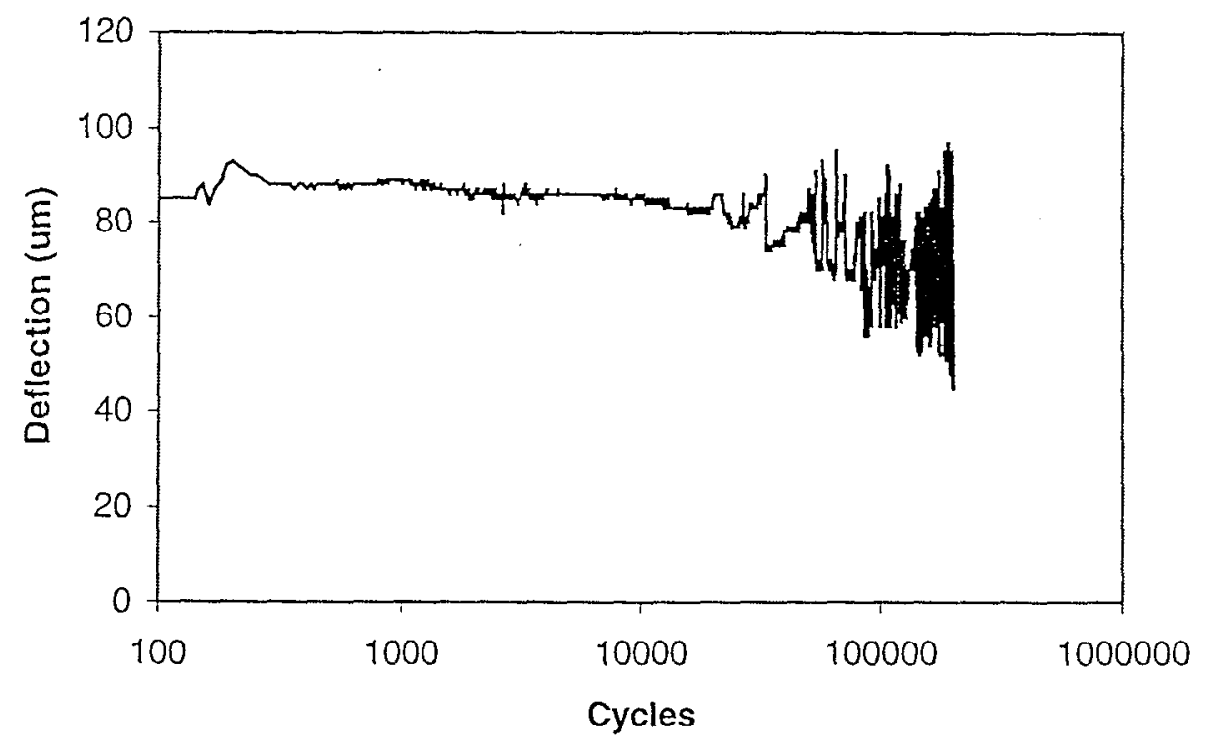

Figure 4.16: Actuation deflection of the ligament with smallest recovered strain that underwent fatigue testing showed much higher deflections than those accompanying common MEMS devices.

It should also be noticed that in Figure 4.17 the maximum force produced in the DVRT spring against the ligament was more consistent than the recovered strain or actuation deflection measurements seen in Figure 4.14 and 4.16. This is due to the fact that the initial displacement was not considered in these measurements, and therefore fluctuations in the martensitic behavior of the material were not accounted for in the data seen in Figure 4.17. The maximum force produced is a beneficial measurement because it is the amount of force the ligament can produce to close off a valve or create actuation against a bias spring designed into a MEMS device. Shape 
memory alloys are proven to be an effective source of actuation because of these achievable forces and displacements.

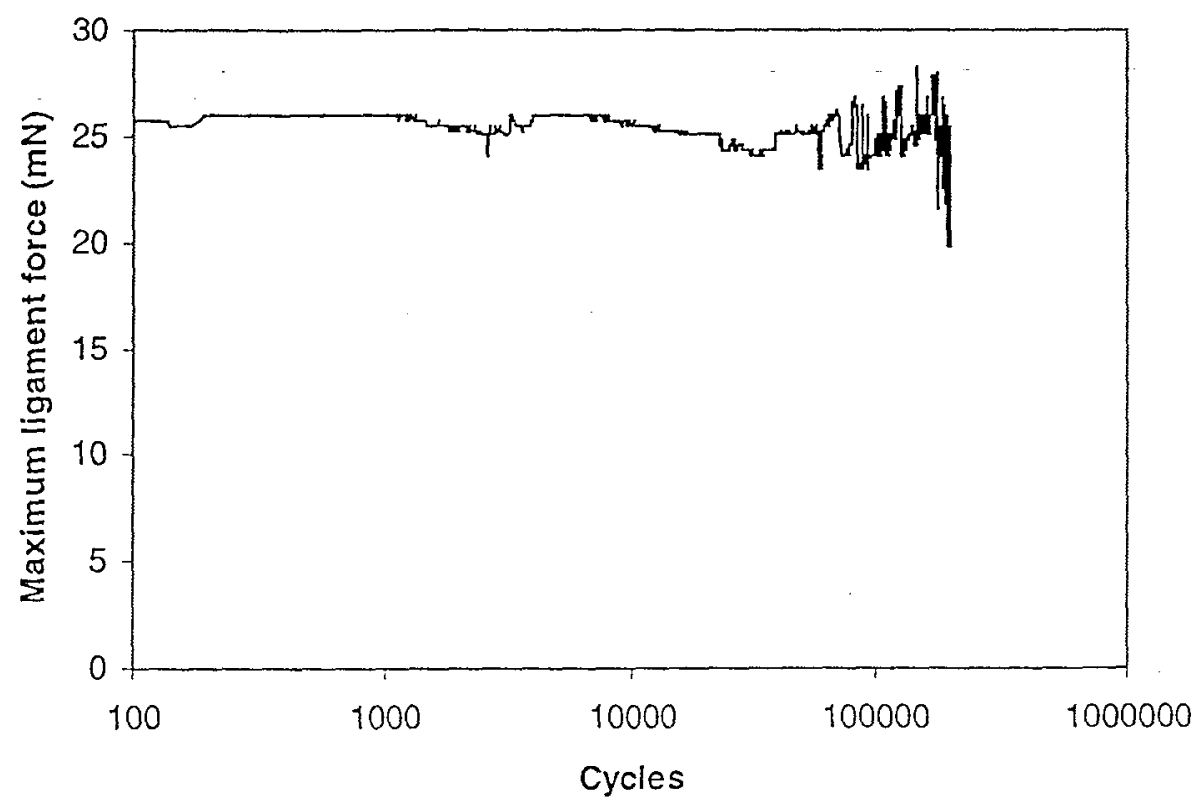

Figure 4.17: The maximum force produced by the longest lasting ligament that underwent fatigue testing. 


\section{Chapter 5: Conclusions}

\subsection{Young's modulus and stress-induced twins}

Thorough examination of the stress-strain curves in Figures 4.4-4.6 indicates that no clear Young's modulus or plate modulus can be calculated from the data. The plausible explanation that increasing the power through ligaments would increase the volume fraction of twins recovered is one reason that these curves do not give the clear linear relation between stress and strain in the austenite curve. For the purposes of these experiments, characteristic functions were devised and their curves were plotted alongside the stress-strain data for martensite and austenite trials. The best-fit function for the austenite phase was a power function and for the martensite phase was a quadratic function. While no clear modulus determination was made, these functions would easily aid in the design of MEMS devices utilizing NiTiCu films with the same composition and fabrication methods described in Section 3 of this paper.

The functions seen in Equations 4-1 and 4-2 are interpretations of data where full recovery was not occurring. The Clausius-Clapeyron equation states that more recovery would occur if more power was applied at high stresses, but this possibility was not tested and is simply theoretical at this point. However, the likelihood of stress-induced twinning is corroborated by the data of Figure 4.5. Ligaments that were tested while purely in martensite or austenite phase seemed to show that more stress recovery was possible than the ligaments that underwent testing where actuation occurred back and forth between the two phases. When ligaments had to increase their tensile stress while undergoing a phase transformation, less recovery was seen than when they had been in the austenite phase during the entire test. The energy balance dictating this behavior is explained by Clausius-Clapeyron; therefore, by increasing the power through the ligament either during actuation or steady state austenite, a higher volume fraction of twins in the material would be recovered. In the actuation case, they would recover from the highly twinned martensite into the austenite phase; and in the static case, there would simply be less stressinduced twinning.

One possible method to remedy the situation of stress-induced twins is active feedback control of the power through the ligament. Increases of ligament power until maximum actuation is achieved would also maximize the volume fraction of twins recovered. While it cannot be assumed that all twins would recover to $\mathrm{BCC}$ by increasing the power to this optimum value, there would at least be more twin recovery and likely a more linear stress-strain curve for the austenite phase. 


\subsection{Cycles to failure}

During fatigue testing of ligaments, the highest number of cycles to failure was 200,000 for the ligament under the lightest load. Other ligaments, under much higher loads, exhibited cycles to failure of 10,000 and 40,000 . High deflections of $90 \mu \mathrm{m}$ and forces of $25 \mathrm{mN}$ were still possible with the ligament under lightest load. Because the forces and deflections under the lightest load were this high, it is possible that millions or billions of cycles were achievable with these ligaments under lighter loads. Actuators of this type could therefore be useful in a wide variety of applications, from automobile parts with nearly infinite lifetimes to medical devices with only a few necessary cycles. The actuators for each application would have to be designed with the cycles to failure in mind, such that the automobile actuators would be under very low stresses whereas the medical actuators could utilize the higher forces and deflections possible with the freestanding SMA films.

\subsection{Design method for SMA $\mu$-actuators}

With a comprehensive testing instrument like the one described in this paper, the design of SMA microactuators is greatly simplified. With accurate material property measurements, design entails merely coming up with the constitutive equations for the device in question and correlating those equations with the stress-strain curves found by testing ligaments with the same composition and fabrication methods. Fitting equations to new stress-strain curves similar to those seen in Section 4.3.2 allows analytic solutions to the design problems associated with SMA films.

An example of this design method is illustrated by looking at the testing instrument used to qualify the films. The DVRT spring constant dictates how much force is placed on the films based on how much deflection they produce. Therefore, the stress and the strain in the film are directly correlated by the linear spring constant and the geometry of the ligament. With this in mind, the stress-strain equations in Equation 4.1-4.2 could be applied to determine how much displacement and force the ligaments could create under specified loading conditions.

The application of this predictive method to ligaments with different geometry should produce results that, within a slight degree of error, would state how much force and deflection they could produce, given specific loading conditions. By stretching this application to morc than just ligaments, it is seen how any SMA microactuator could be designed using this model after the alloy is initially qualified.

The benefits of this instrument do not stop at qualifying films of certain composition to aid in the design of microdevices. It can also qualify the devices after they are fabricated by 
directly measuring the forces and displacements they achieve while they are loaded with appropriate forces or pressures. An instrument setup like this could also be used in-line for device qualification, to allow for quality control during a MEMS manufacturing process by checking one or several devices per wafer for forces, deflections, power requirements, and calibration errors all at the same time and in just a few seconds.

\subsection{Future work}

The full characterization of NiTiCu films for use in the design of MEMS devices is far from complete. Additional steps toward understanding the mechanical behavior of these films have been taken with the studies described in this paper, but more work can be done along the same lines and with the same testing instrument to obtain a relatively comprehensive behavioral model.

One future goal is to correlate the effects seen in the data presented here and the Clausius-Clapeyron effect. To understand the power requirements and energy balance nceded to fully transform martensitic twins to austenite $\mathrm{BCC}$ crystals, active control should be implemented to achieve the greatest possible actuation and twin recovery under all loading conditions. The performance of experiments involving this type of control system will likely result in stress-strain curves that are shifted to include greater austenitic stress at a particular strain and have a linear regine from which the plate modulus (and subsequently the Young's modulus) can be inferred.

Future experiments should involve ligaments with dissimilar geometry to find out if the stress-strain curves hold for the material in all orientations. Smaller ligaments should be fabricated to determine forces and displacements possible with MEMS-scale actuators, rather than the millimeter-scale actuators studied in this paper. Through correlation of the data for a broad range of actuator size and geometry, design rules can be created for the use of NiTiCu films in MEMS devices.

Further testing should also be conducted using fatigue cycling and investigation of fatigue specimens. Lower loads should be placed on fatigue specimens to determine if cycling can reach into the millions or billions, thus providing nearly infinite lifetimes. Analysis of the twin structure of fatigued films through tunneling electron microscopy will elucidate the effects of cycling on the martensite structure of the SMA. Investigation can also be made into the modes of fracture of the fatigue specimens by examining the fractured ends under scanning or tunneling electron microscopes. These processes will determine the amount of necking that likely occurred before fracture and if the fracture grew from crack propagation due to non-uniform surface texture of the ligaments. 


\section{Appendix A: Uncertainty analysis}

The validity of any experiment to determine material propertics rclies heavily on the sources of error encountered in the tests and the propagation of error throughout the involved calculations. Uncertainty analysis was performed on each equation under Section 2.5 so that data plots could be made with appropriate error bars. This analysis was necessary to prove that data found in these tests was not overcome by sources of experimental error, because resolution issues were encountered with the measurement devices. Sources of error and values of uncertainty are outlined in Table A.1. Notation for absolute uncertainty of a variable $X$ in the following table is $\delta X$ for absolute uncertainty and $\delta X X$ for percent uncertainty. Depending upon how the error value was determined, absolute or percent uncertainties were employed.

TABLE A.1: Experimental error values for ligament deflection experiments.

\begin{tabular}{|l|c|c|c|}
\hline Measurement description & Notation & Error Notation & Error Value \\
\hline Length of one leg of ligament & $L$ & $\delta L$ & $\pm 10 \mu \mathrm{m}$ \\
\hline Width of gauge section of ligament & $w$ & $\delta w$ & $\pm 5.0 \mu \mathrm{m}$ \\
\hline Ligament thickness & $h$ & $\delta /$ & $\pm 0.1 \mu \mathrm{m}$ \\
\hline DVRT spring constant & $k$ & $\delta$ & $\pm 0.002 \mathrm{~N} / \mathrm{mm}$ \\
\hline Gap width between fractured ends & $e$ & $\delta e / e$ & $\pm 5.0 \%$ \\
\hline Micrometer displacement & $d_{m}$ & $\delta d_{m}$ & $\pm 1.0 \mu \mathrm{m}$ \\
\hline DVRT compression in either state & $d_{1.2}$ & $\delta d_{1.2}$ & $\pm 1.5 \mu \mathrm{m}$ \\
\hline
\end{tabular}

These values were used to propagate the error through the equations of Section 2.5, using the propagation rules that follow.

For addition and subtraction, error propagation involves the geometric mean of the absolute uncertainties:

$$
\begin{aligned}
q & =a+b-c \\
\delta q & =\sqrt{\delta a^{2}+\delta b^{2}+\delta c^{2}}
\end{aligned}
$$

For multiplication and division, propagation comes from the geometric mean of percent uncertainties:

$$
\begin{aligned}
q & =a b c \\
\frac{\delta q}{q} & =\sqrt{\left(\frac{\delta a}{a}\right)^{2}+\left(\frac{\delta b}{b}\right)^{2}+\left(\frac{\delta c}{c}\right)^{2}}
\end{aligned}
$$


For powers: $\begin{aligned} q & =x^{n} \\ \frac{\delta q}{q} & =n \frac{\delta x}{x} \\ q & =f(x) \\ \text { And for functions: } \quad \delta q & =\frac{d q}{d x} \delta x\end{aligned}$

Using these general rules, the following uncertainty equations were generated for force, stress, strain, and work output, with the derivations for force and stress shown as examples.

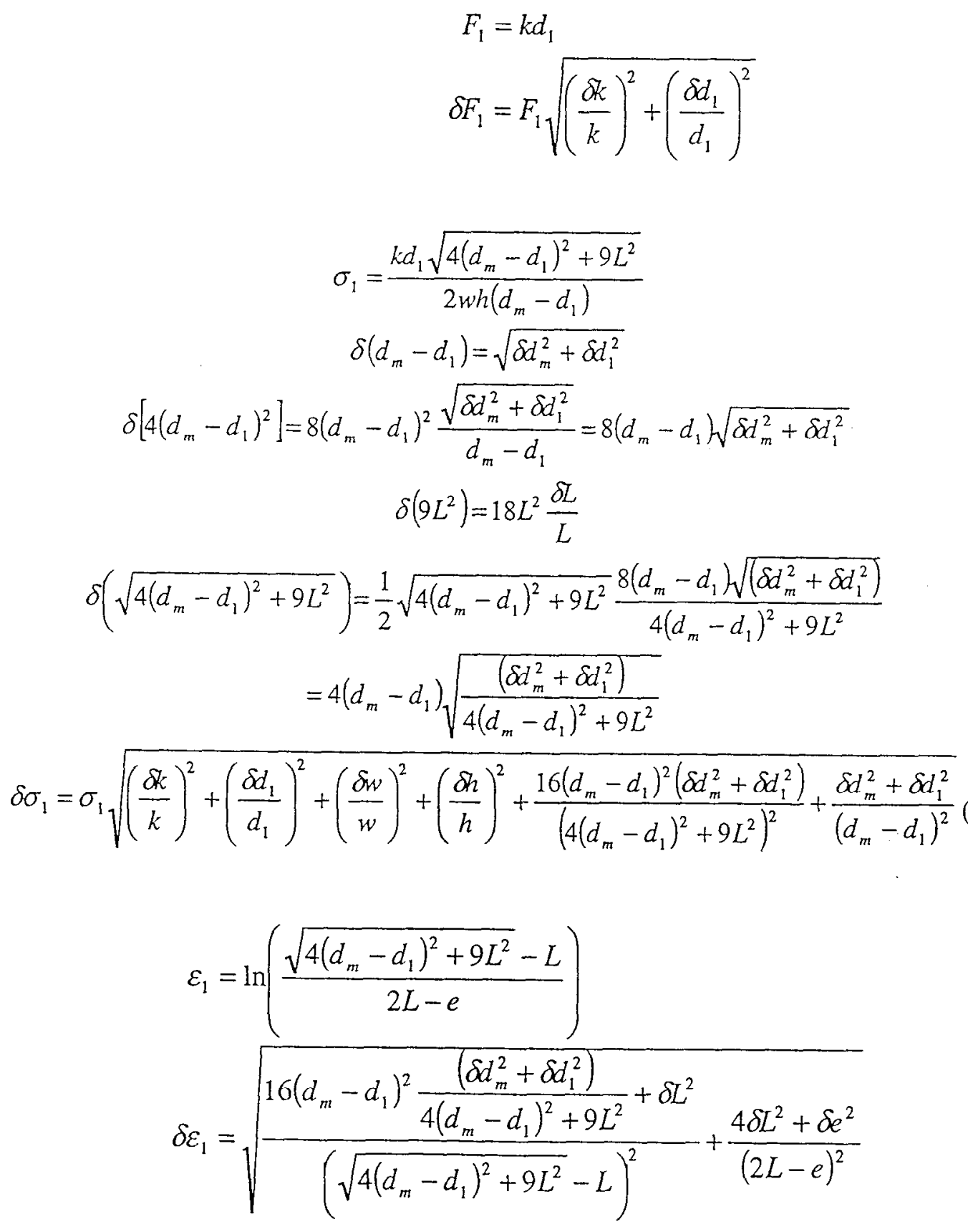

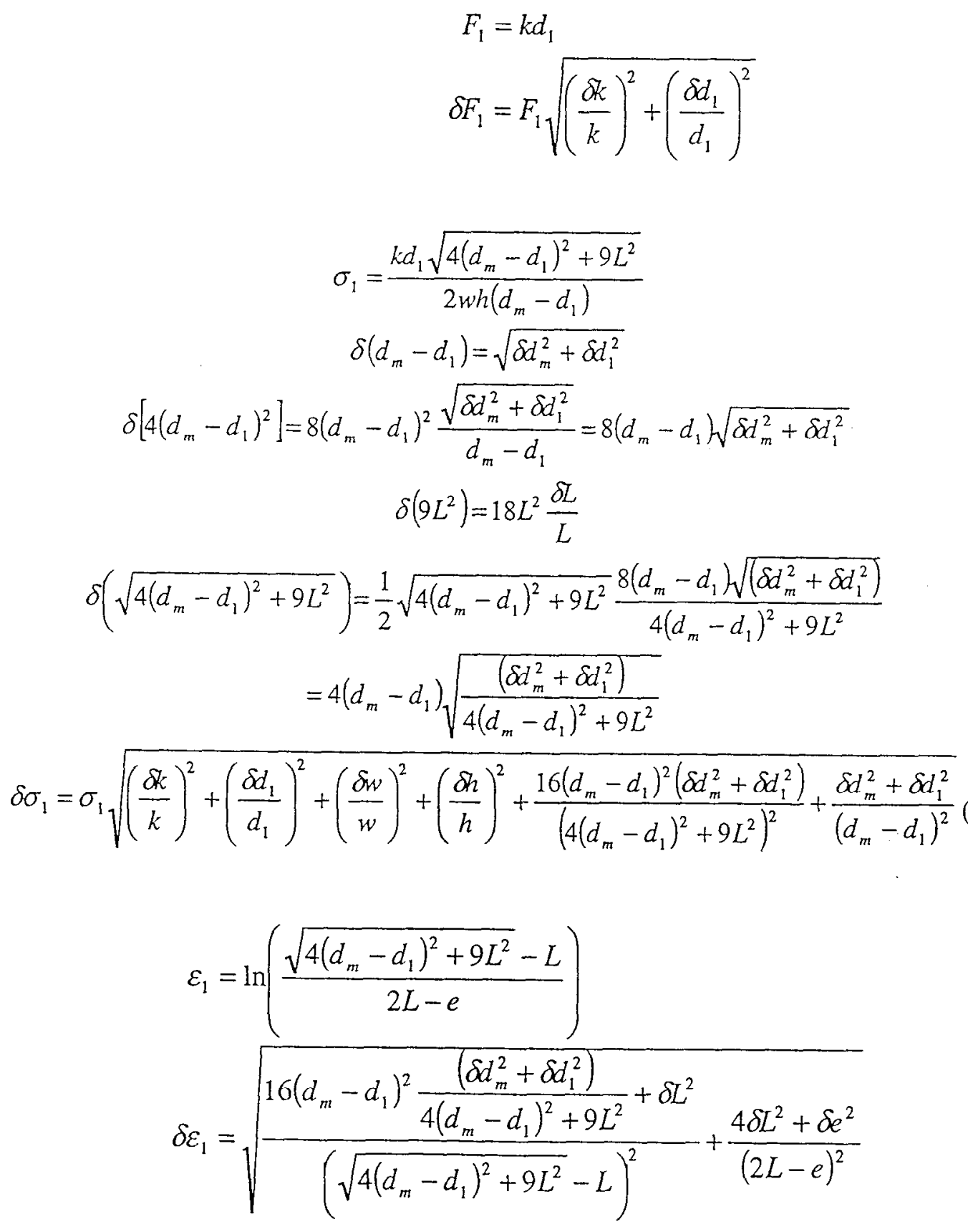

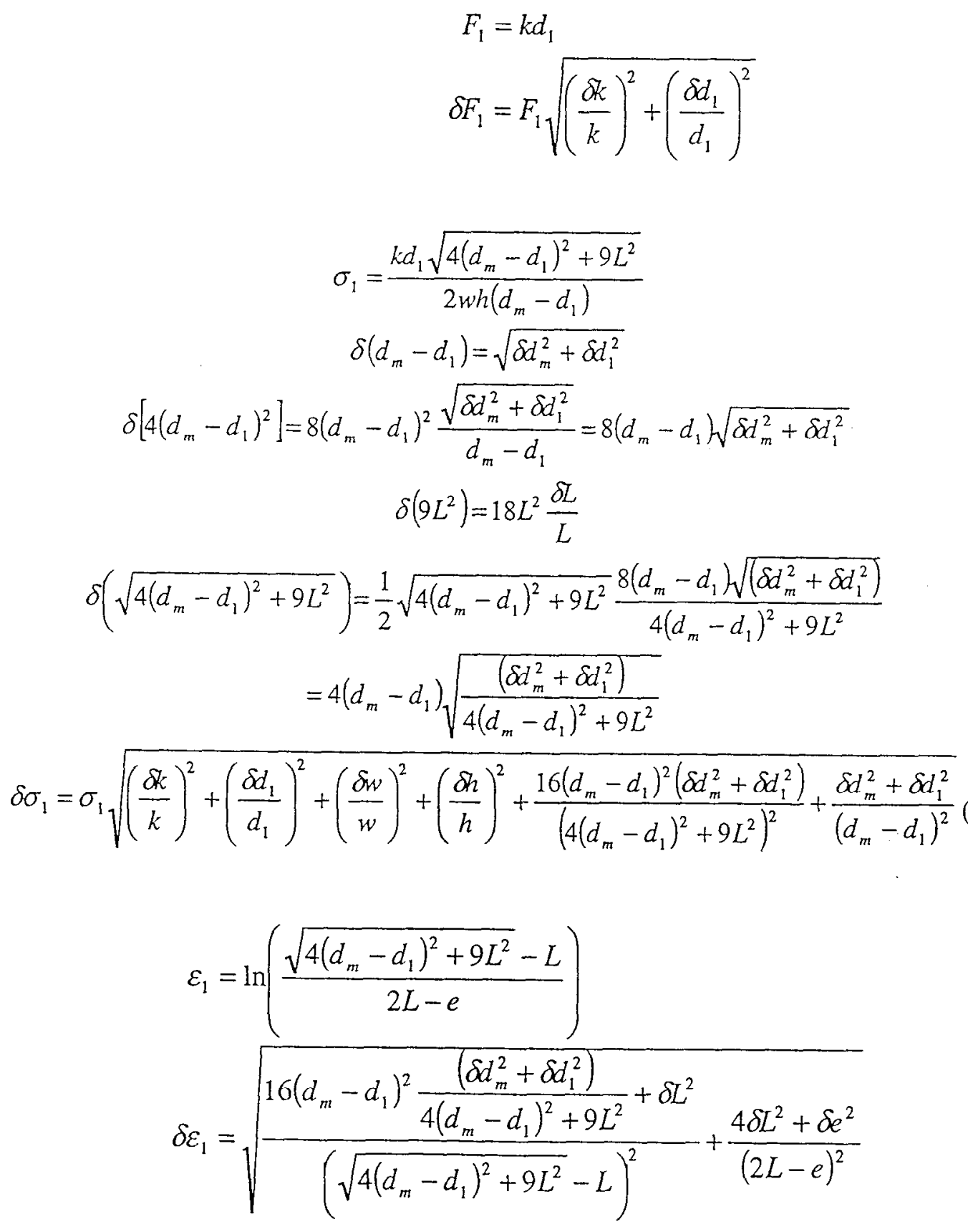

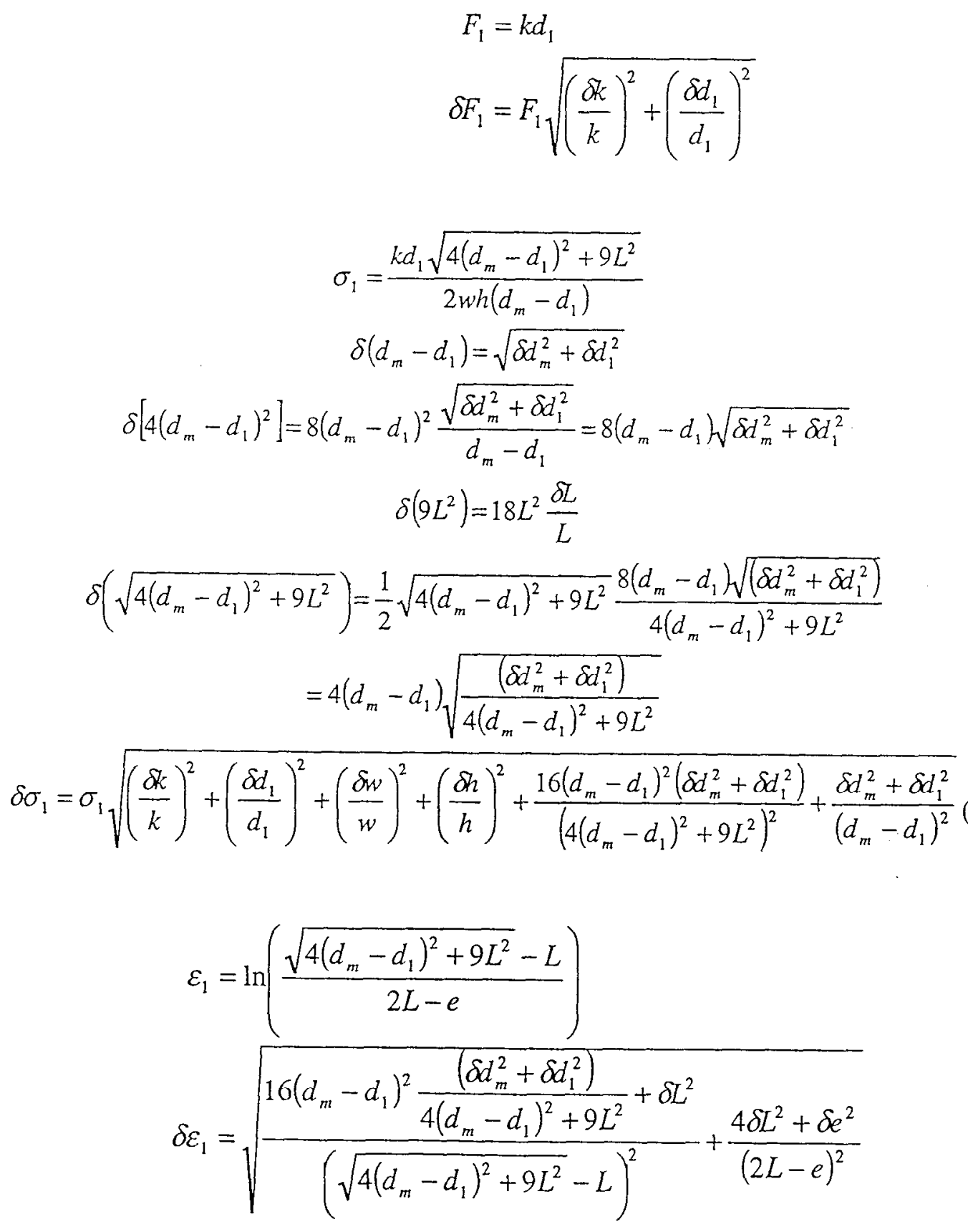

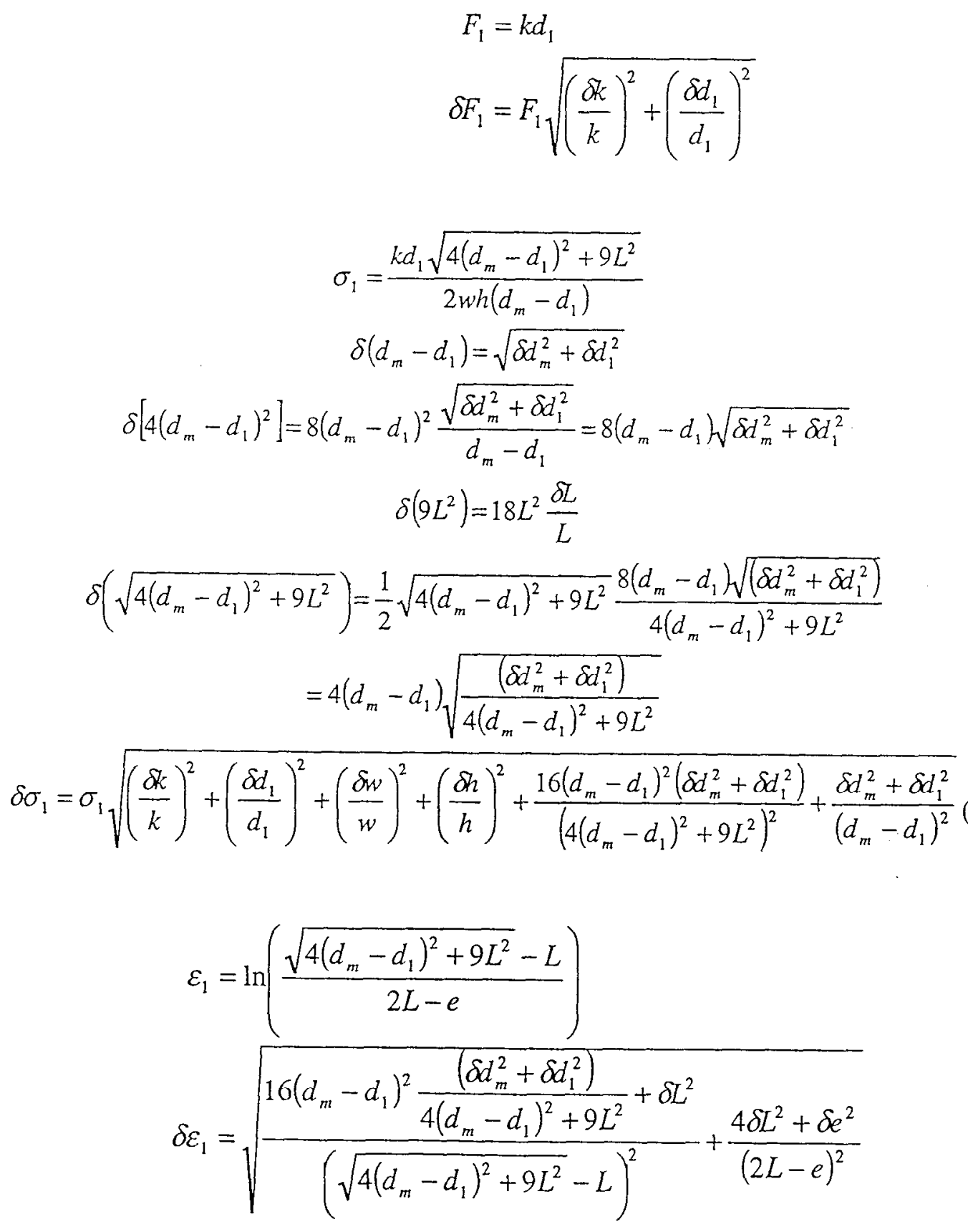

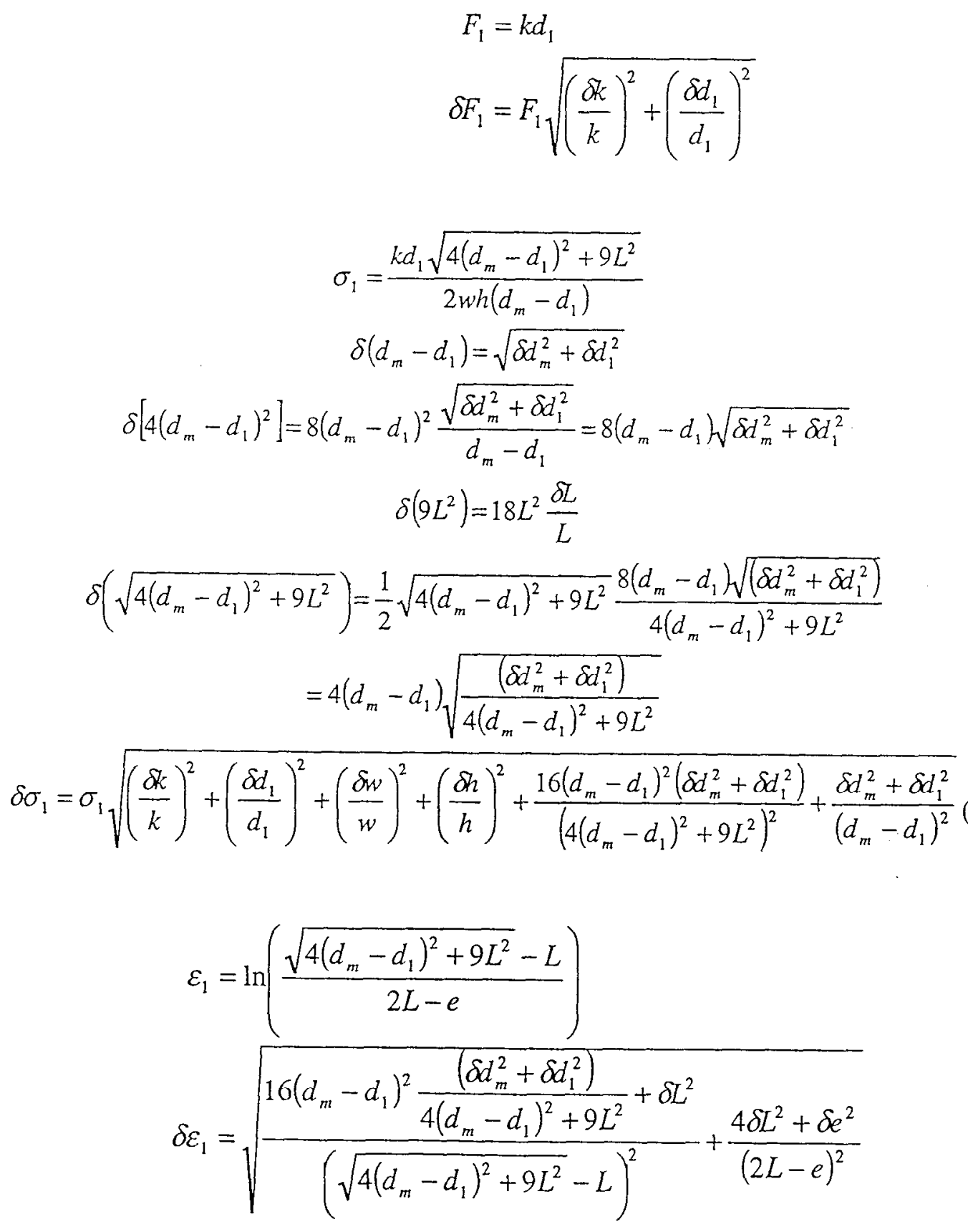

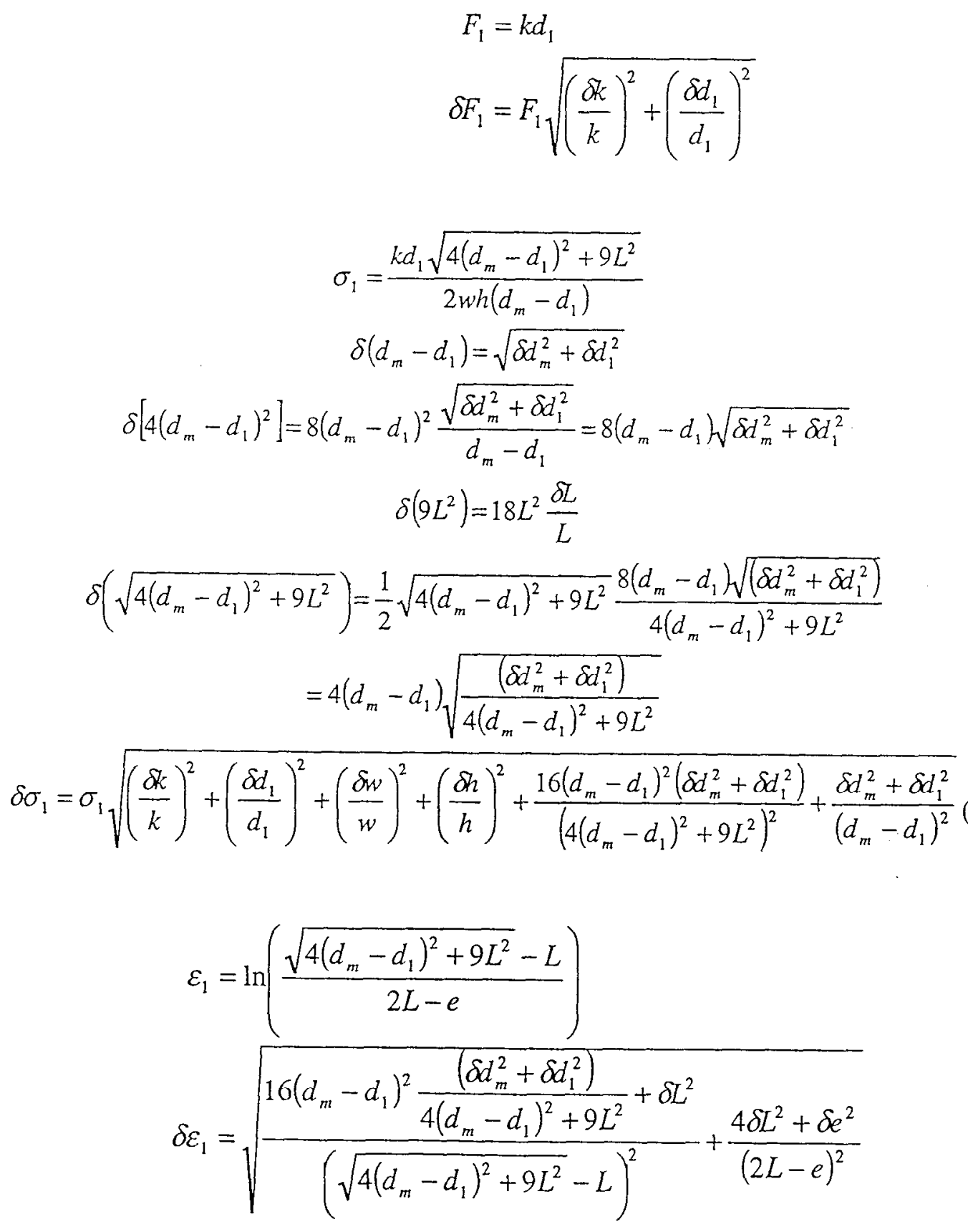

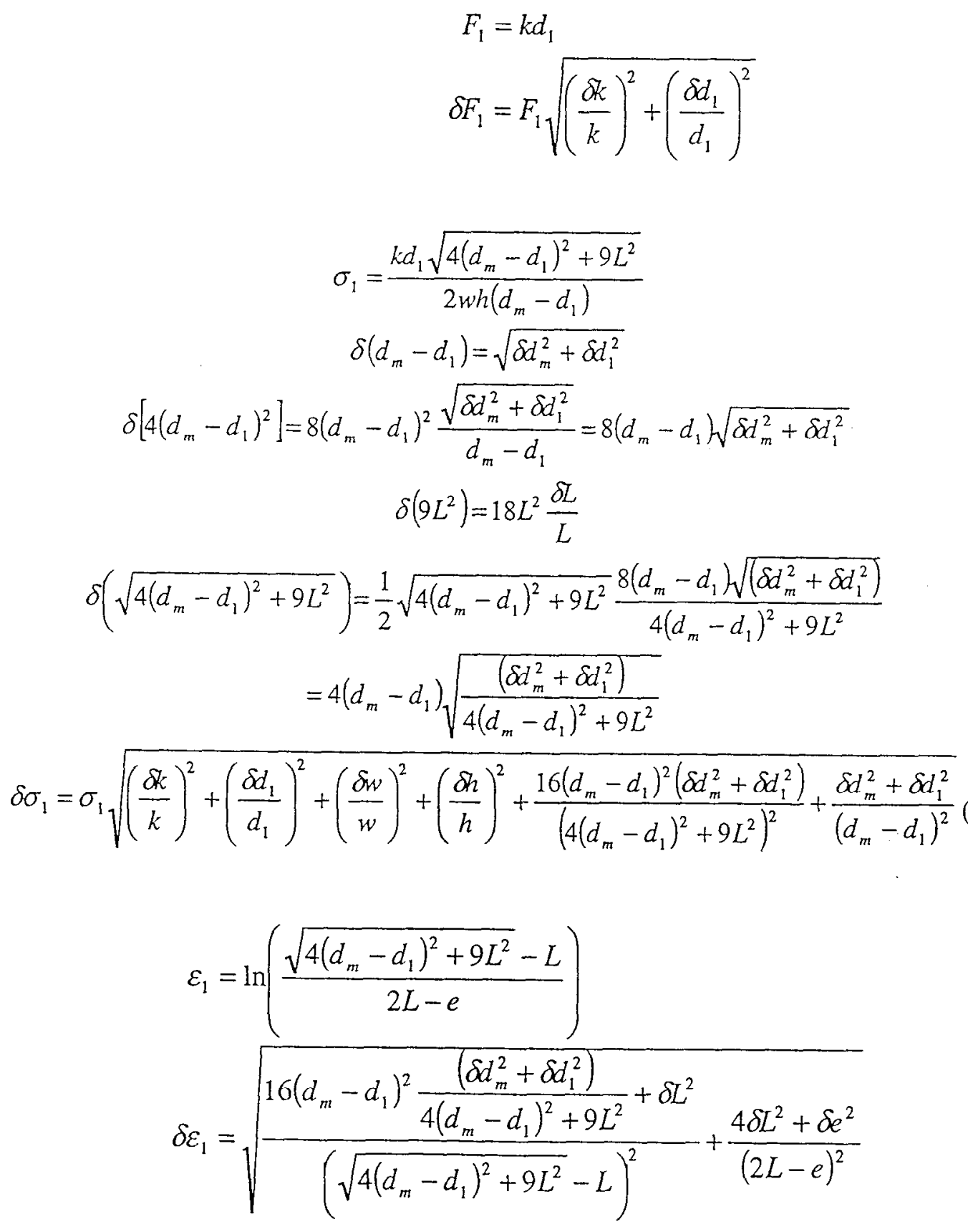

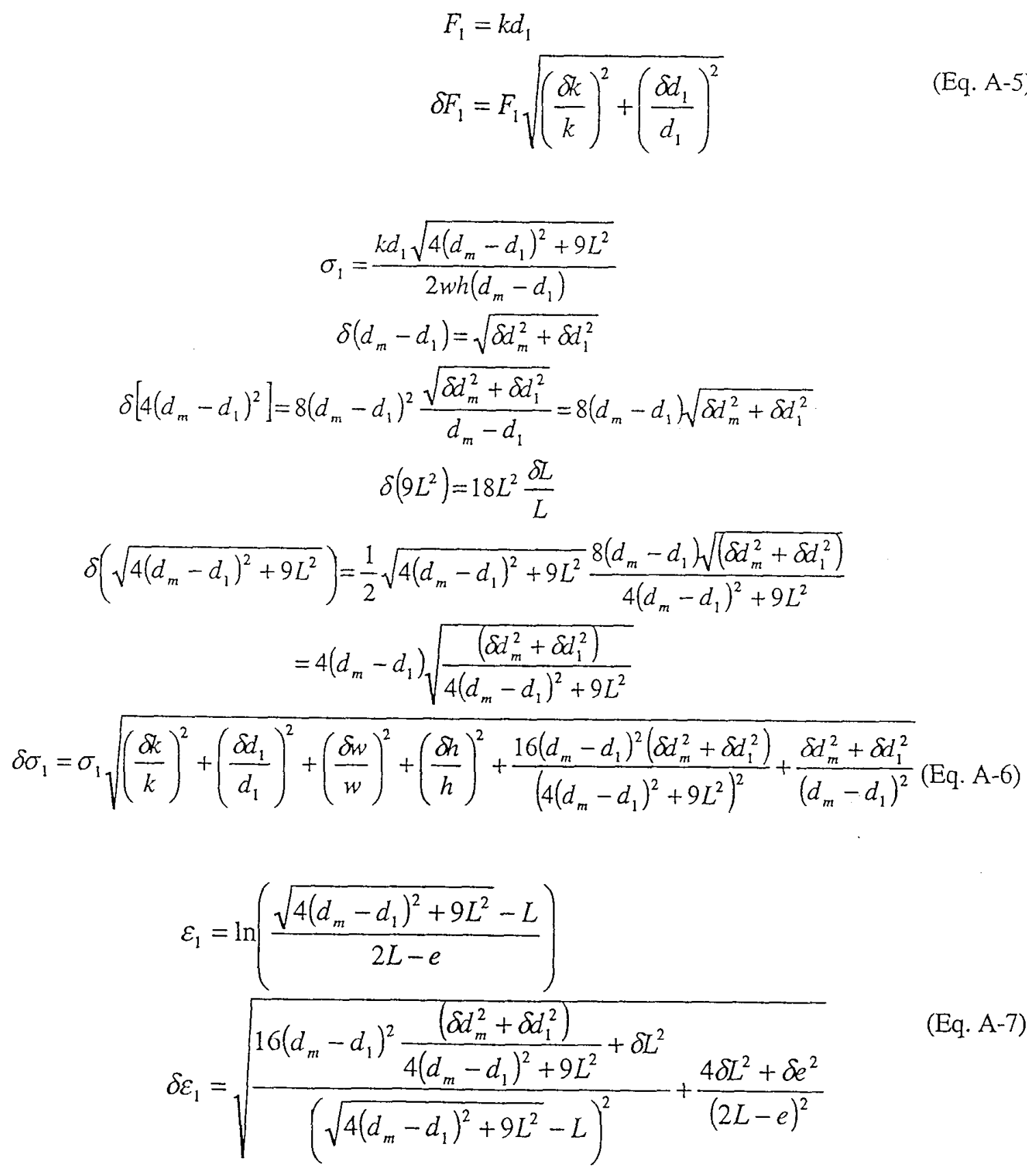

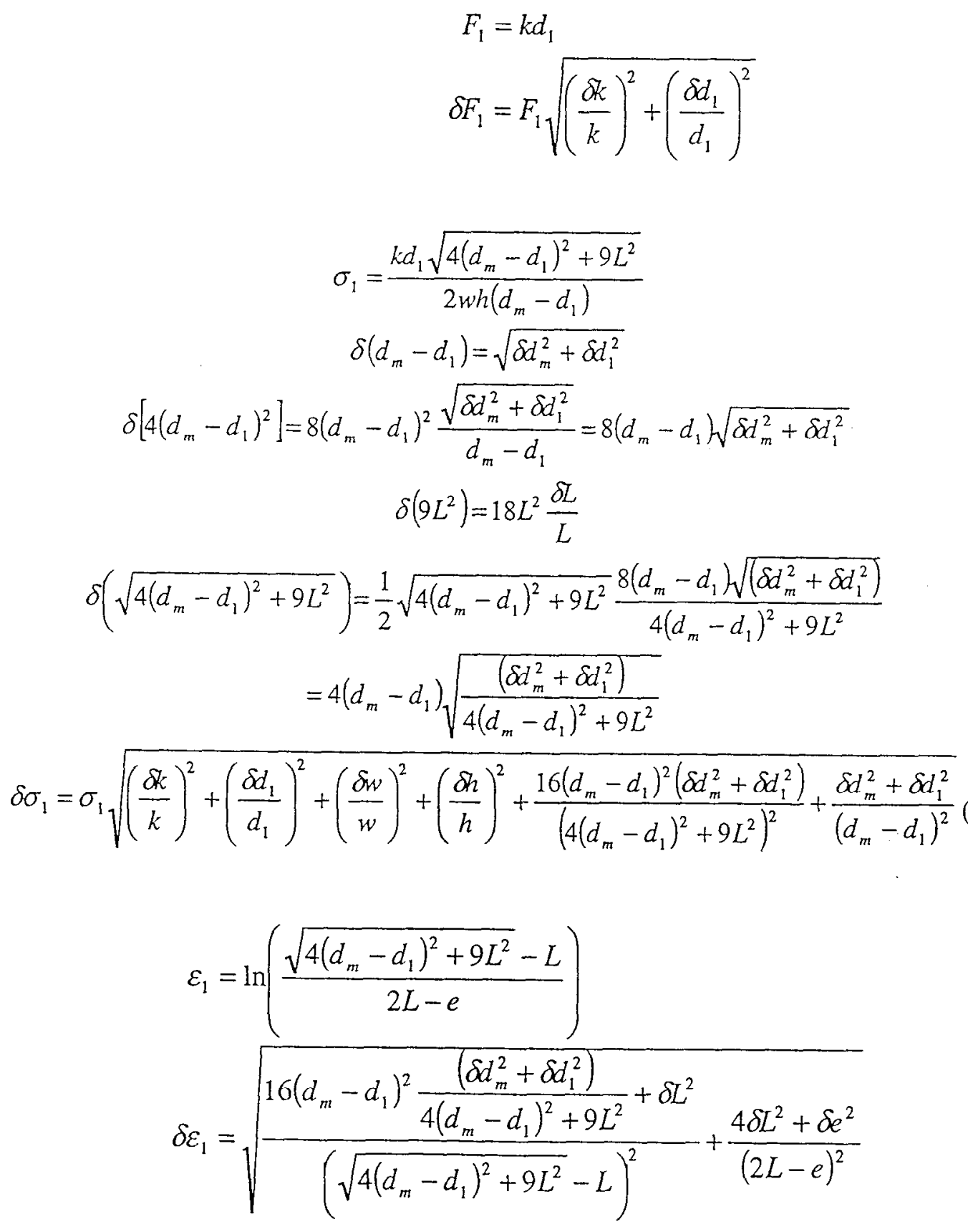

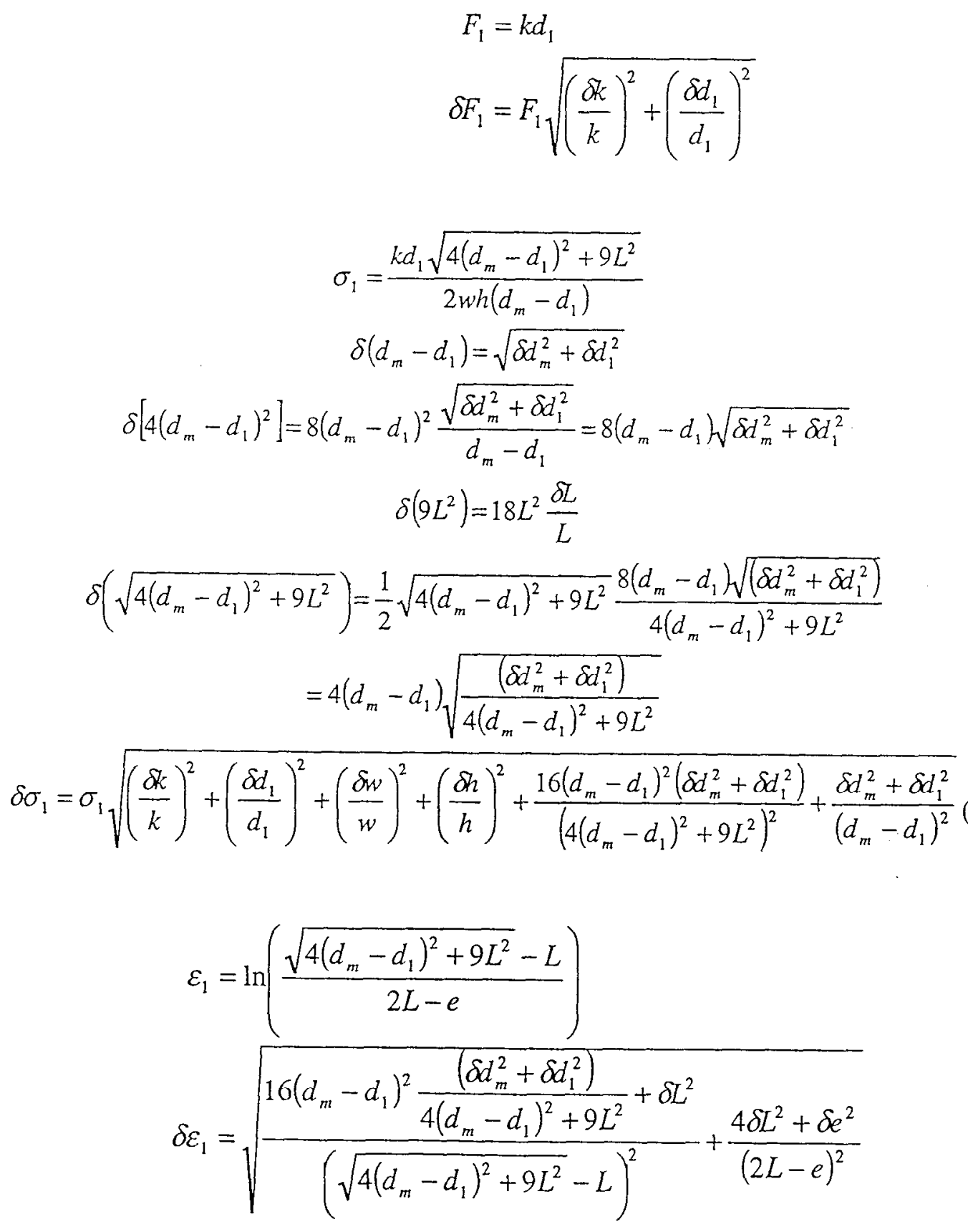




$$
\begin{aligned}
& W=\frac{k}{2}\left(d_{2}^{2}-d_{1}^{2}\right) \\
& \delta W=\frac{W}{2} \sqrt{\left(\frac{\delta k}{k}\right)^{2}+\frac{4\left(d_{2}^{2}\left(\delta d_{2}\right)^{2}+d_{1}^{2}\left(\delta d_{1}\right)^{2}\right)}{\left(d_{2}^{2}-d_{1}^{2}\right)^{2}}}
\end{aligned}
$$

When values are inserted into Equation A-7, the uncertainty for true strain, large propagated uncertainties appear. These spring from the fact that the error in the length of a ligament leg overcomes the value of $\ln \left(L^{\prime} / L\right)$ used for the strain calculation. Since this ratio depends on the fact that the numerator and denominator both contain the same value $L$, the error in the length of $L$ must be set to zero to allow for an accurate determination of the error. In so doing, the calculation of propagated error is very accurate to the values found if the extreme values for $L$ (using plus or minus the uncertainty) are substituted into the equation for strain. 


\section{Appendix B: Parts list and assembly drawing for testing instrument}

Below in Table B.1 is the parts list for the entire test instrument assembly. The parts in this list are seen in the assembly drawing in Figure B.1. The aluminum base is not seen in the parts list because it was fabricated in-house and its part drawing is shown in Figure B.2.

Table B.1: Parts list for test instrument assembly

\begin{tabular}{|l|l|l|}
\hline Company & Item & Part No. \\
\hline MicroStrain & Microminiature DVRT & N/A \\
\hline $1-800-449-3878$ & Spring loading and plastic 45 conical tip & N/A \\
\hline & Threaded body & N/A \\
\hline & CBL-1 cables & N/A \\
\hline & DEMOD1 demodulator board & N/A \\
\hline & Package and power supply for DEMOD1 & N/A \\
\hline Newport & $90^{\circ}$ Angle bracket & $360-90$ \\
\hline $1-800-222-6440$ & Coarse adjustment screw* & AJS20-1 \\
\hline & Coarse adjustment stage & 436 \\
\hline & Fine adjustment stage & $460 \mathrm{~A}-\mathrm{X}$ \\
\hline MSC Industrial Supply Co. & MTI/Mitutoyo Digimatic micrometer head & $350-711-30$ \\
\hline 1-800-645-7270 & & \\
\hline Instrument Specialties & Electrical contact strips & $97-134$ \\
\hline $1-714-579-7100$ & & \\
\hline
\end{tabular}

The specifications for the DVRT, mentioned in previous sections, are seen in Table B.2 below.

Table B.2: Electrical and Mechanical Specifications for Microminiature DVRT

\begin{tabular}{|c|c|}
\hline Core diameter & $0.5 \mathrm{~mm}$ \\
\hline Outer diameter & $1.5 \mathrm{~mm}$ \\
\hline Resolution & $1.5 \mu \mathrm{m}$ \\
\hline Linear travel & $3 \mathrm{~mm}$ \\
\hline Nonlinearity (typical) & $0.3 \%$ over $50 \%$ of linear travel \\
\hline Sensitivity & 2 volts/mm typical \\
\hline Frequency response & $7 \mathrm{kHz}$ (unfiltered) \\
\hline Temperature coefficient offset & $.0029 \% /{ }^{\circ} \mathrm{C}$ \\
\hline Temperature coefficient span & $.030 \% / \mathrm{C}$ \\
\hline Hysteresis & $+/-1 \mu \mathrm{m}$ \\
\hline Repeatability & $+1-1 \mu \mathrm{m}$ \\
\hline Polymer tip & Conical shape \\
\hline Spring loaded, spring rate & $1.3 \mathrm{lb} / \mathrm{in}(.228 \mathrm{~N} / \mathrm{mm})$ \\
& custom springs available \\
\hline $4-40$ UNC threaded body & \\
\hline
\end{tabular}

